\title{
THE SPITZER INFRARED SPECTROGRAPH SURVEY OF T TAURI STARS IN TAURUS
}

\author{
E. Furlan ${ }^{1,11}$, K. L. Luhman ${ }^{2,3}$, C. EsPaillat ${ }^{4,12}$, P. D’Alessio ${ }^{5}$, L. Adame ${ }^{6}$, P. Manoj ${ }^{7}$, K. H. Kim ${ }^{7}$, Dan M. Watson ${ }^{7}$, \\ W. J. Forrest ${ }^{7}$, M. K. McClure ${ }^{6}$, N. Calvet ${ }^{6}$, B. A. Sargent ${ }^{8}$, J. D. Green ${ }^{9}$, And W. J. Fischer ${ }^{10}$ \\ ${ }^{1}$ Jet Propulsion Laboratory, California Institute of Technology, Mail Stop 264-723, 4800 Oak Grove Drive, Pasadena, CA 91109, USA; Elise.Furlan@jpl.nasa.gov \\ ${ }^{2}$ Department of Astronomy and Astrophysics, The Pennsylvania State University, University Park, PA 16802, USA; kluhman@ astro.psu.edu \\ ${ }^{3}$ Center for Exoplanets and Habitable Worlds, The Pennsylvania State University, University Park, PA 16802, USA \\ ${ }^{4}$ Harvard-Smithsonian Center for Astrophysics, 60 Garden Street, MS-78, Cambridge, MA 02138, USA; cespaillat@ cfa.harvard.edu \\ ${ }^{5}$ Centro de Radioastronomía y Astrofísica, Universidad Nacional Autónoma de México, 58089 Morelia, Michoacán, Mexico; p.dalessio@crya.unam.mx \\ ${ }^{6}$ Department of Astronomy, The University of Michigan, 500 Church St., 830 Dennison Bldg., Ann Arbor, MI 48109, USA; adamel@ umich.edu, \\ melisma@umich.edu,ncalvet@umich.edu \\ ${ }^{7}$ Department of Physics and Astronomy, University of Rochester, Rochester, NY 14627, USA; manoj@ pas.rochester.edu, khkim@pas.rochester.edu, \\ dmw@pas.rochester.edu, forrest@pas.rochester.edu \\ ${ }^{8}$ Space Telescope Science Institute, 3700 San Martin Drive, Baltimore, MD 21218, USA; sargent@ stsci.edu \\ ${ }^{9}$ Department of Astronomy, The University of Texas at Austin, Austin, TX 78712, USA; joel@ astro.as.utexas.edu \\ ${ }^{10}$ Department of Physics and Astronomy, University of Toledo, 2801 West Bancroft Street, Toledo, OH 43606, USA; wfische@ utnet.utoledo.edu \\ Received 2011 January 25; accepted 2011 April 25; published 2011 June 22
}

\begin{abstract}
We present 161 Spitzer Infrared Spectrograph (IRS) spectra of T Tauri stars and young brown dwarfs in the Taurus star-forming region. All of the targets were selected based on their infrared excess and are therefore surrounded by protoplanetary disks; they form the complete sample of all available IRS spectra of T Tauri stars with infrared excesses in Taurus. We also present the IRS spectra of seven Class 0/I objects in Taurus to complete the sample of available IRS spectra of protostars in Taurus. We use spectral indices that are not significantly affected by extinction to distinguish between envelope- and disk-dominated objects. Together with data from the literature, we construct spectral energy distributions for all objects in our sample. With spectral indices derived from the IRS spectra we infer disk properties such as dust settling and the presence of inner disk holes and gaps. We find a transitional disk frequency, which is based on objects with unusually large 13-31 $\mu \mathrm{m}$ spectral indices indicative of a wall surrounding an inner disk hole, of about $3 \%$, and a frequency of about $20 \%$ for objects with unusually large $10 \mu \mathrm{m}$ features, which could indicate disk gaps. The shape and strength of the $10 \mu \mathrm{m}$ silicate emission feature suggests weaker $10 \mu \mathrm{m}$ emission and more processed dust for very low mass objects and brown dwarfs (spectral types M6-M9). These objects also display weaker infrared excess emission from their disks, but do not appear to have more settled disks than their higher-mass counterparts. We find no difference for the spectral indices and properties of the dust between single and multiple systems.
\end{abstract}

Key words: circumstellar matter - protoplanetary disks - stars: formation - stars: low-mass - stars: pre-main sequence

Online-only material: color figure, Supplemental data file (tar.gz)

\section{INTRODUCTION}

The young, nearby star-forming region of Taurus-Auriga constitutes one of the best sites to study star and planet formation (Kenyon et al. 2008). It is an area where mostly low-mass stars form in relative isolation, unaffected by ionizing radiation of higher-mass stars. At an age of 1-2 Myr, a substantial fraction of the young stars in Taurus-Auriga is still surrounded by circumstellar material. We can observe the entire range of premain-sequence evolutionary stages, from protostars embedded within infalling envelopes (Class 0, I objects) to T Tauri stars that are encircled by circumstellar disks (Class II objects) and those that have already dissipated their accretion disks (Class III objects). The latter two groups of objects are also divided into classical T Tauri stars (CTTS) and weak-lined T Tauri stars (WTTS) based on the strength and profile of their $\mathrm{H} \alpha$ emission line, which either reveals an accretion flow or chromospheric activity (e.g., Bertout 1989; Hartmann et al. 1994). The relatively low extinction toward Taurus-Auriga and its small distance ( $\sim 140$ pc; Bertout et al. 1999) enable sensitive, high-resolution studies across the entire electromagnetic spectrum.

\footnotetext{
${ }^{11}$ Spitzer Fellow.

${ }^{12}$ NSF Astronomy \& Astrophysics Postdoctoral Fellow.
}

Since circumstellar dust emits prominently at infrared wavelengths, it is an ideal wavelength region to study disk evolution and dissipation. The characteristics of the excess emission can be used to infer the properties and distribution of the dust grains in the disk. Models predict grain growth and settling (e.g., Miyake \& Nakagawa 1995; Weidenschilling 1997; D’Alessio et al. 2006; Ciesla 2007; Brauer et al. 2008; Birnstiel et al. 2011), the formation of planets via core accretion or gravitational instability (e.g., Pollack et al. 1996; Boss 2000; Movshovitz et al. 2010; Meru \& Bate 2011), gravitational interaction of forming, larger bodies with the disk (e.g., Takeuchi et al. 1996; Chambers 2006; Ida \& Lin 2010), and eventual dissipation of the disk via photoevaporation (e.g., Clarke et al. 2001; Alexander et al. 2006; Alexander \& Armitage 2007; Owen et al. 2010; Ercolano \& Owen 2010). Observationally, grain growth, processing, and settling have been confirmed (e.g., Meeus et al. 2003; Apai et al. 2005; Furlan et al. 2005, 2006; Kessler-Silacci et al. 2006; Sargent et al. 2006; Sicilia-Aguilar et al. 2007; Bouwman et al. 2008; Sargent et al. 2009; Furlan et al. 2009b; Olofsson et al. 2009), and infrared data also revealed disks with inner gaps or holes (Strom et al. 1989; Skrutskie et al. 1990; D'Alessio et al. 2005; Calvet et al. 2005; Brown et al. 2007, 2008, 2009; Espaillat et al. 2007a, 2007b, 2008, 2010; Kim et al. 2009; Muzerolle et al. 2010; Merín et al. 2010). These 
latter objects are often referred to as transitional disks, believed to be in transition from being optically thick to optically thin. In this work we define as transitional disks objects with low excess emission shortward of about $8 \mu \mathrm{m}$, but steeply rising spectral energy distributions (SEDs) beyond $12 \mu \mathrm{m}$, indicative of dust-depleted, optically thin inner regions surrounded by an optically thick outer disk. Following Espaillat et al. (2008), we use the term pre-transitional disks for objects with gaps (which can contain some optically thin dust) in their optically thick disks; they could be precursors of transitional disks.

Sensitive data from the Infrared Spectrograph ${ }^{13}$ (IRS; Houck et al. 2004) on board the Spitzer Space Telescope (Werner et al. 2004) allow detailed studies of the dust emission from protoplanetary disks surrounding low-mass stars and even brown dwarfs. The dust probed by these mid-infrared spectra mainly resides in the upper, warm layers of the inner disk ( 0.1-10 AU); its emission consists of optically thin emission features from the hot surface layers and continuum radiation from the cooler regions below (e.g., D'Alessio et al. 2006). Therefore, the dust features probe dust grains in the disk surface, which are not necessarily representative for the dust in the whole disk. Since collisional growth, fragmentation, turbulence, radial, and vertical mixing play a role in the observed dust distribution (Dullemond \& Dominik 2008; Johansen et al. 2008; Turner et al. 2010; Birnstiel et al. 2011), the dust properties we measure likely represent an average for a certain region of the disk. The midinfrared continuum emission probes the vertical distribution of the dust and can reveal the presence of radial structure in the disk (e.g., Dullemond et al. 2001, 2002; D'Alessio et al. 2005, 2006).

As part of a Spitzer guaranteed-time observing program, we obtained several hundred 5-38 $\mu \mathrm{m}$ IRS spectra of young stellar objects (YSOs) in nearby $(<500 \mathrm{pc})$ star-forming regions. We presented representative sets of spectra of YSOs from the Taurus star-forming in Furlan et al. (2006, 2008); here we add substantially to the sample of IRS spectra of T Tauri stars and protostars in Taurus by introducing the spectra of 81 objects obtained in later Spitzer observing campaigns as part of guaranteed- and general-observer programs. In particular, we add dozens of spectra of very low mass stars and brown dwarfs. This allows us to compare the properties of young protoplanetary disks, such as the degree of dust settling and grain processing, among low-mass stars and brown dwarfs.

This paper is structured as follows: in Section 2 we explain our observations and data reduction; in Section 3 we introduce our sample, classify the objects into evolutionary stages, and describe our adopted extinction corrections; in Section 4 we present the SEDs of our targets; in Section 5 we use spectral indices to derive disk structure; in Section 6 we analyze properties of the dust based on the $10 \mu \mathrm{m}$ silicate emission feature; in Section 7 we study the median IRS spectra for the $\mathrm{T}$ Tauri stars in our sample; in Section 8 we discuss our results; and in Section 9 we give our conclusions.

\section{OBSERVATIONS AND DATA REDUCTION}

Over the course of Spitzer's cold mission, most of the members of Taurus with infrared excesses, including the brown dwarfs, were observed with IRS. The objects presented here

\footnotetext{
13 The IRS was a collaborative venture between Cornell University and Ball Aerospace Corporation funded by NASA through the Jet Propulsion Laboratory and the Ames Research Center.
}

were observed under Spitzer guaranteed-time observing programs 2, 30540, 40145, 40302, and 50053 (PI: Houck), and under programs 179 (PI: Evans), 2300 (PI: Carr), 3303 (PI: Whittet), 3499 (PI: Apai), 20604 (PI: Boogert), 30765 (PI: Stapelfeldt), 50799 (PI: Herczeg), and 50807 (PI: Baldovin). Except for the data from programs 50053, 50799, and 50807, which were executed during IRS campaign 60 in 2009 April, the other objects were observed during different times of the Spitzer cold mission, from 2004 February to 2008 March (see Table 1 for the IRS campaign numbers and program identifiers for each object).

Some objects were re-observed at different times by different programs, in some cases to explore variability of the targets. If the same target was observed under both a guaranteedtime and general-observer program, we present only the former observation here. In our guaranteed-time programs we also reobserved a few objects that were mispointed in our original observations or had noisy spectra (CW Tau, CX Tau, CZ Tau, DM Tau, FN Tau, FO Tau, V773 Tau, ZZ Tau; Furlan et al. 2006).

All targets from guaranteed-time programs carried out after IRS campaign 12 were observed in staring mode with the two low-resolution IRS modules, Short-Low (SL) and LongLow (LL; 5.2-14 $\mu \mathrm{m}$ and $14-38 \mu \mathrm{m}$, respectively; $\lambda / \Delta \lambda \sim 90$ ). In earlier IRS campaigns, when data for guaranteed-time program 2 were taken, mapping mode was used on most targets, with two steps in the spatial direction and three steps in the dispersion direction (see Furlan et al. 2006 for details). Also, these early observations used the SL module and the high-resolution modules, Short-High (SH) and Long-High (LH; 10-19 $\mu \mathrm{m}$ and 19-37 $\mu \mathrm{m}$, respectively; $\lambda / \Delta \lambda \sim 600$ ) for targets expected to be bright ( $~$ a few Jy). The other programs with targets in the Taurus star-forming region used the staring mode, but in some cases instead of using SL and LL, they used SH and LH, or different combinations of modules. The spectra were extracted using the IDL-based SMART package (Higdon et al. 2004), starting from the Spitzer Science Center's basic calibrated data (BCD) products pipeline version S13.2 or S18.7. A detailed description of our data reduction steps can be found in Furlan et al. (2006); we followed those procedures for almost all of our targets.

A few objects that were faint or consisted of multiple components that entered the IRS slits were extracted using the optimal extraction tool in SMART (Lebouteiller et al. 2010) with the default calibration, which uses a relative spectral response function derived from three calibrator stars. For faint objects, optimal extraction resulted in improved signal-to-noise, while for multiple systems it allowed the extraction of the component at the requested position in the slit. In addition, for many faint sources fixing bad pixels defined by the bad and rogue pixel masks was not sufficient to correct all misbehaving pixels. Thus, after inspecting the two-dimensional frames by eye, additional pixels had to be added to the rogue pixel masks and then fixed before extraction of the spectrum in order to yield a spectrum free of artifacts.

Despite the different observing modes for several of our targets, the extracted and calibrated spectra are of comparable quality. We ensured that the various reduction steps, which were the same for each observing mode, resulted in a reliable spectrum for each source. For the flux calibration we used a set of proven calibrator stars and their templates (Cohen et al. 2003). Due to small mispointings, in some cases scaling factors of a few percent (at most $\sim 10 \%$ ) had to be applied to the SL 
Table 1

Members of Taurus with Infrared Excesses Observed by IRS

\begin{tabular}{|c|c|c|c|c|c|c|c|}
\hline $\begin{array}{l}\text { Name } \\
\text { (1) }\end{array}$ & $\begin{array}{c}\text { R.A. (J2000) } \\
\text { (2) }\end{array}$ & $\begin{array}{c}\text { Decl. (J2000) } \\
\text { (3) }\end{array}$ & $\begin{array}{c}\text { Camp. } \\
\text { (4) }\end{array}$ & $\begin{array}{c}\text { PID } \\
(5)\end{array}$ & $\begin{array}{c}\text { Spectral Type } \\
\text { (6) }\end{array}$ & $\begin{array}{l}A_{J} \\
(7)\end{array}$ & $\begin{array}{c}\text { Method } \\
\text { (8) }\end{array}$ \\
\hline 2MASS J04141188+2811535 & 041411.88 & +281153.5 & 29 & 2 & M6.25 & 0.71 & $J-H$ \\
\hline 2MASS J04153916+2818586 & 041539.16 & +281858.6 & 60 & 50053 & M3.75 & 0.73 & $J-K$ \\
\hline 2MASS J04155799+2746175 & 041557.99 & +274617.5 & 60 & 50807 & M5.5 & 0.54 & $J-H$ \\
\hline 2MASS J04163911+2858491 & 041639.12 & +285849.1 & 60 & 50053 & M5.5 & 0.86 & $J-H$ \\
\hline 2MASS J04201611+2821325 & 042016.11 & +282132.6 & 44 & 40302 & M6.5 & 0.24 & $J-H$ \\
\hline 2MASS J04202144+2813491 & 042021.44 & +281349.2 & 60 & 50053 & M1 & 0.0 & Edge-on \\
\hline 2MASS J04202606+2804089 & 042026.07 & +280409.0 & 44 & 40302 & M3.5 & 0.01 & $J-H$ \\
\hline 2MASS J04210795+2702204 & 042107.95 & +270220.4 & 18 & 3303 & M5.25 & 1.5 & CTTS \\
\hline 2MASS J04214631+2659296 & 042146.31 & +265929.6 & 60 & 50807 & M5.75 & 1.4 & $J-H$ \\
\hline 2MASS J04230607+2801194 & 042306.07 & +280119.5 & 39 & 30540 & M6 & 0.21 & $J-H$ \\
\hline 2MASS J04242090+2630511 & 042420.90 & +263051.2 & 35 & 30540 & M6.5 & 0.24 & $J-K$ \\
\hline 2MASS J04242646+2649503 & 042426.46 & +264950.4 & 35 & 30540 & M5.75 & 0.36 & $J-H$ \\
\hline 2MASS J04263055+2443558 & 042630.55 & +244355.9 & 60 & 50053 & M8.75 & 0.05 & $J-H$ \\
\hline 2MASS J04284263+2714039 & 042842.63 & +271403.9 & 35 & 30540 & M5.25 & 1.3 & $J-H$ \\
\hline 2MASS J04290068+2755033 & 042900.68 & +275503.4 & 39 & 30540 & M8.25 & 0.06 & $J-H$ \\
\hline 2MASS J04295950+2433078 & 042959.51 & +243307.8 & 35 & 30540 & M5 & 1.6 & $J-H$ \\
\hline 2MASS J04322415+2251083 & 043224.15 & +225108.3 & 60 & 50807 & M4.5 & 0.38 & $J-K$ \\
\hline 2MASS J04330945+2246487 & 043309.46 & +224648.7 & 60 & 50053 & M6 & 1.2 & $J-H$ \\
\hline 2MASS J04333905+2227207 & 043339.05 & +222720.7 & 60 & 50807 & M1.75 & 0.0 & Edge-on \\
\hline 2MASS J04334465+2615005 & 043344.65 & +261500.5 & 60 & 50053 & M4.75 & 1.8 & $J-H$ \\
\hline 2MASS J04335245+2612548 & 043352.46 & +261254.9 & 44 & 30540 & M8.5 & 0.90 & SpeX \\
\hline 2MASS J04361030+2159364 & 043610.31 & +215936.5 & 60 & 50053 & M8.5 & 0.05 & $J-K$ \\
\hline 2MASS J04381486+2611399 & 043814.87 & +261139.7 & 44 & 40302 & M7.25 & 0.0 & Edge-on \\
\hline 2MASS J04390163+2336029 & 043901.63 & +233602.9 & 60 & 50799 & M6 & 0.50 & $J-H$ \\
\hline 2MASS J04390396+2544264 & 043903.96 & +254426.4 & 44 & 30540 & M7.25 & 0.49 & $J-K$ \\
\hline 2MASS J04390525+2337450 & 043905.25 & +233745.0 & 60 & 50807 & K5 & 0.34 & SpeX \\
\hline 2MASS J04393364+2359212 & 043933.64 & +235921.2 & 39 & 30540 & M5 & 0.53 & $J-H$ \\
\hline 2MASS J04400067+2358211 & 044000.68 & +235821.2 & 35 & 30540 & M6 & 0.03 & $J-H$ \\
\hline 2MASS J04414489+2301513 & 044144.90 & +230151.4 & 60 & 50053 & M8.5 & 0.01 & $J-H$ \\
\hline 2MASS J04414825+2534304 & 044148.25 & +253430.5 & 19 & 2 & M7.75 & 0.78 & $J-H$ \\
\hline 2MASS J04442713+2512164 & 044427.13 & +251216.4 & 19 & 2 & M7.25 & 0.62 & $J-H$ \\
\hline 2MASS J04554535+3019389 & 045545.35 & +301938.9 & 39 & 30540 & M4.75 & 0.19 & $J-H$ \\
\hline 2MASS J04554801+3028050 & 045548.01 & +302805.0 & 39 & 30540 & M5.6 & 0.09 & $J-H$ \\
\hline AA Tau & 043455.42 & +242853.2 & 4 & 2 & K7 & 0.55 & $J-H$ \\
\hline AB Aur & 045545.83 & +303304.4 & 4 & 2 & A0 & 0.07 & (1) \\
\hline BP Tau & 041915.84 & +290626.9 & 4 & 2 & K7 & 0.3 & SpeX \\
\hline CFHT 4 & 043947.48 & +260140.7 & 19 & 3499 & M7 & 1.5 & $J-H$ \\
\hline CIDA 1 & 041417.61 & +280609.7 & 34 & 30540 & M5.5 & 1.5 & $J-H$ \\
\hline CIDA 7 & 044221.02 & +252034.4 & 35 & 30540 & M4.75 & 0.64 & $J-H$ \\
\hline CIDA 9 & 050522.86 & +253131.2 & 60 & 50053 & K7 & 0.0 & CTTS \\
\hline CIDA 11 & 050623.33 & +243220.0 & 60 & 50053 & M3.5 & 0.14 & $J-H$ \\
\hline CIDA 12 & 050754.97 & +250015.6 & 60 & 50053 & M4 & 0.21 & $J-H$ \\
\hline CIDA 14 & 044320.23 & +294006.1 & 29 & 2 & M5 & 0.24 & $J-K$ \\
\hline CI Tau & 043352.00 & +225030.2 & 4 & 2 & $\mathrm{~K} 7$ & 0.65 & SpeX \\
\hline $\mathrm{CoKu} \mathrm{Tau} / 3$ & 043540.94 & +241108.8 & 4 & 2 & M1 & 2.2 & $J-H$ \\
\hline $\mathrm{CoKu} \mathrm{Tau} / 4$ & 044116.81 & +284000.1 & 3 & 2 & M1.5 & 1.0 & $J-H$ \\
\hline CW Tau & 041417.00 & +281057.8 & 60 & 50053 & K3 & 0.56 & SpeX \\
\hline CX Tau & 041447.86 & +264811.0 & 60 & 50053 & M2.5 & 0.27 & $J-K$ \\
\hline CY Tau & 041733.73 & +282046.9 & 3 & 2 & M1.5 & 0.41 & $J-H$ \\
\hline CZ Tau & 041831.59 & +281658.5 & 60 & 50053 & M3 & 0.16 & $J-H$ \\
\hline DD Tau & 041831.13 & +281629.0 & 4 & 2 & M3.5 & 1.0 & SpeX \\
\hline DE Tau & 042155.64 & +275506.1 & 4 & 2 & M1.5 & 0.25 & SpeX \\
\hline DF Tau & 042702.80 & +254222.3 & 3 & 2 & M2 & 0.55 & $J-H$ \\
\hline DG Tau & 042704.70 & +260616.3 & 4 & 2 & K6 & 0.82 & $J-H$ \\
\hline DH Tau & 042941.56 & +263258.3 & 4 & 2 & M1 & 0.61 & $J-H$ \\
\hline DK Tau & 043044.25 & +260124.5 & 4 & 2 & $\mathrm{~K} 7$ & 0.74 & $J-H$ \\
\hline DL Tau & 043339.06 & +252038.2 & 4 & 2 & K7 & 0.56 & SpeX \\
\hline DM Tau & 043348.72 & +181010.0 & 29 & 2 & M1 & 0.0 & $J-H$ \\
\hline DN Tau & 043527.37 & +241458.9 & 4 & 2 & M0 & 0.26 & $J-H$ \\
\hline DO Tau & 043828.58 & +261049.4 & 4 & 2 & M0 & 0.85 & SpeX \\
\hline DP Tau & 044237.70 & +251537.5 & 3 & 2 & M0.5 & 0.7 & SpeX \\
\hline DQ Tau & 044653.05 & +170000.2 & 3 & 2 & M0 & 0.71 & $J-H$ \\
\hline DR Tau & 044706.21 & +165842.8 & 3 & 2 & K5 & 0.4 & SpeX \\
\hline DS Tau & 044748.59 & +292511.2 & 4 & 2 & K5 & 0.45 & SpeX \\
\hline FM Tau & 041413.58 & +281249.2 & 3 & 2 & M0 & 0.21 & CTTS \\
\hline
\end{tabular}


Table 1

(Continued)

\begin{tabular}{|c|c|c|c|c|c|c|c|}
\hline $\begin{array}{l}\text { Name } \\
(1)\end{array}$ & $\begin{array}{l}\text { R.A. (J2000) } \\
\text { (2) }\end{array}$ & $\begin{array}{c}\text { Decl. (J2000) } \\
\text { (3) }\end{array}$ & $\begin{array}{l}\text { Camp. } \\
\text { (4) }\end{array}$ & $\begin{array}{l}\text { PID } \\
(5)\end{array}$ & $\begin{array}{c}\text { Spectral Type } \\
\text { (6) }\end{array}$ & $\begin{array}{l}A_{J} \\
(7)\end{array}$ & $\begin{array}{l}\text { Method } \\
\text { (8) }\end{array}$ \\
\hline FN Tau & 041414.59 & +282758.1 & 60 & 50053 & M3 & 0.32 & $J-H$ \\
\hline FO Tau & 041449.29 & +281230.6 & 60 & 50053 & M3.5 & 0.82 & SpeX \\
\hline FP Tau & 041447.31 & +264626.4 & 3 & 2 & M4 & 0.24 & $J-H$ \\
\hline FQ Tau & 041912.81 & +282933.1 & 12 & 2 & M3 & 0.29 & $J-H$ \\
\hline FR Tau & 041935.46 & +282721.8 & 60 & 50053 & M5.25 & 0.06 & $J-H$ \\
\hline FS Tau & 042202.18 & +265730.5 & 4 & 2 & M0 & 1.2 & CTTS \\
\hline FT Tau & 042339.19 & +245614.1 & 4 & 2 & M3 & 1.0 & $J-H$ \\
\hline FV Tau & 042653.53 & +260654.4 & 4 & 2 & K5 & 1.9 & SpeX \\
\hline FV Tau/c & 042654.41 & +260651.1 & 60 & 50053 & M2.5 & 1.6 & $J-H$ \\
\hline FX Tau & 043029.61 & +242645.0 & 4 & 2 & M1 & 0.74 & $J-H$ \\
\hline FY Tau & 043230.58 & +241957.3 & 60 & 50053 & K6 & 1.7 & $J-H$ \\
\hline FZ Tau & 043231.76 & +242003.0 & 4 & 2 & M0 & 1.4 & CTTS \\
\hline GG Tau & 043230.35 & +173140.6 & 3 & 2 & K7 & 0.47 & $J-H$ \\
\hline GH Tau & 043306.22 & +240934.0 & 4 & 2 & M2 & 0.31 & SpeX \\
\hline GI Tau & 043334.06 & +242117.0 & 4 & 2 & K7 & 0.63 & $J-H$ \\
\hline [GKH94] 41 & 041946.57 & +271255.2 & 60 & 50807 & M7.5 & 7.6 & SpeX \\
\hline GK Tau & 043334.56 & +242105.8 & 12 & 2 & K7 & 0.45 & SpeX \\
\hline GM Aur & 045510.98 & +302159.5 & 4 & 2 & K7 & 0.16 & $J-H$ \\
\hline GM Tau & 043821.34 & +260913.7 & 19 & 2 & M6 & 1.4 & SpeX \\
\hline GN Tau & 043920.91 & +254502.1 & 4 & 2 & M2.5 & 1.1 & CTTS \\
\hline GO Tau & 044303.09 & +252018.8 & 3 & 2 & M0 & 0.63 & $J-H$ \\
\hline Haro 6-5B & 042200.69 & +265732.5 & 35 & 30765 & K5 & 0.0 & Edge-on \\
\hline Haro 6-13 & 043215.41 & +242859.7 & 3 & 2 & M0 & 3.2 & $J-H$ \\
\hline Haro 6-28 & 043556.84 & +225436.0 & 60 & 50053 & M3 & 1.1 & $J-H$ \\
\hline Haro 6-37 & 044658.98 & +170238.2 & 3 & 2 & K7 & 1.5 & $J-H$ \\
\hline HH 30 & 043137.47 & +181224.5 & 3 & 2 & M0 & 0.0 & Edge-on \\
\hline HK Tau & 043150.57 & +242418.1 & 4 & 2 & M0.5 & 1.1 & SpeX \\
\hline HN Tau & 043339.35 & +175152.4 & 3 & 2 & K5 & 0.3 & CTTS \\
\hline HO Tau & 043520.20 & +223214.6 & 4 & 2 & M0.5 & 0.34 & SpeX \\
\hline HP Tau & 043552.78 & +225423.1 & 4 & 2 & K3 & 1.2 & SpeX \\
\hline HQ Tau & 043547.34 & +225021.7 & 4 & 2 & K2 & 1.2 & $J-H$ \\
\hline IC 2087 IR & 043955.75 & +254502.0 & 4 & 2 & $\mathrm{~K}$ & 4.1 & CTTS \\
\hline IRAS $04108+2910$ & 041357.38 & +291819.3 & 4 & 2 & M3 & 0.4 & Opt. spec. \\
\hline IRAS 04125+2902 & 041542.78 & +290959.7 & 60 & 50807 & M1.25 & 0.75 & $J-K$ \\
\hline IRAS $04154+2823$ & 041832.03 & +283115.4 & 4 & 2 & M2.5 & 4.2 & CTTS \\
\hline IRAS $04173+2812$ & 042025.84 & +281923.6 & 60 & 50053 & M4 & 1.5 & CTTS \\
\hline IRAS 04187+1927 & 042143.24 & +193413.3 & 3 & 2 & M0 & 2.0 & $J-H$ \\
\hline IRAS F04192+2647 & 042216.76 & +265457.1 & 3 & 2 & M1.5 & 1.6 & CTTS \\
\hline IRAS 04196+2638 & 042247.87 & +264553.0 & 60 & 50053 & M1 & 1.3 & Opt. spec. \\
\hline IRAS $04200+2759$ & 042307.77 & +280557.3 & 4 & 2 & M2 & 2.2 & SpeX \\
\hline IRAS $04216+2603$ & 042444.58 & +261014.1 & 4 & 2 & M2.5 & 0.92 & $J-H$ \\
\hline IRAS $04260+2642$ & 042904.98 & +264907.3 & 35 & 30765 & K5.5 & 0.0 & Edge-on \\
\hline IRAS $04263+2654$ & 042921.65 & +270125.9 & 3 & 2 & M5.25 & 2.0 & $J-H$ \\
\hline IRAS $04278+2253$ & 043050.28 & +230008.8 & 4 & 2 & G8 & 3.6 & $J-H$ \\
\hline IRAS $04303+2240$ & 043319.07 & +224634.2 & 4 & 2 & M0.5 & 3.1 & $J-H$ \\
\hline IRAS $04370+2559$ & 044008.00 & +260525.4 & 4 & 2 & $\ldots$ & 4.2 & SpeX \\
\hline IRAS $04385+2550$ & 044138.82 & +255626.8 & 3 & 2 & M0 & 2.5 & SpeX \\
\hline IP Tau & 042457.08 & +271156.5 & 3 & 2 & M0 & 0.48 & SpeX \\
\hline IQ Tau & 042951.56 & +260644.9 & 4 & 2 & M0.5 & 0.42 & CTTS \\
\hline IS Tau & 043336.79 & +260949.2 & 4 & 2 & M0 & 0.87 & $J-H$ \\
\hline IT Tau & 043354.70 & +261327.5 & 4 & 2 & K2 & 2.2 & $J-H$ \\
\hline ITG 1 & 043756.70 & +254622.9 & 60 & 50053 & $\ldots$ & 0.1 & CTTS \\
\hline ITG 15 & 043944.88 & +260152.8 & 35 & 30540 & M5 & 1.4 & $J-K$ \\
\hline ITG 33A & 044108.26 & +255607.5 & 60 & 50053 & M3 & 1.8 & CTTS \\
\hline ITG 34 & 044110.79 & +255511.7 & 44 & 30540 & M5.5 & 1.4 & $J-H$ \\
\hline ITG 40 & 044124.64 & +254353.0 & 60 & 50053 & M3.5 & 6.7 & $J-H$ \\
\hline JH 112 A & 043249.11 & +225302.8 & 4 & 2 & K6 & 0.92 & CTTS \\
\hline JH 112 B & 043249.38 & +225308.2 & 4 & 2 & M4.25 & 1.7 & $J-K$ \\
\hline JH 223 & 044049.51 & +255119.2 & 60 & 50053 & M2 & 0.34 & $J-H$ \\
\hline KPNO 3 & 042629.38 & +262414.2 & 25 & 2 & M6 & 0.62 & $J-K$ \\
\hline KPNO 6 & 043007.25 & +260820.7 & 29 & 2 & M8.5 & 0.22 & $J-K$ \\
\hline KPNO 7 & 043057.21 & +255640.0 & 25 & 2 & M8.25 & 0.06 & $J-H$ \\
\hline KPNO 10 & 041749.55 & +281331.9 & 35 & 30540 & M5 & 0.43 & $J-K$ \\
\hline KPNO 12 & 041901.26 & +280248.7 & 29 & 2 & M9 & 0.39 & SpeX \\
\hline KPNO 13 & 042657.33 & +260628.4 & 34 & 30540 & M5 & 1.4 & $J-H$ \\
\hline
\end{tabular}


Table 1

(Continued)

\begin{tabular}{|c|c|c|c|c|c|c|c|}
\hline $\begin{array}{l}\text { Name } \\
\text { (1) }\end{array}$ & $\begin{array}{c}\text { R.A. (J2000) } \\
\text { (2) }\end{array}$ & $\begin{array}{c}\text { Decl. (J2000) } \\
\text { (3) }\end{array}$ & $\begin{array}{l}\text { Camp. } \\
\text { (4) }\end{array}$ & $\begin{array}{l}\text { PID } \\
(5)\end{array}$ & $\begin{array}{c}\text { Spectral Type } \\
\text { (6) }\end{array}$ & $\begin{array}{l}A_{J} \\
(7)\end{array}$ & $\begin{array}{l}\text { Method } \\
\text { (8) }\end{array}$ \\
\hline $\mathrm{LkCa} 15$ & 043917.80 & +222103.5 & 3 & 2 & K5 & 0.3 & SpeX \\
\hline LkCa 19 & 045536.95 & +301755.3 & 19 & 2300 & K0 & 0.37 & $J-H$ \\
\hline LkHa 358 & 043136.13 & +181343.3 & 3 & 2 & K8 & 2.4 & CTTS \\
\hline LR 1 & 041841.33 & +282725.0 & 60 & 50053 & K7 & 6.9 & $J-H$ \\
\hline MHO 3 & 041430.55 & +280514.7 & 3 & 2 & K7 & 2.1 & (2) \\
\hline MHO 5 & 043216.07 & +181246.5 & 39 & 30540 & M6 & 0.22 & $J-K$ \\
\hline MHO 6 & 043222.11 & +182742.6 & 39 & 30540 & M4.75 & 0.3 & $J-H$ \\
\hline RW Aur & 050749.54 & +302405.1 & 4 & 2 & K3 & 0.14 & (3) \\
\hline RX J0432.8+1735 & 043253.24 & +173533.8 & 24 & 179 & M2 & 0.18 & $J-H$ \\
\hline RY Tau & 042157.40 & +282635.5 & 4 & 2 & G1 & 0.9 & SpeX \\
\hline SU Aur & 045559.38 & +303401.6 & 4 & 2 & G1 & 0.25 & (4) \\
\hline T Tau & 042159.43 & +193206.4 & 3 & 2 & K0 & 0.5 & (3) \\
\hline UX Tau A+C & 043004.00 & +181349.4 & 3 & 2 & K5 & 0.13 & $J-H$ \\
\hline UY Aur & 045147.38 & +304713.5 & 4 & 2 & M0 & 0.75 & CTTS \\
\hline UZ Tau A & 043243.04 & +255231.1 & 4 & 2 & M1 & 0.09 & (3) \\
\hline V410 Anon 13 & 041817.11 & +282841.9 & 3 & 2 & M5.75 & 1.9 & $J-H$ \\
\hline V410 X-ray 1 & 041749.65 & +282936.3 & 3 & 2 & M4 & 1.7 & $J-H$ \\
\hline V410 X-ray 2 & 041834.44 & +283030.2 & 18 & 3303 & M0 & 6.2 & $J-H$ \\
\hline V410 X-ray 6 & 041901.11 & +281942.0 & 34 & 30540 & M5.5 & 0.91 & $J-K$ \\
\hline V710 Tau & 043157.80 & +182135.1 & 3 & 2 & M0.5 & 0.53 & (3) \\
\hline V773 Tau & 041412.92 & +281212.4 & 29 & 2 & K3 & 0.95 & $J-H$ \\
\hline V807 Tau & 043306.64 & +240955.0 & 4 & 2 & K5 & 0.47 & $J-H$ \\
\hline V819 Tau & 041926.26 & +282614.3 & 4 & 2 & K7 & 0.41 & $J-K$ \\
\hline V836 Tau & 050306.60 & +252319.7 & 4 & 2 & K7 & 0.42 & $J-H$ \\
\hline V892 Tau & 041840.62 & +281915.5 & 4 & 2 & B9 & 2.5 & $(5)$ \\
\hline V955 Tau & 044207.77 & +252311.8 & 4 & 2 & K7 & 1.1 & SpeX \\
\hline VY Tau & 043917.41 & +224753.4 & 3 & 2 & M0 & 0.03 & $J-H$ \\
\hline XEST 13-010 & 042936.07 & +243555.7 & 60 & 50053 & M3 & 1.9 & $J-H$ \\
\hline XZ Tau & 043140.07 & +181357.2 & 4 & 2 & M2 & 1.1 & SpeX \\
\hline $\mathrm{ZZ} \mathrm{Tau}$ & 043051.38 & +244222.3 & 29 & 2 & M3 & 0.32 & $J-H$ \\
\hline ZZ Tau IRS & 043051.71 & +244147.5 & 4 & 2 & M5 & 0.0 & Edge-on \\
\hline \multicolumn{8}{|c|}{ Class $0 /$ I objects (only objects included in this work) } \\
\hline 2MASS 04293209+2430597 & 042932.09 & +243059.7 & 60 & 50807 & $\ldots$ & $\ldots$ & $\ldots$ \\
\hline IRAS 04111+2800G & 041412.30 & +280837.3 & 60 & 50807 & $\ldots$ & $\ldots$ & $\ldots$ \\
\hline IRAM 04191+1522 & 042156.90 & +152945.8 & 29 & 179 & $\ldots$ & $\ldots$ & $\ldots$ \\
\hline IRAS 04191+1523 & 042200.43 & +153021.2 & 25 & 20604 & $\ldots$ & $\ldots$ & $\ldots$ \\
\hline IRAS 04301+2608 & 043314.25 & +261422.3 & 25 & 2 & M0 & $\ldots$ & $\ldots$ \\
\hline L1521F-IRS & 042838.96 & +265135.1 & 24 & 179 & $\ldots$ & $\ldots$ & $\ldots$ \\
\hline SST Tau 041831.2+282617 & 041831.25 & +282618.1 & 60 & 50807 & $\ldots$ & $\ldots$ & $\ldots$ \\
\hline
\end{tabular}

Notes. Column 1 lists the name of the object, Columns 2 and 3 list the coordinates (from the 2MASS Catalog), Column 4 lists the Spitzer campaign number, Column 5 lists the Spitzer program identifier, Column 6 lists the spectral type, Column 7 lists the extinction $A_{J}$ in magnitudes, and Column 8 lists the method of $A_{J}$ computation: $J-H$ and $J-K=$ derivation from these 2 MASS colors assuming photospheric near-infrared colors; SpeX $=$ derivation from the slope of the SpeX spectrum at $1 \mu \mathrm{m}$; CTTS $=$ derivation from $J-H$ and $H-K$ colors assuming intrinsic CTTS colors from Meyer et al. (1997); opt. spec. $=$ derivation from an optical spectrum was used; the numbers represent the following references: (1) DeWarf et al. 2003; (2) Luhman 2000; (3) White \& Ghez 2001; (4) Calvet et al. 2004; (5) Strom \& Strom 1994.

Objects for which the spectral type changed by more than one subclass from the one adopted in Furlan et al. (2006) are CX Tau (M0 to M2.5), CY Tau (K7 to M1.5), CZ Tau (M1 to M3), DE Tau (M0 to M1.5), DF Tau (M0 to M2), DG Tau (K6), DR Tau (K5), FN Tau (M5 to M3), FO Tau (M2 to M3.5), FT Tau (M3), GM Aur (K3 to K7), HQ Tau (K2), IRAS 04108+2910 (M0 to M3), IRAS F04192+2647 (M1.5), IRAS 04200+2759 (M2), IRAS 04216+2603 (M1 to M2.5), IRAS 04263+2654 (M5.25), IRAS 04303+2240 (M0.5), JH 112 A (K6), V807 Tau (K7 to K5), and V955 Tau (K5 to K7).

spectra to match flux levels at $14 \mu \mathrm{m}$; in general, spectra taken after about campaign 15 (2004 December) are less affected by mispointings. In our mapping mode observations the object was usually well centered in the map's two middle positions, so they are equivalent to the two nod positions of a staring mode observation. The more uncertain data are those LH spectra for which no background observation was obtained; their general flux level should be reliable, since they were scaled to meet SH at $19 \mu \mathrm{m}$, but their overall shape could be slightly distorted due to the spectral shape of the background emission. The background contribution in $\mathrm{SH}$ is negligible. The main cause for disparity in the signal-to-noise ratio of the various spectra are differences in integration time and brightness of the objects; in particular, a few faint objects had only short integration times. Overall, we estimate a spectrophotometric accuracy of 5\%-10\% for our spectra.

We note that due to the relatively large slit widths of the IRS (3".6 for SL, 4".7 for SH, 10".6 for LL, and 11". 1 for LH), objects with companions up to distances of several arcseconds could not be resolved even in SL. Therefore, for these objects only the combined spectrum could be obtained, and the flux ratios of the components given in Table 3 can be used to 
estimate the contribution of each component to the mid-infrared flux.

In the Appendix, we list objects for which we encountered problems in their reduction or which were observed in a nonstandard mode.

\section{THE SAMPLE}

\subsection{Sample Description}

The sample presented in this paper comprises $161 \mathrm{~T}$ Tauri stars $^{14}$ in Taurus-Auriga; the IRS spectra of 84 of them were already introduced and analyzed in Furlan et al. (2006). This earlier work contained a sample of the brighter low-mass, young stars with and without protoplanetary disks in this star-forming region (Class II and Class III objects, respectively), which were selected from the compilation of Kenyon \& Hartmann (1995) and from the IRAS Faint Source Catalogue. For Class II objects, this earlier sample was almost complete for spectral types up to M1, but included just a few objects for each spectral type from M2 to M6.

Five of the $161 \mathrm{~T}$ Tauri stars were already presented in Furlan et al. (2008), which analyzed the IRS spectra of 28 Class I objects in Taurus. Four of them, IRAS $04278+2253$, LkHa 358, IRAS 04154+2823, and IC 2087 IR, are objects that are most likely Class I/II transition objects with only very tenuous envelopes. They are included in this paper since their SEDs seem to be dominated by the disk emission. The fifth object, $\mathrm{HH} 30$, is surrounded by a flared circumstellar disk seen edge-on (Burrows et al. 1996); since its infrared excess is probably due to the disk alone (Watson \& Stapelfeldt 2004), it is part of the T Tauri star sample in this paper.

In addition to the $161 \mathrm{~T}$ Tauri stars, we also present the spectra of seven Class 0/I objects (2MASS 04293209+2430597, IRAM 04191+1522, IRAS 04111+2800G, IRAS 04191+1523, IRAS 04301+2608, SST Tau 041831.2+282617, and L1521FIRS) that were not part of the sample of protostars in Furlan et al. (2008). 2MASS 04293209+2430597 was identified as a protostar member of Taurus by Luhman et al. (2009). IRAM $04191+1522$ is a deeply embedded Class 0 protostar discovered by André et al. (1999); it is located about $1^{\prime}$ to the southwest of the Class I object IRAS 04191+1523 (Tamura et al. 1991; Moriarty-Schieven et al. 1992). IRAS 04111+2800G was discovered in reprocessed IRAS maps by Prusti et al. (1992) and found to be associated with an ammonia core. L1521F-IRS is a low-luminosity object embedded in a dense core, likely a Class 0 object (Bourke et al. 2006). IRAS 04301+2608 was identified as a pre-main-sequence candidate in Kenyon et al. (1994); we will detail in Section 3.3 how we inferred its evolutionary state. Finally, SST Tau 041831.2+281617 was identified as a candidate Taurus member by Rebull et al. (2010) based on mid-infrared photometry.

The seven Class 0/I objects presented here, and the 23 Class I objects from Furlan et al. (2008) that are embedded protostars (whose infrared and longer-wavelength emission seems dominated by an envelope), form the full sample of IRS spectra of Class 0/I objects in Taurus. According to the list of embedded sources in Taurus from Luhman et al. (2010), only two Class I objects, L1551NE and IRAS 04166+2706, do not have any IRS spectra (note that Luhman et al. 2010 pointed out that the source labeled IRAS 04166+2706 in Furlan et al. 2008 is actually IRAS 04166+2708).

\footnotetext{
14 ASCII versions of the 161 reduced IRS spectra are available in the electronic edition of the journal as a supplemental tar.gz file.
}

To summarize, here we include all IRS observations of Class II objects (i.e., T Tauri stars with infrared excesses) that lie in the Taurus-Auriga star-forming region; together with the spectra presented in Furlan et al. $(2006,2008)$ and the spectra of the seven Class 0/I objects mentioned above, they comprise close to the full sample of Taurus YSOs that are still surrounded by primordial material (see Table 1). Some Class III objects in Taurus have also been observed by IRS; they are not included in this work, but most of them are presented in Furlan et al. (2006). A few objects in our sample were recently identified in surveys of the Taurus star-forming region (Luhman et al. 2009, 2010; Rebull et al. 2010) using the Infrared Array Camera (IRAC; Fazio et al. 2004) and the Multiband Imaging Photometer for Spitzer (MIPS; Rieke et al. 2004). Based on these surveys, there are a total of nearly $200 \mathrm{~T}$ Tauri stars with at least some infrared excess in Taurus; therefore, our sample is about $80 \%$ complete.

Two objects that were part of the Class II object sample of Furlan et al. (2006), F04101+3103 and F04570+2520, turned out not to be members of Taurus (E. Mamajek 2010, private communication). Based on proper motion, the former is a member of the Per OB2 association, which is about twice as distant as Taurus (De Zeeuw et al. 1999), while the latter is a background asymptotic giant branch star.

Three of the objects that were only identified by their IRAS Faint Source Catalog number in Furlan et al. (2006) are renamed in this publication: F04147+2822 was changed to V410 X-ray 1, F04262+2654 to IRAS $04263+2654$, and F04297+2246 A to JH 112 A. We kept the name of IRAS F04192+2647, even though in the literature it is sometimes referred to as CFHT-Tau 21 (Guieu et al. 2006; Kraus \& Hillenbrand 2009; Rebull et al. 2010). This object seems to be a single star (in Furlan et al. 2006 we listed a companion at a distance of $23^{\prime \prime} .3$ ), while the recently identified companion of JH 112, which lies at a distance of 6.'6 (Kraus \& Hillenbrand 2009), is a T Tauri star with an infrared excess (Luhman et al. 2010). We attempted to obtain IRS spectra of both components of the $\mathrm{JH} 112$ system in campaign 4, but due to their close proximity the LL spectrum of JH $112 \mathrm{~B}$ is not reliable. Thus, here we include only the SL spectrum of JH 112 $\mathrm{B}$, and both the SL and LL spectrum of JH $112 \mathrm{~A}$.

\subsection{Spectral Types, T Tauri Types, and Multiplicity}

The spectral type distribution for our IRS sample of Class II objects in Taurus that have a measured spectral type is shown in Figure 1. The majority of spectral types are from Kenyon \& Hartmann (1995, and references therein), Briceño et al. (2002), Luhman (2004, 2006), and Luhman et al. (2009). Only two Class II objects in our sample, IRAS 04370+2559 and ITG 1, do not have a known spectral type. Compared to Furlan et al. (2006), we particularly expand on objects with spectral types later than M2. Since we attempted to gather the most recent, reliable spectral types from the literature, several objects have revised spectral types; changes larger than one subclass are noted in the footnote of Table 1 . High-resolution optical or nearinfrared spectra are preferable to determine spectral types, since the effect of veiling (the appearance of weaker stellar absorption lines due to excess continuum emission) can be measured more easily (e.g., Hartigan et al. 1991). Therefore, whenever available, we adopted the spectral types derived from high-resolution spectra. The typical uncertainty for our spectral types is \pm 1 subtype, but we note that the accuracy of the classification for M-type stars is often higher, while it is lower for objects with veiling. 


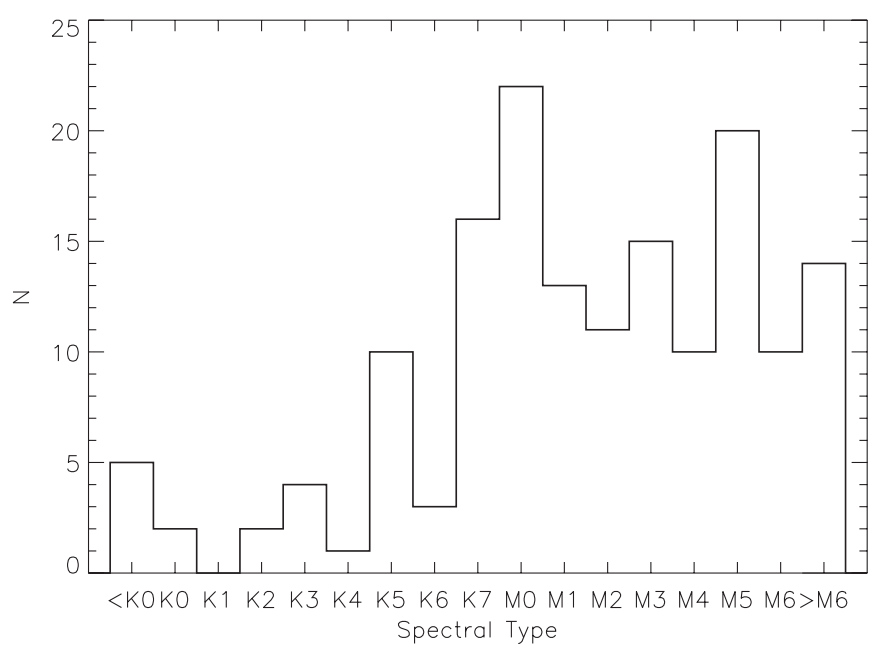

Figure 1. Spectral type distribution for all Class II objects in Taurus that have measured spectral types and were observed by IRS (see Table 1).

The median spectral type for our current, larger sample is M2, while the median for our earlier sample was M0. At the age of Taurus, the highest-mass brown dwarfs $\left(\sim 0.08 M_{\odot}\right)$ have spectral types of M6-M6.5 (e.g., Baraffe et al. 2002), so our sample contains at least 14 brown dwarfs.

Given that we selected YSOs with infrared excesses for our sample, most of our targets are expected to be CTTS and therefore surrounded by accretion disks. In fact, only somewhat more than a dozen targets are WTTS (see Table 2). We note that HQ Tau and IQ Tau would be considered WTTS based on their $\mathrm{EW}(\mathrm{H} \alpha)$ values, which amounts to $2 \AA$ for the former object and 8-25 $\AA$ for the latter one (Kenyon et al. 1998; Luhman et al. 2009; Nguyen et al. 2009). However, the $\mathrm{H} \alpha 10 \%$ width is regarded a better indicator for accretion (White \& Basri 2003), with the lowest values for accretors between $\sim 200 \mathrm{~km} \mathrm{~s}^{-1}$ and $\sim 270 \mathrm{~km} \mathrm{~s}^{-1}$, depending on the stellar mass (White \& Basri 2003; Jayawardhana et al. 2003; Natta et al. 2004). Highresolution optical spectra obtained by Nguyen et al. (2009) showed that the $\mathrm{H} \alpha 10 \%$ widths are larger than the accretion threshold for both HQ Tau and IQ Tau, and therefore they are actually CTTS. FN Tau seems to lie on the borderline between a CTTS and WTTS, with an EW(H $\alpha)$ of $16-22 \AA$ and $\mathrm{H} \alpha 10 \%$ width of $195 \mathrm{~km} \mathrm{~s}^{-1}$ (Kenyon et al. 1998; Muzerolle et al. 2003). A flare or an actual change in accretion rate has been observed in the optical for FN Tau (Guenther \& Ball 1999), so this object could be a (highly) variable CTTS.

A significant fraction of the $\mathrm{T}$ Tauri stars in our sample are members of multiple systems (there are 53 systems; see Table 3 ). However, the census of multiple systems is very likely not complete. Multiplicity surveys of the Taurus star-forming region (Simon et al. 1992, 1995; Ghez et al. 1993; Leinert et al. 1993; Richichi et al. 1994; White \& Ghez 2001; Kraus \& Hillenbrand 2009; Kraus et al. 2011) are typically carried out at $2.2 \mu \mathrm{m}$ and sensitive to companions up to $2-3$ mag fainter than the primary star at distances down to about 0 .'1; some of these surveys were able to resolve companions as close as 0 .'005 from the primary. Including binaries at wide separations $\left(\gtrsim 3^{\prime \prime}\right)$, Kraus et al. (2011) found that about $70 \%$ of objects in Taurus are members of multiple systems. Thus, our sample likely contains several objects that have as yet unidentified companions, in particular among the very low mass stars and brown dwarfs that have not yet been targeted by multiplicity
Table 2

Weak-lined T Tauri Stars With Infrared Excesses in Taurus Observed by IRS

\begin{tabular}{lccc}
\hline \hline Name & Spectral Type & EW(H $\alpha)(\AA)$ & $\begin{array}{c}\text { Ref. } \\
(1)\end{array}$ \\
\hline 2MASS J04153916+2818586 & M3.75 & $(3)$ & $(4)$ \\
2MASS J04202606+2804089 & M3.5 & 5 & 1 \\
2MASS J04214631+2659296 & M5.75 & 17.32 & 1 \\
2MASS J04242646+2649503 & M5.75 & 9.95 & 2 \\
2MASS J04284263+2714039 & M5.25 & 15 & 2 \\
2MASS J04322415+2251083 & M4.5 & 14 & 3 \\
CoKu Tau/3 & M1 & 4.6 & 1 \\
CoKu Tau/4 & M1.5 & 2.8 & 4 \\
IRAS 04125+2902 & M1.25 & 2.3 & 5 \\
JH 223 & M2 & 4.2 & 1 \\
KPNO 13 & M5 & 7.5 & 5 \\
LkCa 19 & K0 & 1 & 6 \\
RXJ 0432.8+1735 & M2 & 1.7 & 7 \\
V410 X-ray 6 & M5.5 & $9-13$ & 8 \\
V819 Tau & K7 & 3.1 & 9,10 \\
VY Tau & M0 & 7.3 & 5 \\
& & & 5
\end{tabular}

Notes. Column 1 lists the name of the object, Column 2 lists the spectral type, Column 3 lists the equivalent width of the $\mathrm{H} \alpha$ emission line, and Column 4 lists the reference for the data in Column 3.

References. (1) Luhman et al. 2009; (2) Guieu et al. 2006; (3) Luhman 2004; (4) Herbig \& Bell 1988 (5) Kenyon et al. 1998; (6) Mohanty et al. 2005; (7) White \& Ghez 2001; (8) Wichmann et al. 1996; (9) Strom \& Strom 1994; (10) Martín et al. 1999.

surveys. For the purposes of our analysis, we only consider multiple T Tauri stars within a separation range of $20^{\prime \prime}$, which corresponds to projected separations of less than $2800 \mathrm{AU}$. More widely separated companions are well resolved by all IRS modules and likely do not affect the disks of the other components; in addition, it is more difficult to assess whether a wide pair really forms a bound system.

Binaries are most common in our sample ( $76 \%$ of multiple systems), but there are a few triple and quadruple systems (11\% and $13 \%$, respectively). Twelve are known to be mixed systems, with one (or more) components CTTS and the others WTTS. A prominent example is V773 Tau, which is considered a WTTS based on its EW(H $\alpha)$ of 2-3 A (Feigelson et al. 1994; Kenyon et al. 1998), but is actually a tight quadruple system with components in apparently different stages of evolution: it consists of a spectroscopic binary (AB) whose spectral types are $\mathrm{K} 2$ and $\mathrm{K} 7$, an $\sim \mathrm{M} 0$ companion (C), and an infrared companion (D), all located within $\sim 0$ '.25 (Duchêne et al. 2003). V773 Tau AB are WTTS with no excess emission out to $3.8 \mu \mathrm{m}$, while V773 Tau C is a CTTS with a mid-infrared excess; however, the deeply embedded $\mathrm{D}$ component dominates the flux in the mid-infrared (Duchêne et al. 2003). The IRS spectrum of V773 Tau contains emission from all four components, with the contribution from V773 Tau D the strongest at $\gtrsim 10 \mu \mathrm{m}$. This demonstrates that at least for a few multiple systems, the interpretation of the infrared excess is made difficult by a complex distribution of circumstellar material, but for the majority of systems either a circumstellar or circumbinary disk dominates the mid-infrared flux (see also McCabe et al. 2006).

\subsection{Classification}

While most YSOs with excesses in Taurus have already been classified as Class I or Class II objects based on their SED 
Table 3

Multiple T Tauri Stars in Taurus with Separations $<20^{\prime \prime}$ Observed by IRS

\begin{tabular}{|c|c|c|c|c|c|}
\hline $\begin{array}{l}\text { Name } \\
\text { (1) }\end{array}$ & (2) & $\begin{array}{c}\text { Separation } \\
\text { (arcsec) } \\
(3)\end{array}$ & $\begin{array}{c}\text { TTS Type } \\
\text { (4) }\end{array}$ & Flux Ratio [Wave Band] & $\begin{array}{c}\text { References } \\
\text { (6) }\end{array}$ \\
\hline 2MASS J04284263+2714039 & 2 & 0.62 & $\mathrm{~W}$ & $2.29[K]$ & 1,2 \\
\hline 2MASS J04414489+2301513 & 4 & $0.105(\mathrm{~A}-\mathrm{B}), 12.4(\mathrm{~A}-\mathrm{C}), 0.23(\mathrm{C}, \mathrm{D})$ & $\mathrm{C}(\mathrm{A})+\mathrm{W}(\mathrm{C})$ & $4.1(\mathrm{~A}, \mathrm{~B}), 0.05(\mathrm{~A}, \mathrm{C}), 11.5(\mathrm{C}, \mathrm{D})[K]$ & 3,4 \\
\hline 2MASS J04554801+3028050 & 2 & 6.3 & $\mathrm{C}$ & $7.4[K]$ & 5,6 \\
\hline CIDA 9 & 2 & 2.33 & $\mathrm{C}$ & $1.8[K]$ & 5 \\
\hline CIDA 11 & 2 & 0.097 & $\mathrm{C}$ & $1.3[K]$ & 7,8 \\
\hline $\mathrm{CoKu} \mathrm{Tau/3}$ & 2 & 2.05 & $\mathrm{~W}$ & $10.22[N]$ & 9,10 \\
\hline $\mathrm{CoKu} \mathrm{Tau} / 4$ & 2 & 0.053 & W & $1.2[K]$ & 11 \\
\hline $\mathrm{CZ} \mathrm{Tau}^{\mathrm{c}}$ & 2 & 0.32 & $\mathrm{~W}+\mathrm{C}$ & $1.2[L]$ & 9 \\
\hline $\mathrm{DD} \mathrm{Tau}^{\mathrm{c}}$ & 2 & 0.54 & $\mathrm{C}$ & $1.75[N]$ & 9,10 \\
\hline DF Tau & 2 & 0.09 & $\mathrm{C}$ & $1.6[K]$ & 9 \\
\hline DH Tau ${ }^{\mathrm{d}}$ & 2 & 15 & $\mathrm{C}+\mathrm{W}$ & $2.0[L]$ & $12,13,14$ \\
\hline DK Tau & 2 & 2.5 & $\mathrm{C}$ & $8.53[N]$ & $12,14,10$ \\
\hline DP Tau & 2 & 0.107 & $\mathrm{C}$ & $1.4[K]$ & 7,8 \\
\hline DQ Tau & 2 & SB & $\mathrm{C}$ & $\sim 1$ & 15 \\
\hline FO Tau & 2 & 0.15 & $\mathrm{C}$ & $1.7[L]$ & 9 \\
\hline FQ Tau & 2 & 0.76 & $\mathrm{C}+\mathrm{W}$ & $1.13[N]$ & $9,16,10$ \\
\hline FS Tau ${ }^{\mathrm{e}}$ & 3 & $0.23(\mathrm{Aa}-\mathrm{Ab}), 20(\mathrm{~A}-\mathrm{B})$ & $\mathrm{C}$ & $5.5(\mathrm{Aa}, \mathrm{Ab})[L]$ & 9,16 \\
\hline FV Tau ${ }^{\mathrm{f}}$ & 4 & $12.3(\mathrm{AB}-\mathrm{CD}), 0.72(\mathrm{~A}-\mathrm{B}), 0.71(\mathrm{C}-\mathrm{D})$ & $\mathrm{C}+\mathrm{C}(\mathrm{AB}), \mathrm{W}+\mathrm{C}(\mathrm{CD})$ & $2.2(\mathrm{~A}, \mathrm{~B}), 0.74(\mathrm{C}, \mathrm{D})[N]$ & $9,16,10$ \\
\hline FX Tau & 2 & 0.89 & $\mathrm{C}+\mathrm{W}$ & $2.75[N]$ & $9,17,10$ \\
\hline FZ Tau ${ }^{g}$ & 2 & 16.9 & $\mathrm{C}$ & $2.2[L]$ & $12,13,18$ \\
\hline GG Tau & 4 & $10.3(\mathrm{~A}-\mathrm{B}), 0.24(\mathrm{Aa}-\mathrm{Ab}), 1.4(\mathrm{Ba}-\mathrm{Bb})$ & $\mathrm{C}$ & $1.03(\mathrm{Aa}, \mathrm{Ab})[N]$ & 9,10 \\
\hline $\mathrm{GH} \mathrm{Tau}^{\mathrm{h}}$ & 2 & 0.30 & $\mathrm{C}$ & $1.45[N]$ & 9,10 \\
\hline GK Tau ${ }^{\mathrm{i}}$ & 3 & $2.5(\mathrm{Aa}-\mathrm{Ab}), 12.9(\mathrm{~A}-\mathrm{B})$ & $\mathrm{C}(\mathrm{A}, \mathrm{B})$ & $44(\mathrm{Aa}, \mathrm{Ab})[\mathrm{I}], 1.4(\mathrm{~A}, \mathrm{~B})[L]$ & $17,13,18$ \\
\hline GN Tau & 2 & 0.33 & $\mathrm{C}$ & $1.2[L]$ & 9,19 \\
\hline Haro 6-28 & 2 & 0.66 & $\mathrm{C}$ & $0.94[N]$ & 9,10 \\
\hline Haro 6-37 & 3 & $2.61(\mathrm{~A}-\mathrm{B}), 0.30(\mathrm{Aa}-\mathrm{Ab})$ & $\mathrm{C}$ & $1.19(\mathrm{~A}, \mathrm{~B})[N]$ & 20,10 \\
\hline HH 30 & 2 & 0.1 & $\mathrm{C}$ & $\ldots$ & 21 \\
\hline HK Tau & 2 & 2.34 & $\mathrm{C}$ & $30[N]$ & $9,12,10$ \\
\hline HN Tau & 2 & 3.11 & $\mathrm{C}$ & $65[L]$ & 9,12 \\
\hline HP Tau ${ }^{\mathrm{j}}$ & 2 & 0.017 & $\mathrm{C}$ & $7.8[K]$ & $22,12,13$ \\
\hline IRAS $04263+2654$ & 2 & 0.22 & $\ldots$ & $2.3[K]$ & 2 \\
\hline IRAS $04278+2253$ & 2 & 6.8 & $\mathrm{C}$ & $>32[K]$ & 23 \\
\hline IRAS $04370+2559$ & 2 & 4.3 & $\mathrm{C}$ & $58[K]$ & 24,25 \\
\hline IRAS $04385+2550$ & 2 & 18.9 & $\mathrm{C}$ & $19[K]$ & 26,23 \\
\hline IS Tau & 2 & 0.22 & $\mathrm{C}+\mathrm{W}$ & $8.6[L]$ & 9 \\
\hline IT Tau & 2 & 2.39 & $\mathrm{C}$ & $2.95[N]$ & $9,17,10$ \\
\hline ITG 15 & 2 & 2.99 & $\mathrm{C}$ & $156[K]$ & 24,25 \\
\hline ITG 33 & 2 & 5.17 & $\mathrm{C}$ & $66[K]$ & 24,25 \\
\hline JH 112 & 2 & 6.6 & $\mathrm{C}$ & $2.7[L]$ & 4,18 \\
\hline JH 223 & 2 & 2.1 & $\mathrm{~W}$ & $11.7[K]$ & 5 \\
\hline RW Aur & 2 & 1.42 & $\mathrm{C}$ & $13.63[N]$ & 9,10 \\
\hline T Tau & 3 & $0.7(\mathrm{~N}-\mathrm{S}), 0.1(\mathrm{Sa}-\mathrm{Sb})$ & $\mathrm{C}$ & $0.89(\mathrm{~N}-\mathrm{S})[N]$ & $9,27,10$ \\
\hline UX Tau & 4 & $5.86(\mathrm{~A}-\mathrm{B}), 0.138(\mathrm{Ba}-\mathrm{Bb}), 2.63(\mathrm{~A}-\mathrm{C})$ & $\mathrm{C}(\mathrm{A})+\mathrm{W}(\mathrm{B}, \mathrm{C})$ & $11.9(\mathrm{~A}, \mathrm{~B}), 59.7(\mathrm{~A}, \mathrm{C})[N]$ & $9,20,10$ \\
\hline UY Aur & 2 & 0.88 & $\mathrm{C}$ & $2.07[N]$ & $9,16,10$ \\
\hline UZ Tau & 4 & $\mathrm{SB}(\mathrm{A}), 3.54(\mathrm{~A}-\mathrm{Ba}), 0.37(\mathrm{Ba}-\mathrm{Bb})$ & $\mathrm{C}$ & $8.02(\mathrm{~A}, \mathrm{~B})[N]$ & $9,16,10$ \\
\hline V710 $\mathrm{Tau}^{\mathrm{k}}$ & 2 & 3.17 & $\mathrm{C}+\mathrm{W}$ & $12.73[N]$ & $9,4,10$ \\
\hline V773 Tau ${ }^{l}$ & 4 & $\mathrm{SB}(\mathrm{AB}), 0.12(\mathrm{AB}-\mathrm{C}), 0.24(\mathrm{AB}-\mathrm{D})$ & $\mathrm{W}(\mathrm{AB})+\mathrm{C}(\mathrm{C})$ & $1.1(\mathrm{AB}, \mathrm{C}), 1.4(\mathrm{AB}, \mathrm{D})[L]$ & 9,28 \\
\hline V807 Tau ${ }^{\mathrm{h}}$ & 3 & $0.30(\mathrm{~A}-\mathrm{B}), 0.04(\mathrm{Ba}-\mathrm{Bb})$ & $\mathrm{C}+\mathrm{W}$ & $3.6(\mathrm{~A}, \mathrm{~B})[L]$ & 9,29 \\
\hline V892 Tau & 3 & $0.06(\mathrm{Aa}-\mathrm{Ab}), 4.10(\mathrm{~A}-\mathrm{B})$ & W & $4.0(\mathrm{~A}, \mathrm{~B})[K]$ & 30,31 \\
\hline V955 Tau ${ }^{\mathrm{m}}$ & 4 & $10.5(\mathrm{AB}-\mathrm{CD}), 0.33(\mathrm{~A}-\mathrm{B}), 0.23(\mathrm{C}-\mathrm{D})$ & $\mathrm{C}+\mathrm{W}(\mathrm{A}, \mathrm{B}), \mathrm{W}(\mathrm{C}, \mathrm{D})$ & $1.76(\mathrm{~A}, \mathrm{~B})[N], 1.7(\mathrm{C}, \mathrm{D})[L]$ & 9,10 \\
\hline VY Tau & 2 & 0.66 & W & $3.4[L]$ & 9 \\
\hline $\mathrm{XZ} \mathrm{Tau}{ }^{\mathrm{n}}$ & 2 & 0.30 & $\mathrm{C}$ & $0.3[L]$ & 9 \\
\hline $\mathrm{ZZ} \mathrm{Tau}^{\circ}$ & 2 & 0.06 & $\mathrm{C}$ & $1.5[K]$ & 32 \\
\hline
\end{tabular}

Notes. Column 1 lists the name of the object, Columns (2) and (3) list the number of components and their separations (SB means spectroscopic binary), Column 4 lists the T Tauri star type ("C" for CTTS and "W" for WTTS), Column 5 lists the flux ratio and, in square brackets, the wave band in which the flux ratio was measured, and Column 6 lists the references. If only one T Tauri star type is listed in Column (4), it either applies to all components of a multiple system, or the H $\alpha$ emission from the various components could not be resolved.

${ }^{a}$ Components A and B refer to the binary 2MASS source J04414489+2301513, while components C and D are the binary 2MASS J04414565+2301580.

b The other component is 2MASS J04554757+3028077.

c CZ Tau and DD Tau are separated by 30.1 and could form a very wide hierarchical binary.

${ }^{\mathrm{d}}$ The other component is DI Tau. 
e FS Tau B is also known as Haro 6-5B.

${ }^{\mathrm{f}}$ Components A and B refer to the binary FV Tau, while components C and D refer to the binary FV Tau/c.

$\mathrm{g}$ The other component is FY Tau.

${ }^{\text {h }}$ GH Tau and V807 Tau are separated by 21".5 and could form a wide binary (Kraus \& Hillenbrand 2007).

i Component A is GK Tau, component B is GI Tau.

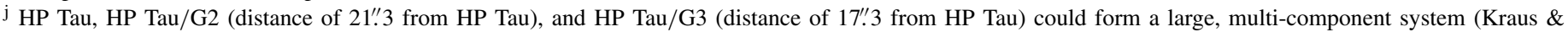
Hillenbrand 2007, 2009).

k V710 Tau could form a very wide binary system with LkHa 267, which lies at a distance of $28^{\prime \prime}$.

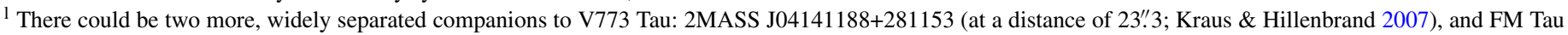
(at a distance of 37".3; Monin et al. 1998).

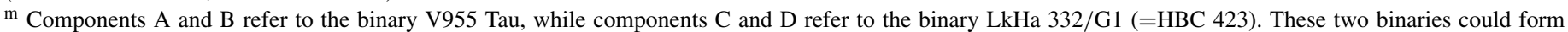

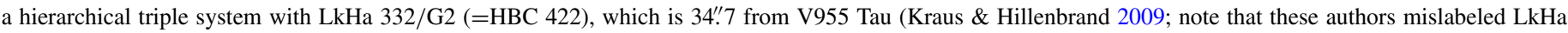
332/G1 as LkHa 332/G2 and vice versa).

${ }^{n}$ HL Tau is 23". 3 from XZ Tau; they could form a wide binary (Kraus \& Hillenbrand 2007).

' ZZ Tau could have another, very wide companion in ZZ Tau IRS, which lies at a distance of $35^{\prime \prime}$.

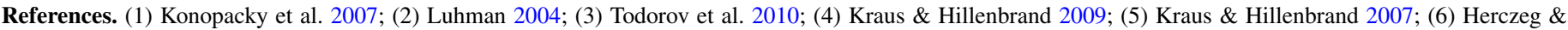

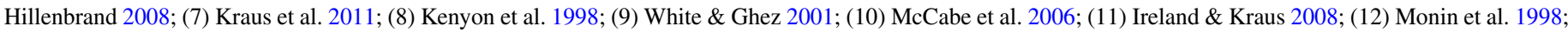

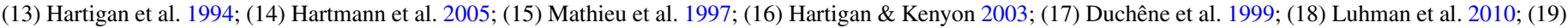

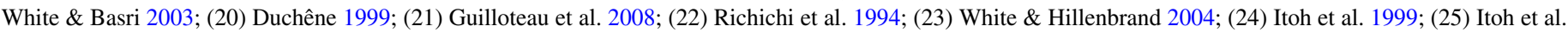

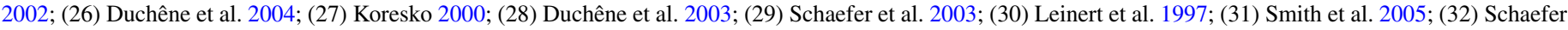
et al. 2006.

and other indicators (Kenyon \& Hartmann 1995; Luhman et al. 2010; Rebull et al. 2010), for some objects it is not clear to which degree they are still embedded in circumstellar material. In order to determine whether these objects are dominated by disk or envelope excess emission in the infrared, we constructed "extinction-free" indices following McClure et al. (2010). These indices measure the slope of the SED between 5.3 and $12.9 \mu \mathrm{m}$ $\left(n_{5-12}\right)$ and between 12.9 and $19.8 \mu \mathrm{m}\left(n_{12-20}\right)$, using the observed IRS spectra. They are defined at wavelengths for which the wavelength-dependent extinction $A_{\lambda} / A_{J}$ is the same according to the McClure (2009) extinction curve for objects in molecular clouds with $A_{J}>2.5$. For less extinguished objects, which includes the majority of our sample, somewhat different extinction curves apply (see Section 3.4), and therefore these indices are not truly extinction-free. However, the extinction corrections over the mid-infrared spectral range are smaller for the $A_{J}<2.5$ extinction curves, and for all curves they are smaller at 5, 12, and $20 \mu \mathrm{m}$ than at $2.2 \mu \mathrm{m}$. Therefore, these indices are still less dependent on foreground extinction than the standard $n_{2-25}$ index used for classification (Lada \& Wilking 1984; Lada 1987).

Figure 2 shows these "extinction-free" indices for the sample of YSOs in Taurus from Furlan et al. $(2006,2008)$ and this work for which at least the 5-20 $\mu \mathrm{m}$ IRS spectrum is available (see also Table 4). Thus, the sample for this plot consists of Class I, II, and III objects. As in McClure et al. (2010), the region with $n_{5-12}>-0.2$ is dominated by protostars surrounded by envelopes, while the region with smaller $n_{5-12}$ values is occupied by objects dominated by disks. Spectral indices smaller than about -2 reveal objects with very little circumstellar material.

Transitional disks, which are characterized by little excess $\lesssim 8 \mu \mathrm{m}$, but rising SEDs beyond $12 \mu \mathrm{m}$, can be found in the $n_{5-12}<-0.2$ and $n_{12-20}>2$ region. Here we identify two additional transitional disks: IRAS $04125+2902$ and V410 X-ray 6. They will be discussed in more detail in Section 4.5. SU Aur, whose $n_{12-20}$ value amounts to 2.2 , also lies in this region, but given its early spectral type of G1 (Calvet et al. 2004), it likely has a flared disk geometry more similar to that of a Herbig Ae/Be star (see, e.g., Meeus et al. 2001). IRAS $04302+2247$ and $04368+2557$ also lie in the transitional disk region, but they are embedded protostars seen edge-on and therefore have a steeply decreasing 5-12 $\mu \mathrm{m}$ spectral index, but a very large $n_{12-20}$ value (see Furlan et al. 2008).

Two objects have close to photospheric fluxes (spectral index of $\sim-3$ ) from 5 to $12 \mu \mathrm{m}$, but some weak excess beyond $12 \mu \mathrm{m}$ : V819 Tau and RX J0432.8+1735. In Figure 2, they lie in the debris disk region at $n_{12-20}$ values of -1.06 and 0.55 , respectively. Their SEDs will be discussed in Section 4.5.

In the envelope region, we identify a few Class I objects not studied in Furlan et al. (2008): IRAS 04301+2608, IRAS 04111+2800G, 2MASS 04293209+2430597, and SST Tau $041831.2+281617$. These objects all have rising mid-infrared SEDs, and the latter three also display a silicate absorption feature at $10 \mu \mathrm{m}$ and various ice absorption features, which suggest that they are embedded in protostellar envelopes. Thus, the IRS spectrum indicates that the candidate Taurus member SST Tau $041831.2+281617$ from Rebull et al. (2010) is a new protostellar member of this star-forming region. IRAS $04301+2608$ has a spectral type of M0 and little excess out to $3 \mu \mathrm{m}$; its SED rises steeply beyond $6 \mu \mathrm{m}$, similar to transitional disks. It was suggested to be surrounded by an edge-on disk, with optical and near-infrared emission dominated by scattered light (Briceño et al. 2002). However, the large spectral indices over the entire mid-infrared spectrum rather suggest that it is a Class I object. Its SED is indeed similar to that of CoKu Tau/1 (see Furlan et al. 2008), which is an edge-on protostellar system with only a tenuous envelope (see Figure 3).

2MASS J04202606+2804089, CZ Tau, FN Tau, Haro 65B, MHO 3, UY Aur, and V892 Tau also lie in the envelope region, but, except for Haro 6-5B, they have SEDs that are typical of Class II objects (see Section 4). The $A_{J}$ values of all these objects besides V892 Tau are below 2.5, and therefore their $n_{5-12}$ and $n_{12-20}$ indices are not fully extinction-free and just approximately indicate their evolutionary state. In addition, 2MASS J04202606+2804089, FN Tau, and UY Aur lie relatively close to the disk region (their $n_{5-12}$ values range from -0.1 to 0.2 ). V892 Tau is a Herbig Ae/Be star with likely 


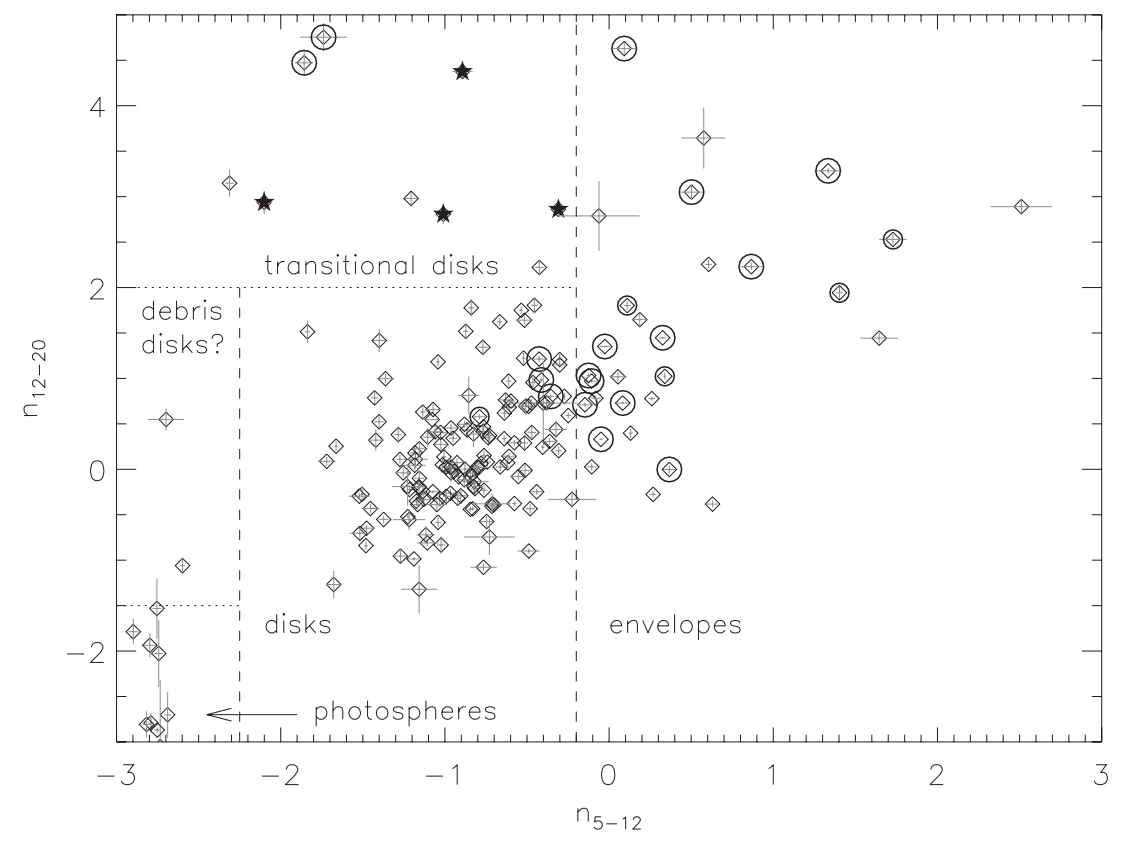

Figure 2. Spectral index between 12 and $20 \mu \mathrm{m}$ vs. the spectral index between 5 and $12 \mu \mathrm{m}$ for all YSOs in Taurus from Furlan et al. (2006, 2008) and this work. Previously known transitional disks, i.e., disks with dust-depleted inner regions (CoKu Tau/4, DM Tau, GM Aur, UX Tau A), are shown with star symbols; protostars surrounded by envelopes and studied in Furlan et al. (2008) are plotted with circled diamonds (the smaller circles denote sources with more tenuous envelopes). Objects in the lower left corner are Class III objects.

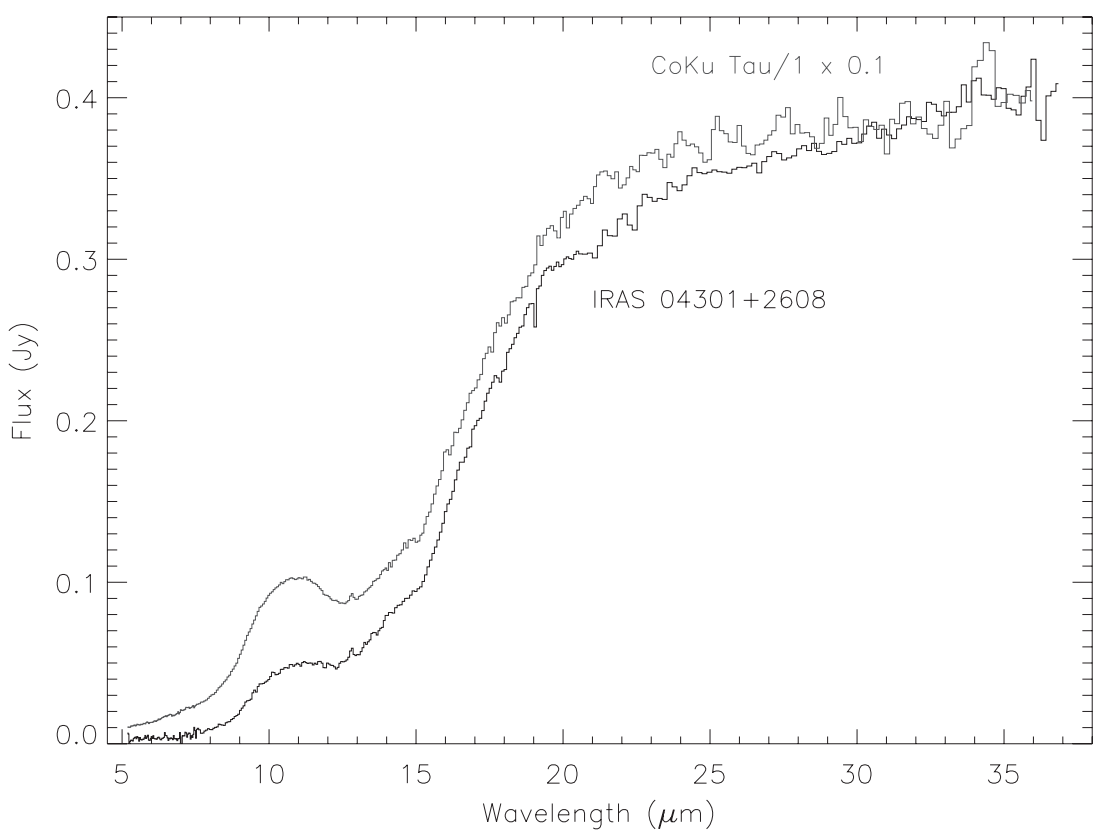

Figure 3. IRS spectra of IRAS 04301+2608 (black) and CoKu Tau/1 (gray).

a different disk structure than that of the lower-mass $\mathrm{T}$ Tauri stars. Haro 6-5B is surrounded by an edge-on disk; its SED is discussed in more detail in Section 4.2.

A few of the Class I objects modeled in Furlan et al. (2008, IRAS 04154+2823, 04365+2535, 04489+3042, and HL Tau), lie in the disk region, but, except for IRAS 04154+2823, their $n_{5-12}$ value is around -0.4 and therefore close to the boundary to the envelope region. The SED of IRAS $04154+2823$ was modeled with a low-density envelope in Furlan et al. (2008), but its extinction-free indices suggest that it is a diskdominated source (see also Section 4.1). This applies also to the other Class I/II objects from Furlan et al. (2008), IRAS $04278+2253$, LkHa 358, and IC 2087 IR. Only one of the
Class I objects added in this work, IRAS 04191+1523, lies in the disk region, but misses the envelope region by a small amount. Once again, this suggests that the "extinction-free" indices are just an approximation for Taurus, which in general suffers from little foreground extinction, but nevertheless they identify the majority of envelope- and disk-dominated objects.

\subsection{Extinction Calculations and Corrections}

Using data from the literature and near-infrared spectra obtained with SpeX (Rayner et al. 2003) at the NASA Infrared Telescope Facility (IRTF), we compiled spectral types and 
Table 4

"Extinction-free" Spectral Indices for all YSOs with IRS Spectra in Taurus

\begin{tabular}{|c|c|c|}
\hline $\begin{array}{l}\text { Name } \\
\text { (1) }\end{array}$ & $\begin{array}{c}n_{5-12} \\
(2)\end{array}$ & $\begin{array}{c}n_{12-20} \\
\text { (3) }\end{array}$ \\
\hline 2MASS J04141188+2811535 & -0.51 & 0.29 \\
\hline 2MASS J04153916+2818586 & -1.43 & 0.79 \\
\hline 2MASS J04155799+2746175 & -1.03 & -0.32 \\
\hline 2MASS J04163911+2858491 & -1.52 & -0.70 \\
\hline 2MASS J04201611+2821325 & -1.02 & -0.83 \\
\hline 2MASS J04202144+2813491 & -0.73 & -0.75 \\
\hline 2MASS J04202606+2804089 & 0.19 & 1.65 \\
\hline 2MASS J04214631+2659296 & -0.82 & -0.14 \\
\hline 2MASS J04230607+2801194 & -1.16 & 0.23 \\
\hline 2MASS J04242090+2630511 & -0.80 & 0.03 \\
\hline 2MASS J04242646+2649503 & -0.88 & -0.12 \\
\hline 2MASS J04263055+2443558 & -1.16 & -1.32 \\
\hline 2MASS J04284263+2714039 & -1.72 & 0.09 \\
\hline 2MASS J04290068+2755033 & -0.93 & 0.07 \\
\hline 2MASS 04293209+2430597 & -0.11 & 0.03 \\
\hline 2MASS J04295950+2433078 & -1.18 & 0.04 \\
\hline 2MASS J04322415+2251083 & -0.75 & -0.57 \\
\hline 2MASS J04330945+2246487 & -1.68 & -1.27 \\
\hline 2MASS J04333905+2227207 & -0.77 & 1.34 \\
\hline 2MASS J04334465+2615005 & -1.07 & -0.25 \\
\hline 2MASS J04335245+2612548 & -0.51 & -0.01 \\
\hline 2MASS J04361030+2159364 & -0.83 & 0.39 \\
\hline 2MASS J04381486+2611399 & -0.52 & 1.22 \\
\hline 2MASS J04390163+2336029 & -1.25 & -0.04 \\
\hline 2MASS J04390396+2544264 & -0.70 & -0.39 \\
\hline 2MASS J04393364+2359212 & -0.79 & 0.02 \\
\hline 2MASS J04400067+2358211 & -1.08 & 0.55 \\
\hline 2MASS J04414489+2301513 & -1.22 & -0.55 \\
\hline 2MASS J04442713+2512164 & -0.31 & 0.20 \\
\hline 2MASS J04554535+3019389 & -1.19 & -0.99 \\
\hline 2MASS J04554801+3028050 & -0.71 & -0.41 \\
\hline AA Tau & -0.92 & -0.08 \\
\hline $\mathrm{AB}$ Aur & -0.87 & 1.52 \\
\hline Anon 1 & -2.79 & -2.79 \\
\hline BP Tau & -0.74 & 0.35 \\
\hline CIDA 1 & -0.36 & 0.31 \\
\hline CIDA 7 & -0.61 & 0.97 \\
\hline CIDA 9 & -0.878 & 0.00 \\
\hline CIDA 11 & -1.11 & -0.81 \\
\hline CIDA 12 & -0.71 & -0.38 \\
\hline CIDA 14 & -1.12 & -0.72 \\
\hline CI Tau & -1.02 & 0.27 \\
\hline CoKu Tau/1 & 1.40 & 1.94 \\
\hline $\mathrm{CoKu} \mathrm{Tau} / 3$ & -1.04 & -0.58 \\
\hline $\mathrm{CoKu} \mathrm{Tau/4}$ & -0.89 & 4.38 \\
\hline CW Tau & -1.16 & -0.20 \\
\hline CX Tau & -0.38 & 0.73 \\
\hline CY Tau & -1.48 & -0.84 \\
\hline CZ Tau & 0.63 & -0.38 \\
\hline DD Tau & -0.76 & -0.23 \\
\hline DE Tau & -0.96 & 0.46 \\
\hline DF Tau & -1.48 & -0.65 \\
\hline DG Tau & -0.25 & 0.59 \\
\hline DG Tau B & 0.37 & -0.00 \\
\hline DH Tau & -1.84 & 1.51 \\
\hline DK Tau & -1.11 & 0.35 \\
\hline DL Tau & -0.85 & -0.44 \\
\hline DM Tau & -0.31 & 2.86 \\
\hline DN Tau & -0.66 & 0.03 \\
\hline DO Tau & -0.64 & 0.34 \\
\hline DP Tau & -0.49 & 0.69 \\
\hline DQ Tau & -0.55 & -0.08 \\
\hline DR Tau & -0.94 & -0.02 \\
\hline DS Tau & -1.13 & -0.33 \\
\hline FF Tau & -2.69 & -2.70 \\
\hline
\end{tabular}

Table 4

(Continued)

\begin{tabular}{|c|c|c|}
\hline $\begin{array}{l}\text { Name } \\
\text { (1) }\end{array}$ & $\begin{array}{c}n_{5-12} \\
(2)\end{array}$ & $\begin{array}{c}n_{12-20} \\
\text { (3) }\end{array}$ \\
\hline FM Tau & -0.61 & 0.68 \\
\hline FN Tau & 0.13 & 0.40 \\
\hline FO Tau & -0.73 & 0.37 \\
\hline FP Tau & -0.95 & 0.34 \\
\hline FQ Tau & -1.16 & -0.10 \\
\hline FR Tau & -0.49 & -0.90 \\
\hline FS Tau & -0.64 & 0.62 \\
\hline FT Tau & -0.74 & 0.08 \\
\hline FV Tau & -0.83 & -0.04 \\
\hline FX Tau & -1.13 & 0.63 \\
\hline FY Tau & -1.50 & -0.28 \\
\hline FZ Tau & -1.17 & -0.39 \\
\hline GG Tau & -0.87 & 0.43 \\
\hline GH Tau & -1.28 & 0.38 \\
\hline GI Tau & -0.62 & 0.07 \\
\hline [GKH94] 41 & -0.52 & 1.64 \\
\hline GK Tau & -0.63 & 0.76 \\
\hline GM Aur & -1.01 & 2.81 \\
\hline GM Tau & -1.16 & -0.19 \\
\hline GN Tau & -0.96 & -0.04 \\
\hline GO Tau & -0.88 & 0.50 \\
\hline GV Tau & 0.27 & -0.28 \\
\hline Haro 6-5B & 0.26 & 0.78 \\
\hline Haro 6-13 & -0.67 & 1.62 \\
\hline Haro 6-28 & -0.96 & 0.03 \\
\hline Haro 6-37 & -1.22 & -0.22 \\
\hline HBC 356 & -2.97 & -5.67 \\
\hline HBC 388 & -2.76 & -4.61 \\
\hline HBC 392 & -2.88 & -6.49 \\
\hline HD 283572 & -2.83 & -3.19 \\
\hline HH 30 & -0.40 & 0.73 \\
\hline HK Tau & -0.84 & 1.78 \\
\hline HL Tau & -0.41 & 0.98 \\
\hline HN Tau & -0.77 & 0.42 \\
\hline HO Tau & -1.01 & 0.14 \\
\hline HP Tau & -0.47 & 0.73 \\
\hline HQ Tau & -0.77 & 0.47 \\
\hline IC 2087 IR & -1.23 & -0.52 \\
\hline IRAS 04016+2610 & 0.08 & 0.73 \\
\hline IRAS $04108+2803 \mathrm{~A}$ & -0.27 & 0.80 \\
\hline IRAS $04108+2803 B$ & -0.03 & 1.35 \\
\hline IRAS 04108+2910 & -1.17 & -0.35 \\
\hline IRAS 04111+2800G & 0.58 & 3.64 \\
\hline IRAS 04125+2902 & -2.31 & 3.15 \\
\hline IRAS 04154+2823 & -0.79 & 0.58 \\
\hline IRAS $04158+2805$ & -0.15 & 0.71 \\
\hline IRAS $04166+2706$ & 0.50 & 3.05 \\
\hline IRAS 04169+2702 & 0.33 & 1.45 \\
\hline IRAS $04173+2812$ & -0.61 & 0.14 \\
\hline IRAS $04181+2654 \mathrm{~A}$ & -0.05 & 0.33 \\
\hline IRAS $04181+2654 B$ & -0.54 & 1.75 \\
\hline IRAS 04187+1927 & -0.48 & -0.43 \\
\hline IRAS $04191+1523$ & -0.30 & 1.15 \\
\hline IRAS F04192+2647 & -1.18 & 0.18 \\
\hline IRAS 04196+2638 & -0.76 & 0.15 \\
\hline IRAS 04200+2759 & -0.58 & 0.29 \\
\hline IRAS $04216+2603$ & -0.83 & -0.44 \\
\hline IRAS 04239+2436 & -0.11 & 0.97 \\
\hline IRAS $04248+2612$ & 0.11 & 1.80 \\
\hline IRAS 04260+2642 & -0.46 & 1.80 \\
\hline IRAS $04263+2654$ & -1.03 & 0.41 \\
\hline IRAS 04264+2433 & 1.73 & 2.53 \\
\hline IRAS $04278+2253$ & -0.58 & -0.38 \\
\hline IRAS $04295+2251$ & 0.34 & 1.02 \\
\hline IRAS $04301+2608$ & 2.51 & 2.89 \\
\hline
\end{tabular}


Table 4

(Continued)

\begin{tabular}{|c|c|c|}
\hline $\begin{array}{l}\text { Name } \\
\text { (1) }\end{array}$ & $\begin{array}{c}n_{5-12} \\
(2)\end{array}$ & $\begin{array}{c}n_{12-20} \\
\text { (3) }\end{array}$ \\
\hline IRAS $04302+2247$ & -1.86 & 4.47 \\
\hline IRAS $04303+2240$ & -0.82 & -0.21 \\
\hline IRAS $04325+2402$ & 0.09 & 4.63 \\
\hline IRAS $04361+2547$ & 1.33 & 3.28 \\
\hline IRAS $04365+2535$ & -0.43 & 1.21 \\
\hline IRAS $04368+2557$ & -1.74 & 4.75 \\
\hline IRAS $04370+2559$ & -0.47 & 0.41 \\
\hline IRAS $04381+2540$ & -0.13 & 1.03 \\
\hline IRAS $04385+2550$ & 0.05 & 1.02 \\
\hline IRAS $04489+3042$ & -0.35 & 0.80 \\
\hline IP Tau & -1.07 & 0.66 \\
\hline IQ Tau & -1.37 & -0.55 \\
\hline IS Tau & -0.90 & -0.29 \\
\hline IT Tau & -1.45 & -0.43 \\
\hline ITG1 & -0.86 & 0.81 \\
\hline ITG 15 & -1.06 & 0.41 \\
\hline ITG 33A & -0.32 & 0.44 \\
\hline ITG 34 & -0.82 & -0.19 \\
\hline ITG 40 & -1.36 & 1.00 \\
\hline IW Tau & -2.81 & -3.67 \\
\hline JH 112 A & -0.51 & 0.69 \\
\hline JH223 & -1.52 & -0.30 \\
\hline KPNO 3 & -0.23 & -0.33 \\
\hline KPNO 7 & -1.23 & -0.19 \\
\hline KPNO 10 & -0.44 & -0.25 \\
\hline KPNO 13 & -1.05 & -0.39 \\
\hline L1551 IRS 5 & 0.87 & 2.23 \\
\hline $\mathrm{LkCa} 1$ & -2.75 & -1.53 \\
\hline $\mathrm{LkCa} 3$ & -2.82 & -2.81 \\
\hline $\mathrm{LkCa} 4$ & -2.73 & -3.05 \\
\hline LkCa 5 & -2.82 & -4.96 \\
\hline LkCa 7 & -2.90 & -1.79 \\
\hline LkCa 15 & -1.40 & 1.42 \\
\hline $\mathrm{LkCa} 21$ & -2.79 & -4.18 \\
\hline LkHa 358 & -1.04 & 1.18 \\
\hline LR 1 & -1.40 & 0.52 \\
\hline MHO 3 & 0.61 & 2.26 \\
\hline MHO 5 & -0.76 & -1.08 \\
\hline MHO 6 & -1.27 & -0.95 \\
\hline RW Aur & -0.78 & 0.06 \\
\hline RX J0432.8+1735 & -2.70 & 0.55 \\
\hline RY Tau & -0.30 & 1.21 \\
\hline SST Tau 041831.2+281617 & -0.06 & 2.79 \\
\hline SU Aur & -0.43 & 2.22 \\
\hline T Tau & -0.60 & 0.751 \\
\hline UX Tau A+C & -2.10 & 2.94 \\
\hline UY Aur & -0.08 & 0.78 \\
\hline UZ Tau A & -1.01 & 0.05 \\
\hline V410 Anon 13 & -0.99 & 0.02 \\
\hline V410 Tau & -2.79 & -3.58 \\
\hline V410 X-ray 1 & -0.84 & -0.08 \\
\hline V410 X-ray 3 & -2.82 & -3.59 \\
\hline V410 X-ray 6 & -1.21 & 2.98 \\
\hline V710 Tau & -0.97 & -0.26 \\
\hline V773 Tau & -0.99 & -0.30 \\
\hline V807 Tau & -1.66 & 0.25 \\
\hline V819 Tau & -2.60 & -1.06 \\
\hline V826 Tau & -2.80 & -1.94 \\
\hline V827 Tau & -2.74 & -2.03 \\
\hline V830 Tau & -2.76 & -7.71 \\
\hline V836 Tau & -1.42 & 0.32 \\
\hline V892 Tau & 1.64 & 1.44 \\
\hline V928 Tau & -2.75 & -2.87 \\
\hline V955 Tau & -0.92 & -0.32 \\
\hline VY Tau & -1.18 & 0.11 \\
\hline
\end{tabular}

Table 4

(Continued)

\begin{tabular}{lcr}
\hline \hline Name & $n_{5-12}$ & $n_{12-20}$ \\
$(1)$ & $(2)$ & $(3)$ \\
\hline XEST 13-010 & -1.27 & 0.11 \\
XZ Tau & -0.40 & 0.24 \\
ZZ Tau & -1.13 & -0.24 \\
ZZ Tau IRS & -0.46 & 0.95 \\
\hline
\end{tabular}

Notes. Column 1 lists the name of the object, Column 2 lists the 5-12 $\mu \mathrm{m}$ spectral index, and Column 3 lists the $12-20 \mu \mathrm{m}$ spectral index.

extinctions for all objects in our sample. For the determination of extinctions, we generally used the Two Micron All Sky Survey (2MASS; Skrutskie et al. 2006) magnitudes and assumed either $J-H$ or $J-K$ photospheric colors (from Luhman et al. 2010). For objects in which the absence of strong excess emission at long wavelengths indicated that the excess at $H$ should be negligible, which particularly applies to late spectral types, we used $J-H$ colors. If the extinction derived from $J-H$ was larger than the extinction from $H-K$ (which would result in a dereddened flux below photospheric levels at $K$ ), we adopted the extinction from $J-K$. If an excess at $H$ was likely based on the colors at longer wavelengths, we either used the slope of the SpeX spectrum at $1 \mu \mathrm{m}$, where available, or assumed intrinsic $J-H$ and $H-K$ colors typical for mid-K to early-M CTTS (the "CTTS locus"; Meyer et al. 1997). The latter method typically yielded extinctions that were within $\pm 0.5 A_{V}$ of the extinctions derived from optical spectra. In the few cases where the differences were larger, we adopted the extinctions derived from the optical spectrum.

For the edge-on disks in our sample (see Section 4.2), no extinctions were estimated, since their near-infrared emission is dominated by scattered light. For the two objects without a known spectral type, IRAS 04370+2559 and ITG 1, we estimated extinctions using SpeX data and $J H K$ colors, respectively. For ITG 1, the near-infrared colors derived from SpeX and 2MASS disagree; dereddening the near-infrared colors to the CTTS locus yields $A_{J}=0.1$, while the colors derived from the SpeX spectrum result in $A_{J}=1.4$. Since the IRS spectrum suggests that little extinction is present (no hint of absorption within the $10 \mu \mathrm{m}$ silicate emission feature), we adopted the former value for $A_{J}$, but note a larger uncertainty in the adopted extinction. For IRAS $04370+2559$, we derived an extinction value by using the slope of the SpeX spectrum at $1 \mu \mathrm{m}$ and assuming a spectral type of early $\mathbf{M}$ for the star. The spectral types and extinctions $A_{J}$ for our targets can be found in Table 1 .

Overall, the determination of the extinction has to be considered an estimate, since it depends on the intrinsic colors or spectrum assumed for the star, which in some cases is quite uncertain. In particular, optical to near-infrared spectra of accreting T Tauri stars often exhibit veiling, in which continuum emission from the disk-to-star accretion flow and the inner disk rim decreases the strength of photospheric absorption lines (see Hartigan et al. 1989; Calvet \& Gullbring 1998; Muzerolle et al. 2003), and this makes it more difficult to determine spectral types and thus intrinsic colors. Veiling also implies that a near-infrared excess is present (see also Cieza et al. 2005), and therefore assuming photospheric $J-H$ colors may yield an overestimate of $A_{J}$ by up to a magnitude, with the largest errors for stars with strong excesses (Fischer et al. 2011). However, as mentioned earlier, we only assumed photospheric $J-H$ colors for objects with a likely negligible $H$-band excess; otherwise we adopted 
an intrinsic near-infrared color based on typical CTTS excesses (if no near-infrared spectrum was available). It is the extinctions derived from the latter method that are more uncertain.

As in Furlan et al. (2009b), we used three different extinction curves to deredden our data: a spline fit to the Mathis $R_{V}=$ 5.0 curve (Mathis 1990) for objects with $A_{J}<0.85$, and the two curves from McClure (2009) for objects with $A_{J} \geqslant 0.85$ (one for $0.85 \lesssim A_{J}<2.5$, one for the $A_{J} \geqslant 2.5$ regime). For objects that suffer from little extinction, the precise shape of the extinction curve does not matter, since corrections to their photometry and spectra are expected to be small. However, there are a few objects in Taurus with relatively high extinction, and in those cases the application of an extinction curve derived for the interstellar medium (ISM) like Mathis's law would not appropriately correct the IRS spectrum (see Furlan et al. 2009b for details). The median $A_{J}$ value for our Taurus sample is 0.62 (which corresponds to $A_{V}=2.2$ assuming Mathis's extinction curve with $R_{V}=3.1$, i.e., $A_{V} / A_{J}=3.55$ ).

\section{SPECTRAL ENERGY DISTRIBUTIONS}

By combining the IRS spectra with near-infrared photometry from the 2MASS Point Source Catalog (Skrutskie et al. 2006) and Spitzer IRAC and MIPS photometry from Luhman et al. (2010), we constructed SEDs for our Taurus sample; they can be seen in Figures 4, 5, and 6. The first figure shows the SEDs of all the Class II objects in our sample except for the edgeon disks, which are shown in Figure 5. Figure 6 displays the SEDs of the seven Class $0 /$ I objects presented here. The data in Figure 4 are corrected for extinction; for objects with $A_{J}>2.5$ both the observed and dereddened data are plotted, so the effect of extinction correction can be seen. The data in Figures 5 and 6 were not dereddened, since for both types of objects the extinction is not caused by material along the line of sight in front of the object, but by circumstellar dust.

The SEDs in Figure 4 also contain the photosphere, which we derived from colors of young stars (Luhman et al. 2010) and usually normalized at the $J$-band. We caution that in cases where an excess at $J$ is likely present, the level of the photosphere is overestimated if the photosphere is normalized to the $J$-band flux (see Cieza et al. 2005); for strongly accreting stars, the excess emission from circumstellar material can be comparable to the flux from the stellar photosphere in this wavelength region (Fischer et al. 2011).

We have included a SpeX spectrum in the SED of AB Aur and have normalized the stellar photosphere to the SED at $0.8 \mu \mathrm{m}$, since AB Aur exhibits strong near-infrared excess emission. Similarly, we have normalized the photosphere for V892 Tau to its $V$-band photometry, as done in Furlan et al. (2006).

We note that the data in the SED for any given object are not contemporaneous, but young stars are known to be variable (e.g., Eiroa et al. 2002; Bary et al. 2009; Muzerolle et al. 2009; Espaillat et al. 2011), and thus the SEDs are susceptible to variability of both the continuum and emission features. For example, the SED of CW Tau seems to indicate a large, unrealistic, near-infrared excess, but instead it probably was fainter when the 2MASS observations were taken (in 1997) than at the time the Spitzer data were taken (during the 2005-2009 time frame). Also, the SpeX spectrum has a different slope from the $J H K$ colors and suggests that a small excess in the $J$-band was present when it was taken. Some of the other objects in our sample show similar characteristics, hinting at variability. In fact, these objects with apparently problematic near-infrared excesses could be a promising sample to look for accretion variability.

Two more objects with seemingly peculiar SEDs deserve some remarks. The low-mass star 2MASS J04210795+2702204 appears to have an unrealistically high infrared excess, but since it is strongly accreting $(\mathrm{EW}(\mathrm{H} \alpha)=442 \AA)$ and displays excess emission in all near-infrared bands (Guieu et al. 2006), it is difficult to estimate the photospheric emission. Due to its strong accretion, it might also be highly variable. The SED shape beyond about $3 \mu \mathrm{m}$ is fairly typical of that of a Class II object. 2MASS J04390525+2337450 has a strong mid-infrared excess, with a steep slope in the $15-20 \mu \mathrm{m}$ region; it was classified as a possible Class I source by Luhman et al. (2010) and as a flatspectrum source by Rebull et al. (2010), who also determined a spectral type of K5. The IRS spectrum suggests that it is a Class II object, given that it has no ice absorption features, but likely a $10 \mu \mathrm{m}$ silicate emission feature (the spectrum only starts at $9.9 \mu \mathrm{m})$. Using the slope of the SpeX spectrum around $1 \mu \mathrm{m}$, we derived an extinction $A_{J}$ of 0.34 for this object, which roughly agrees with the value obtained from dereddening to the CTTS locus $\left(A_{J}=0.48\right)$. Since the SpeX spectrum indicates little excess in the $1-1.25 \mu \mathrm{m}$ region, we normalized the photosphere at the $J$-band, even though a very small excess could be present.

In the following subsections, we will discuss some interesting groups of objects with common properties.

\subsection{Highly Extinguished Objects}

A few Class II objects have extinctions that are larger than $A_{J}=2.5$ : they are, in order of increasing extinction, V892 Tau, IRAS 04385+2550, IRAS 04303+2240, Haro 613, IRAS 04278+2253, IC 2087 IR, IRAS 04154+2823, IRAS 04370+2559, V410 X-ray 2, ITG 40, LR 1, and [GKH94] 41. These objects likely lie behind regions of large foreground extinction.

V892 Tau is a Herbig Ae/Be star and thus one of the few stars in Taurus with an early spectral type. It is surrounded by a circumbinary disk with an inner hole $35 \mathrm{AU}$ in diameter, seen at an inclination angle of $\sim 60^{\circ}$ (Monnier et al. 2008). This relatively high inclination to the line of sight might be responsible for the large extinction seen for this object.

As mentioned in Furlan et al. (2006), IRAS $04385+2550$ has been classified as a protostar by some authors (e.g., Young et al. 2003), but its dereddened SED looks like that of a typical T Tauri star with a roughly flat 3-30 $\mu \mathrm{m}$ SED and 10 and $20 \mu \mathrm{m}$ silicate emission features. Also, recent CO maps by Schaefer et al. (2009) revealed an extended, rotating disk, seen at an inclination angle of $\sim 50^{\circ}$, which could explain the larger extinction of this object.

IRAS 04303+2240, Haro 6-13, and IRAS 04278+2253 have high mass accretion rates (White \& Hillenbrand 2004). The latter object was classified as a Class I/II object in Furlan et al. (2008); since it has no silicate absorption feature, a weak $\mathrm{CO}_{2}$ ice absorption feature, and no extended emission indicative of an envelope, it was considered a very evolved protostar. Applying an extinction correction to the IRS spectrum of IRAS $04278+2253$ restores the $10 \mu \mathrm{m}$ silicate emission feature. Its dereddened SED looks more like that of a typical T Tauri star.

IC 2087 IR was also identified as a Class I/II object by Furlan et al. (2008), given that it is surrounded by a reflection nebulosity, is associated with a molecular outflow, and displays several absorption features in its IRS spectrum. However, its decreasing slope over the infrared spectral range suggests that no extended, even if tenuous, envelope is present. Its spectral 


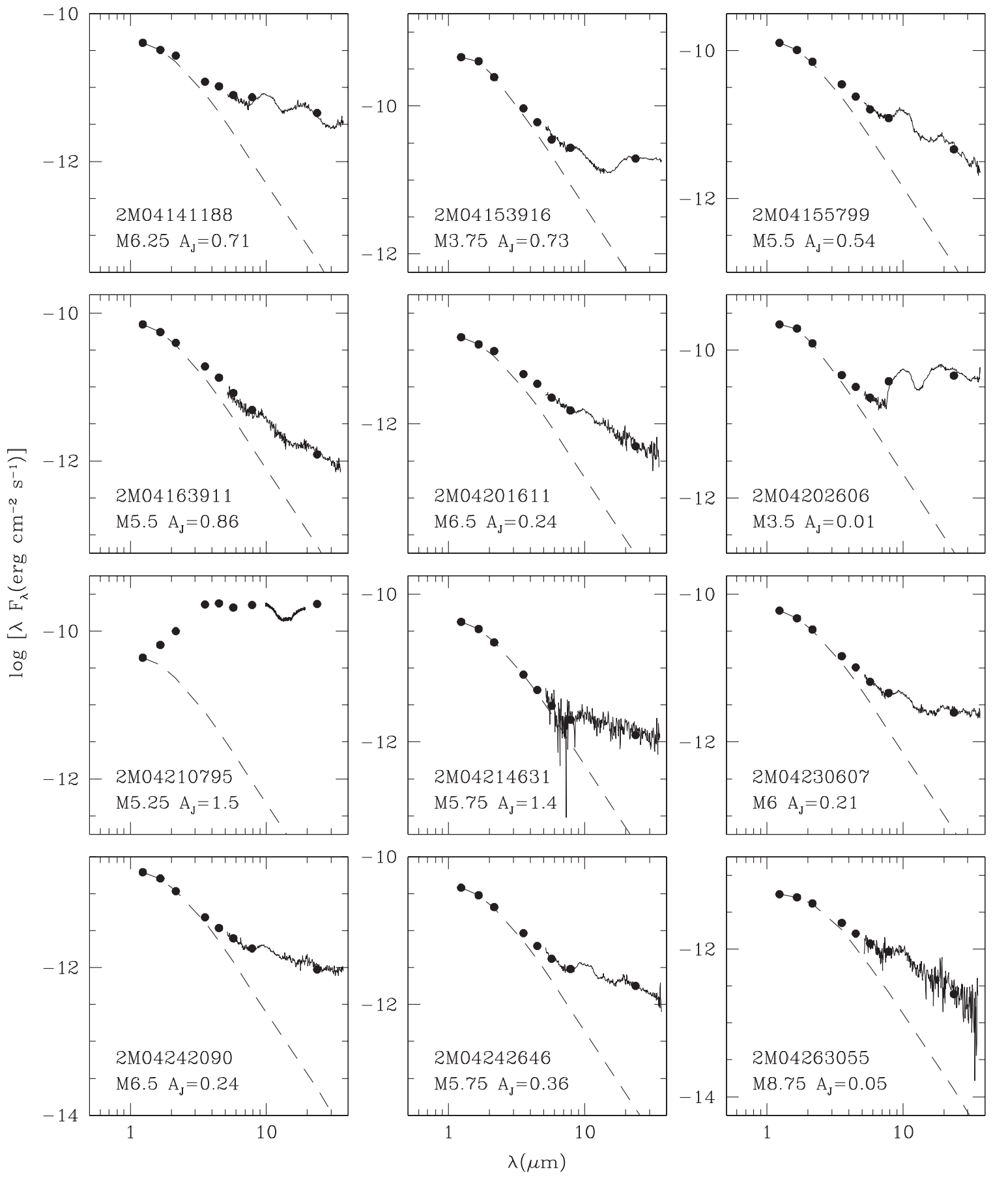

Figure 4. SEDs for the T Tauri stars in Taurus with infrared excesses observed by IRS. The 2MASS, IRAC, and MIPS photometry is shown as filled circles, the IRS spectrum as a solid line, and the photosphere as a dashed line. For objects with $A_{J}>2.5$, we show both the observed and dereddened data (the latter with open circles and dotted lines).

type was inferred to be K by White \& Hillenbrand (2004); due to high optical veiling, it could not be classified more accurately. In the SED plot, we use the colors of a K4 star to estimate the photosphere, following White \& Hillenbrand (2004). We determined the extinction for IC 2087 IR by dereddening the near-infrared colors to the CTTS locus, which yielded $A_{J}=4.1$. This resulted in an SED with a strong near-infrared excess, but some excess already at $J$, as well as scattered light, are possible. Even after the reddening correction, the IRS spectrum of this high-accretion object has a silicate feature that is partly absorbed and a prominent $\mathrm{CO}_{2}$ ice absorption feature. This suggests that some absorbing icy material along the line of sight is probably still present; in fact, Shuping et al. (2001) indicate that the disk around IC 2087 IR is likely seen edge-on, causing the large observed extinction. However, the decreasing mid-infrared SED slope of this object would be very unusual for an edge-on disk (see Section 4.2).

The SED of IRAS $04154+2823$ was identified as that of a flatspectrum source and fitted with a low-density envelope model in Furlan et al. (2008). However, after deriving an extinction $A_{J}=4.2$ from dereddening the $J-H$ color to the CTTS locus and dereddening the photometry and IRS spectrum, the SED is 


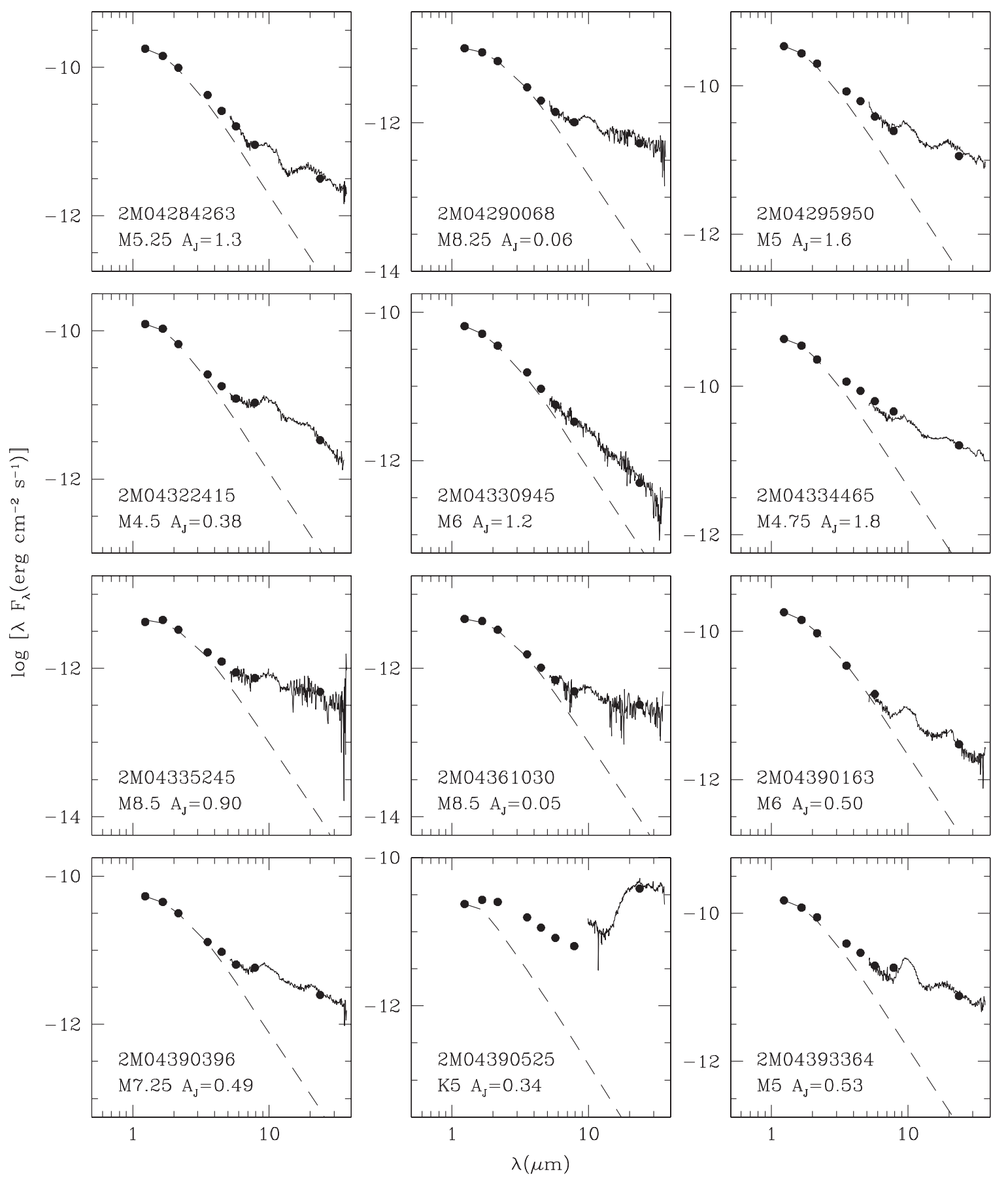

Figure 4. (Continued)

more typical of a T Tauri star, with prominent 10 and $20 \mu \mathrm{m}$ silicate emission features. Only the amount of infrared excess seems too high; in fact, this object has one of the largest $K$-band excesses among the Class II objects in Taurus. Even a larger extinction correction (using $A_{J} \sim 5.7$, derived by assuming photospheric $J-H$ colors) would result in an SED with a large infrared excess. There could be significant contribution of scattered light to the near-infrared emission, implying a higher inclination angle for this object. IRAS $04154+2823$ is also variable; the $J H K$ photometry listed in Kenyon \& Hartmann (1995) is about a magnitude brighter than the 2MASS measurements.
IRAS 04370+2559 also has a mid-infrared excess characteristic of protoplanetary disks, even though its spectral type is not known and therefore the magnitude of the excess can only be crudely estimated. Its extinction was derived from SpeX data; the reddening correction applied to the IRS spectrum results in very pronounced 10 and $20 \mu \mathrm{m}$ silicate emission features and the removal of the weak $\mathrm{CO}_{2}$ ice absorption feature at $15.2 \mu \mathrm{m}$.

V410 X-ray 2, ITG 40, LR 1, and [GKH94] 41 have $A_{J}$ values larger than 6 , which, for an $R_{V}$ value of 3.1 , corresponds to $A_{V} \gtrsim 21$. V410 X-ray 2 has no excess emission out to about $8 \mu \mathrm{m}$, but an excess at longer wavelengths, which is real even when accounting for the background emission that is included 


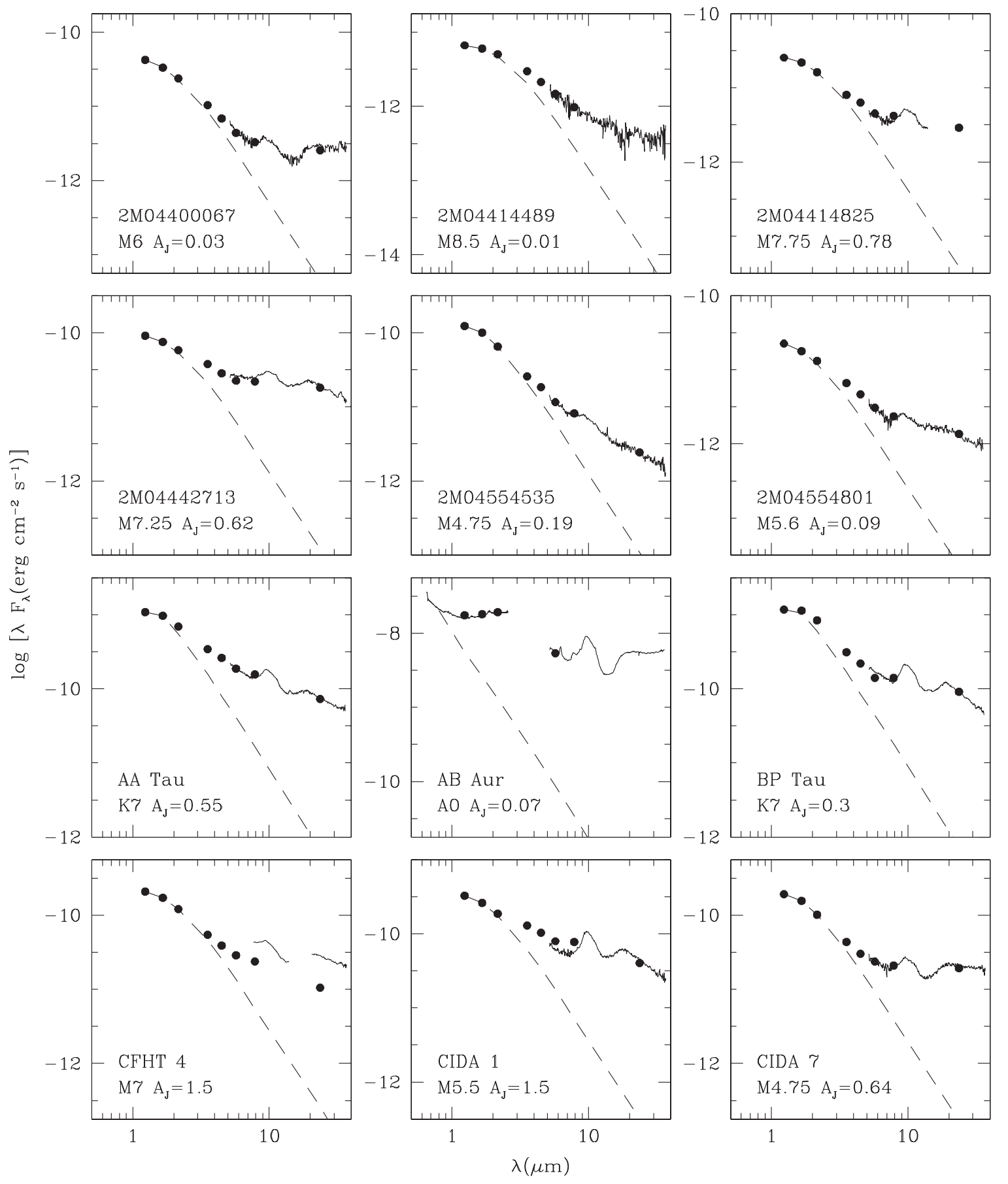

Figure 4. (Continued)

in the IRS flux measurement (see Appendix). In fact, its MIPS $24 \mu \mathrm{m}$ photometry clearly shows an excess at this wavelength (Luhman et al. 2010). The IRS spectrum reveals that it probably has a weak $10 \mu \mathrm{m}$ silicate emission feature. Given the lack of excess at shorter wavelengths and a steep rise of the SED beyond about $15 \mu \mathrm{m}$, this object is reminiscent of a transitional disk. The dereddened SED of ITG 40 shows little excess below about $5 \mu \mathrm{m}$, a small $10 \mu \mathrm{m}$ silicate emission feature, but a very prominent $20 \mu \mathrm{m}$ feature. The SED for LR 1 is similar, but its excess emission is somewhat stronger, and the slope of the SED is flat and not decreasing beyond $20 \mu \mathrm{m}$.

Finally, as already noted by Luhman et al. (2009), [GKH94] 41 is the highest-extinction object among late-type Taurus members (>M6); it is actually the object with the largest extinction in our whole sample of $161 \mathrm{~T}$ Tauri stars. It was discovered in a near-infrared survey by Gomez et al. (1994), where it was suggested to be an embedded protostar due to its very red near-infrared colors and somewhat extended appearance. Without reddening correction, the IRS spectrum of this likely brown dwarf (spectral type of M7.5) displays a $\mathrm{CO}_{2}$ ice absorption feature at $15.2 \mu \mathrm{m}$ and some silicate absorption at $10 \mu \mathrm{m}$. However, the dereddened SED of [GKH94] 41 looks like that of a typical $\mathrm{T}$ Tauri star, with prominent 10 and $20 \mu \mathrm{m}$ silicate emission features and no ice absorption features, indicating that it is surrounded by a disk and not by an envelope. Thus, [GKH94] 41 is a heavily extinguished Class II object. 


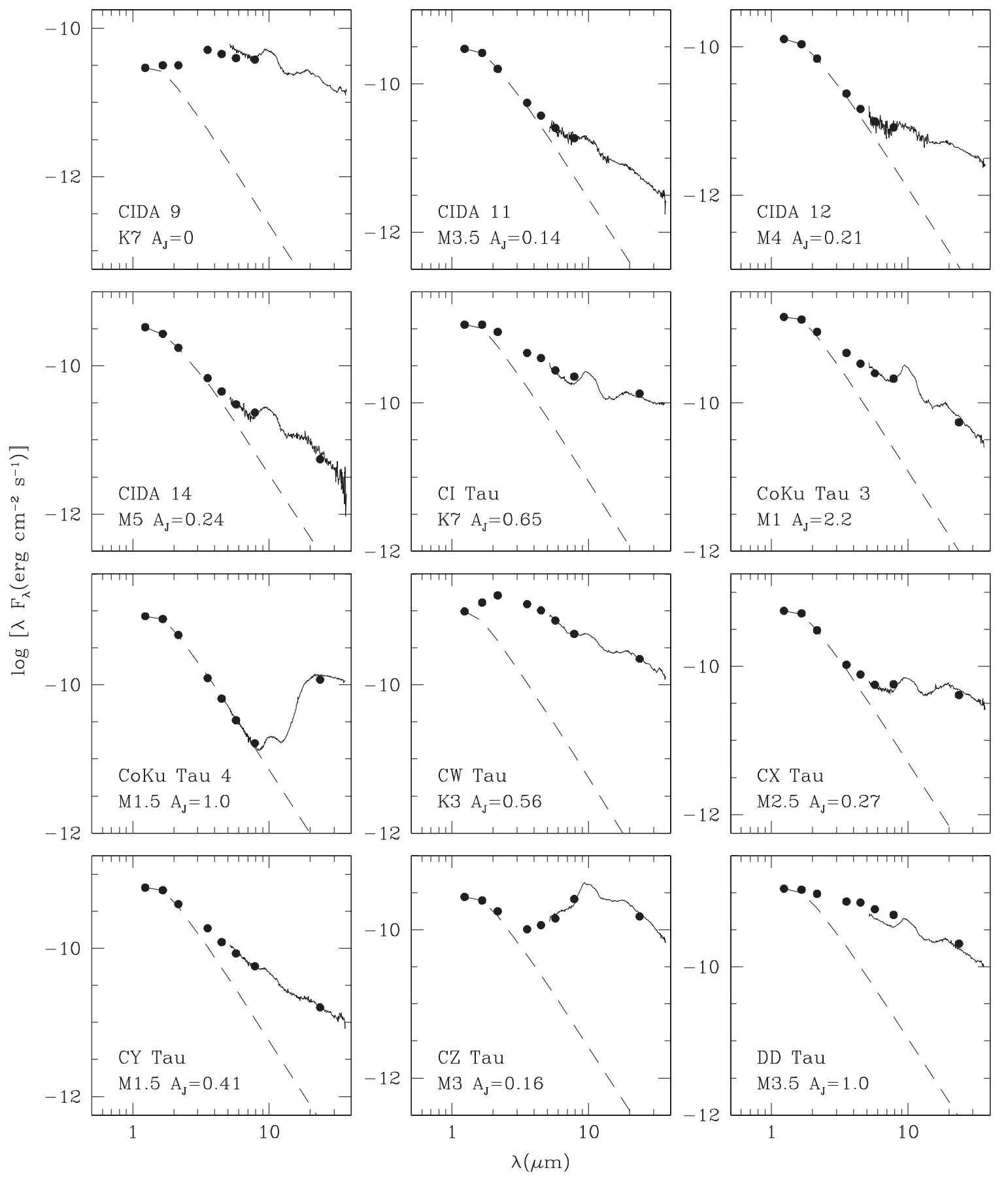

Figure 4. (Continued)

\subsection{Edge-on Disks}

Seven objects in our sample are known or are strongly suspected to have edge-on disks: 2MASS J04202144+2813491 and J04333905+2227207 (Luhman et al. 2009), J04381486+ 2611399 (Luhman et al. 2007), Haro 6-5B (Krist et al. 1998), HH 30 (Burrows et al. 1996), IRAS 04260+2642 (Hartmann et al. 2005), and ZZ Tau IRS (White \& Hillenbrand 2004; Guieu et al. 2006). Their SEDs can be seen in Figure 5. In most cases, high-resolution images reveal a disk observed edge-on (2MASS J04202144+2813491, Haro 6-5B, HH 30, IRAS 04260+2642, and 2MASS J04381486+2611399). 2MASS J04333905+2227207 appears underluminous when plotted on an H-R diagram with other Taurus members, sug- gesting that it is seen in scattered light at near-infrared wavelengths and therefore oriented edge-on (bolometric luminosities are underestimated when calculated from near-infrared fluxes dominated by scattered light). ZZ Tau IRS was suggested to be close to edge-on by White \& Hillenbrand (2004) due to the shape of its emission lines.

Most of the SEDs of edge-on objects share common characteristics: they are dominated by faint scattered light at nearinfrared wavelengths, which is sometimes relatively blue, and thermal emission from the disk in the mid-infrared, which is typically responsible for a rise in the SED beyond about $10 \mu \mathrm{m}$. Since 2MASS J04333905+2227207 displays these properties, its classification as an edge-on disk becomes more certain. In some objects, the silicate emission feature shows some 


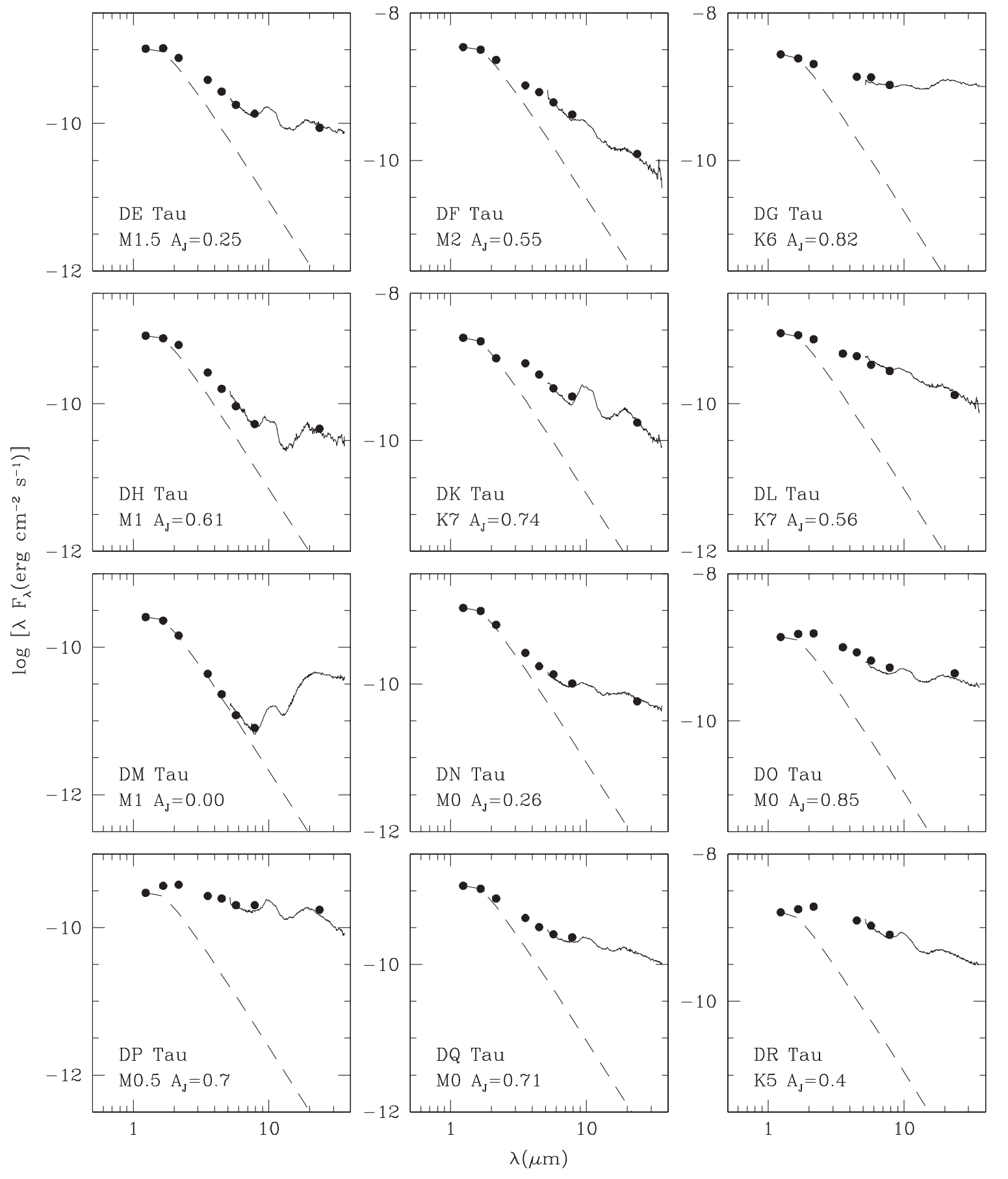

Figure 4. (Continued)

self-absorption due to the cold disk material along the line of sight. The SED of Haro 6-5B stands out, since it looks more like a typical Class I object; the lack of a peak in emission at near-infrared wavelengths could suggest a flared disk seen very close to $90^{\circ}$ inclination.

Another unusual SED among the edge-on disks is that of ZZ Tau IRS, which has silicate emission features and a flat slope over the mid-infrared spectral range, but possibly slight selfabsorption on the short-wavelength side of the $10 \mu \mathrm{m}$ silicate feature. We note that the SED of ZZ Tau IRS in Furlan et al. (2006) was not correct, since it used the 2MASS fluxes of ZZ Tau, which is about an order of magnitude brighter. Using the correct photometry, ZZ Tau IRS has a strong mid-infrared excess. If its SED is dereddened using an $A_{J}$ value of 2.2 (derived by dereddening $J-H$ to the photospheric value), the nearinfrared fluxes seem low compared to the mid-infrared ones, which could be an indication that we are seeing scattered light at near-infrared wavelengths, supporting the idea of an edge-on orientation.

Two more objects in Taurus, CIDA 9 and IRAS 04173+2812, appear underluminous, which could suggest an edge-on orientation, but their mid-infrared SEDs (starting at about $3 \mu \mathrm{m}$ ) look more like those of typical T Tauri stars (see Figure 4).

CIDA 9 is a 2 ". 3 binary, with a primary star that is about a factor of two brighter than the secondary in the $K_{s}$-band (Kraus \& Hillenbrand 2007). Hartmann et al. (2005) noted that the 


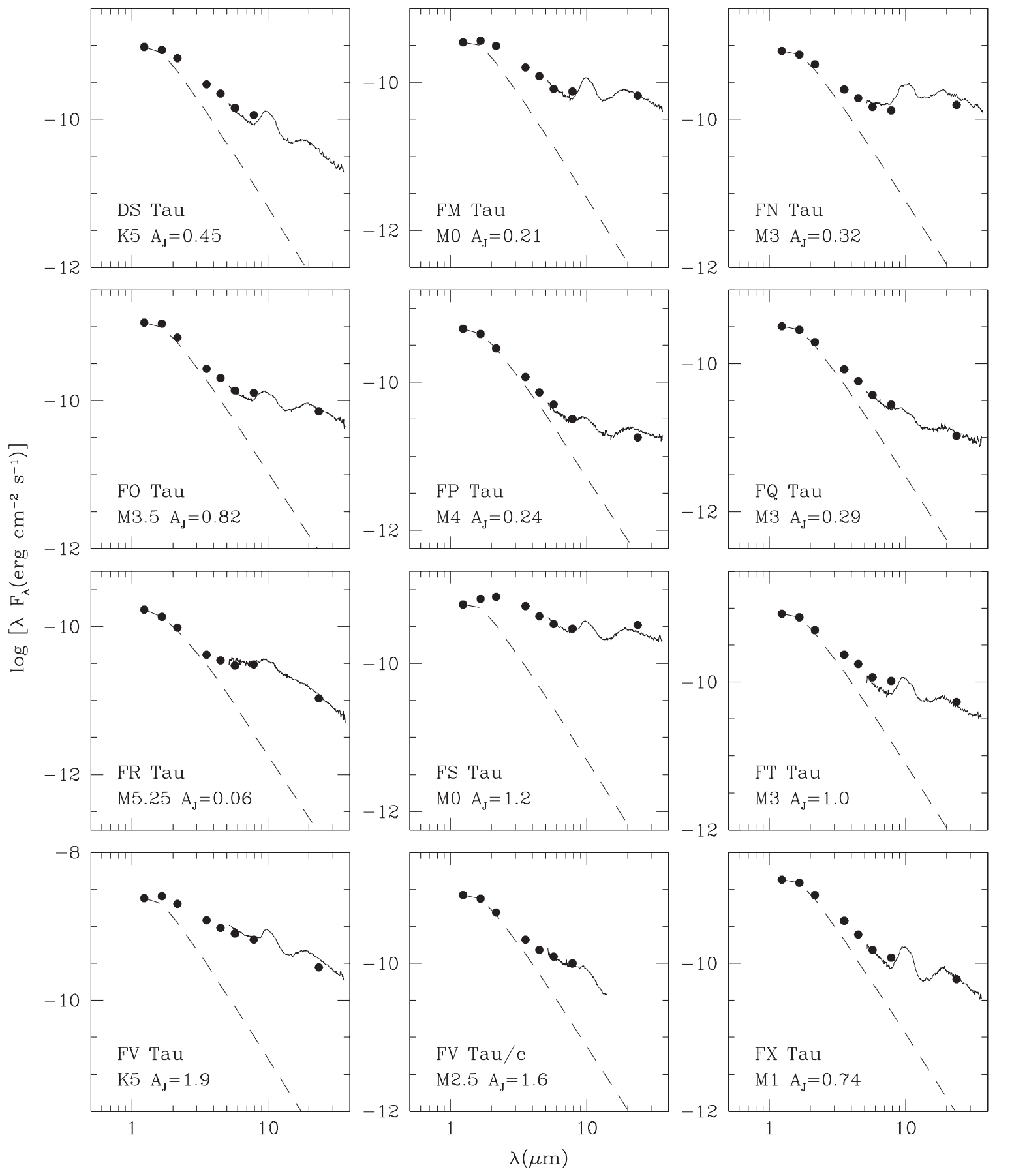

Figure 4. (Continued)

2MASS magnitudes for this object are not consistent with the $J H K$ magnitudes from Kenyon \& Hartmann (1995, the data from 2MASS are 1.3-1.5 mag fainter), but that the latter are more consistent with the IRAC colors. Given that there is also a mismatch between IRAC and IRS data (which were taken in 2004 February and 2009 April, respectively), we conclude that this object must be quite variable and is probably not underluminous.

IRAS $04173+2812$ was identified as a possible edge-on object by Luhman et al. (2009) because it appears underluminous. Rebull et al. (2010) classified it as a flat-spectrum source and derived a spectral type of M4. A larger extinction correction $\left(A_{J} \sim 3.5\right)$ would result in photospheric $J-H$ colors, but still yield a substantial excess at wavelengths longer than $1.65 \mu \mathrm{m}$. In addition, the slope of the SpeX spectrum and the 2MASS $J H K$ colors disagree, with the former being bluer and implying a small extinction of just $A_{J}=1$. Thus, this object is probably highly variable, too, and not oriented close to edge-on.

\subsection{Class I and Class I/II Objects}

The Class I/II objects from Furlan et al. (2008) included in this work are also high-extinction objects (see Section 4.1); LkHa 358 has the lowest extinction among them, $A_{J}=2.4$. Its dereddened SED shows 10 and $20 \mu \mathrm{m}$ silicate emission 


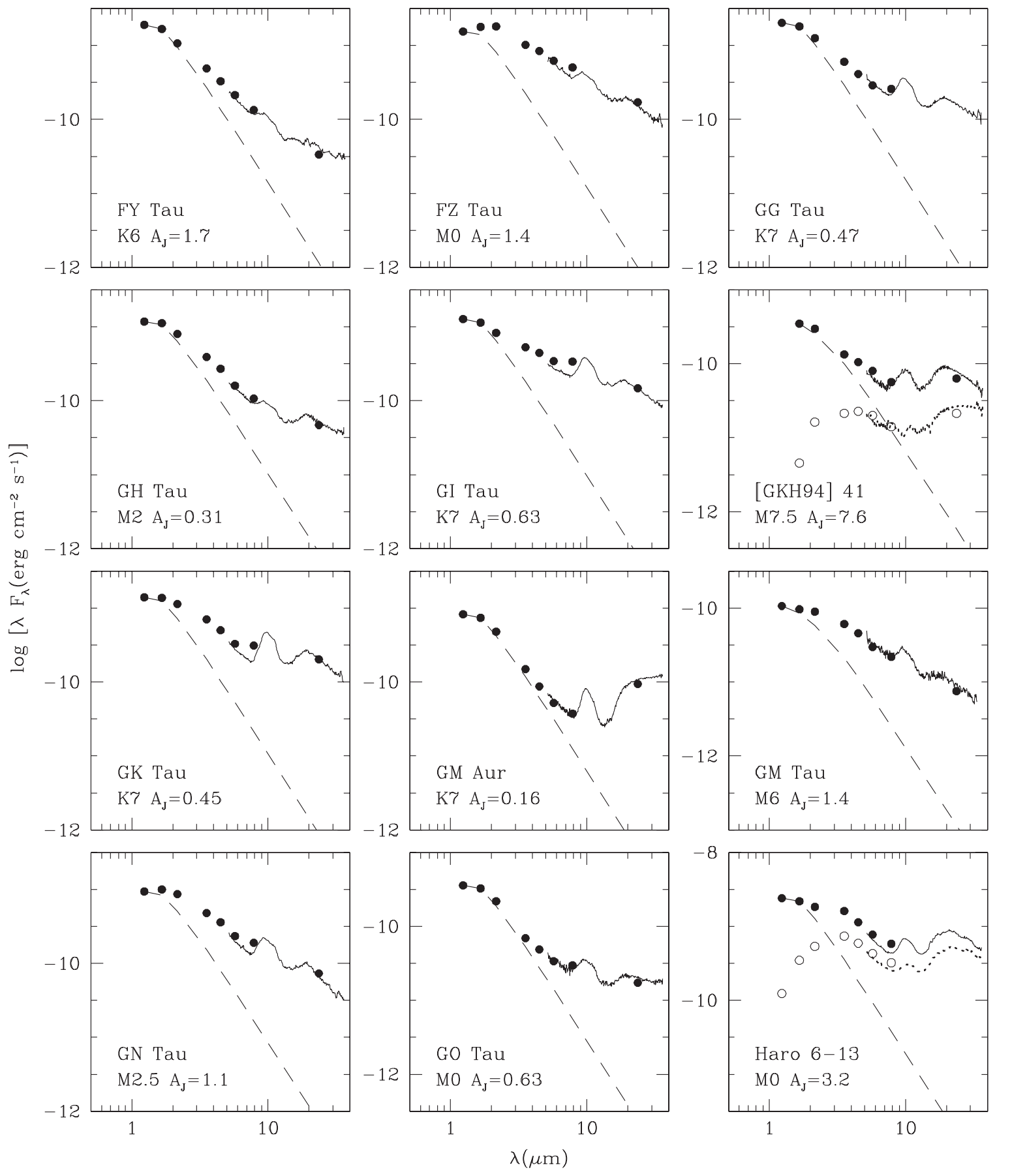

Figure 4. (Continued)

features, and a somewhat steep rise of the IRS spectrum beyond about $12 \mu \mathrm{m}$. As for IRAS 04385+2550, Schaefer et al. (2009) concluded that the $\mathrm{CO}$ emission is generated in a rotating circumstellar disk, seen at an inclination angle of $30^{\circ}$; this disk emission seems to dominate the infrared excess.

Of the seven Class 0/I objects presented here, 2MASS $04293209+2430597$ is the only flat-spectrum source (see Figure 6). Luhman et al. (2009) identified it as a likely protostar based on its very red SpeX spectrum and mid-infrared excess. The other objects have rather steeply rising SEDs over the midinfrared spectral range, which could suggest a more edge-on view for most of them (see Furlan et al. 2008). As mentioned in Section 3.3, IRAS $04301+2608$ is likely surrounded by a tenuous envelope seen at a relatively high inclination angle, like $\mathrm{CoKu}$ $\mathrm{Tau} / 1$. Its $10 \mu \mathrm{m}$ silicate emission feature arises in the inner regions of the low-density envelope. SST Tau 041831.2+281617 is also dominated by an envelope, as derived by its $n_{5-12}$ and $n_{12-20}$ spectral indices and presence of absorption features in the IRS spectrum, and therefore it is confirmed as a new Class I member of Taurus.

Most Class I SEDs show silicate and ice absorption features which are due to the envelopes surrounding them. These features are particularly clear in IRAS $04191+1523$, which is actually a $6^{\prime \prime}$ binary (Duchêne et al. 2004), with the primary brighter than the secondary by more than an order of magnitude (Dunham et al. 2006). 


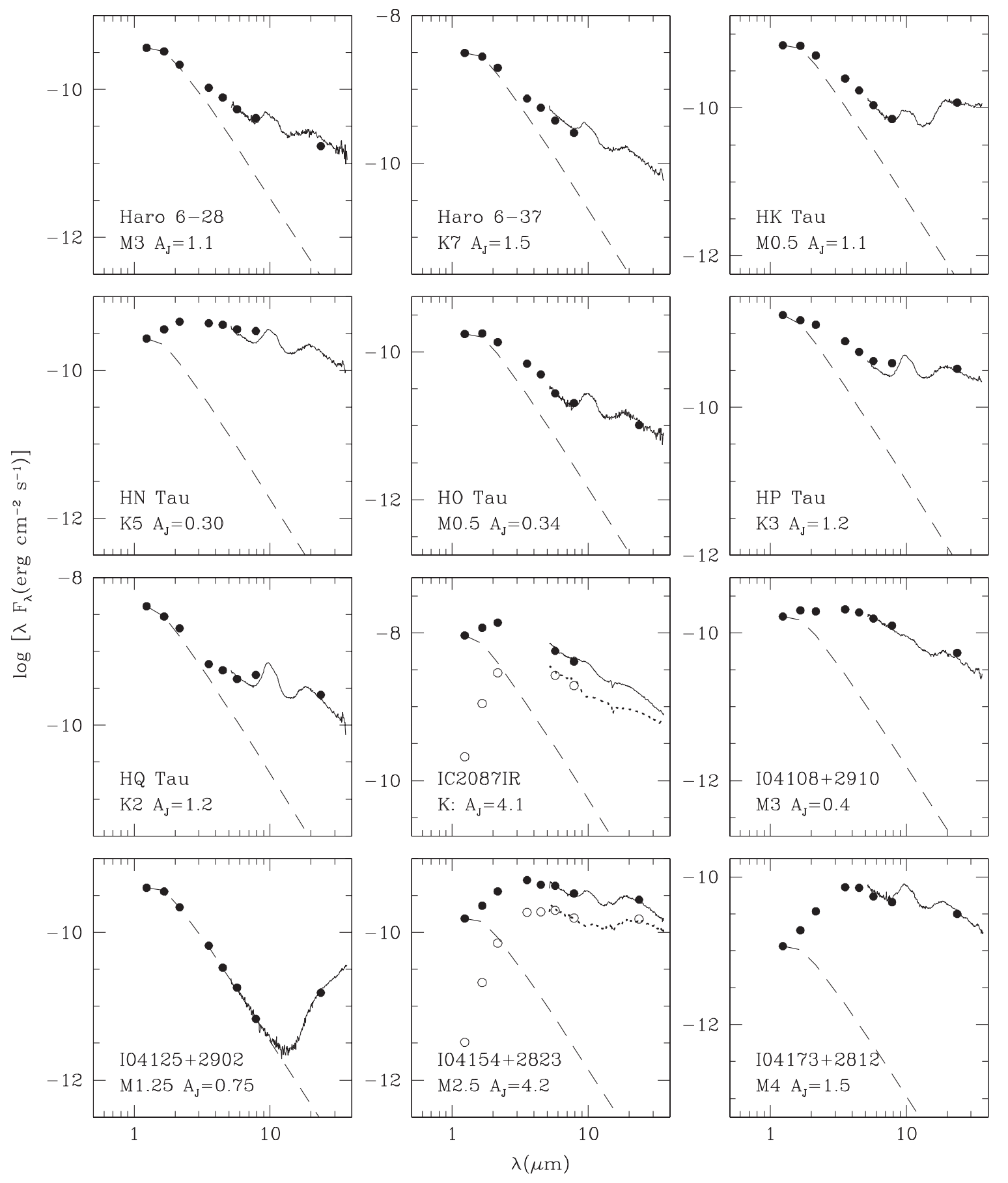

Figure 4. (Continued)

IRAM 04191+1522 and L1521F-IRS are considered very low luminosity objects, since they are embedded in dense cores and have an internal luminosity that is smaller than $0.1 L_{\odot}$ (Dunham et al. 2006; Bourke et al. 2006). They might still accrete substantial amounts of material and become stars, or they could evolve into brown dwarfs. The SEDs of these two objects are similar; both show a steep rise in the infrared, with IRAM 04191+1522 having a steeper slope than L1521F-IRS, and they both have a deep $\mathrm{CO}_{2}$ ice absorption feature at $15.2 \mu \mathrm{m}$.

\subsection{Very Low Mass Stars and Brown Dwarfs}

Of the $161 \mathrm{~T}$ Tauri stars in our sample, 24 have spectral types of M6 or later and therefore are very low mass stars or brown dwarfs. They typically have low excess emission out to about $5 \mu \mathrm{m}$ ( $\lesssim$ a factor of two above the photospheric flux levels), with the notable exception of GM Tau, whose excess seems to start in the $H$-band and whose accretion rate is more typical of higher-mass T Tauri stars (White \& Basri 2003).

2MASS J04381486+2611399 has an unusual SED, with a $10 \mu \mathrm{m}$ silicate feature that is partly self-absorbed. Luhman et al. (2007) determined that it is surrounded by an edge-on disk, making it the only edge-on brown dwarf in our sample. Most of the other brown dwarfs show silicate emission features at 10 and $20 \mu \mathrm{m}$, as are observed in their more massive counterparts, but there are a few objects where these emission features are either very weak or missing (2MASS J04330945+2246487, 2MASS J04335245+2612548, 2MASS J04414489+2301513, KPNO3, 


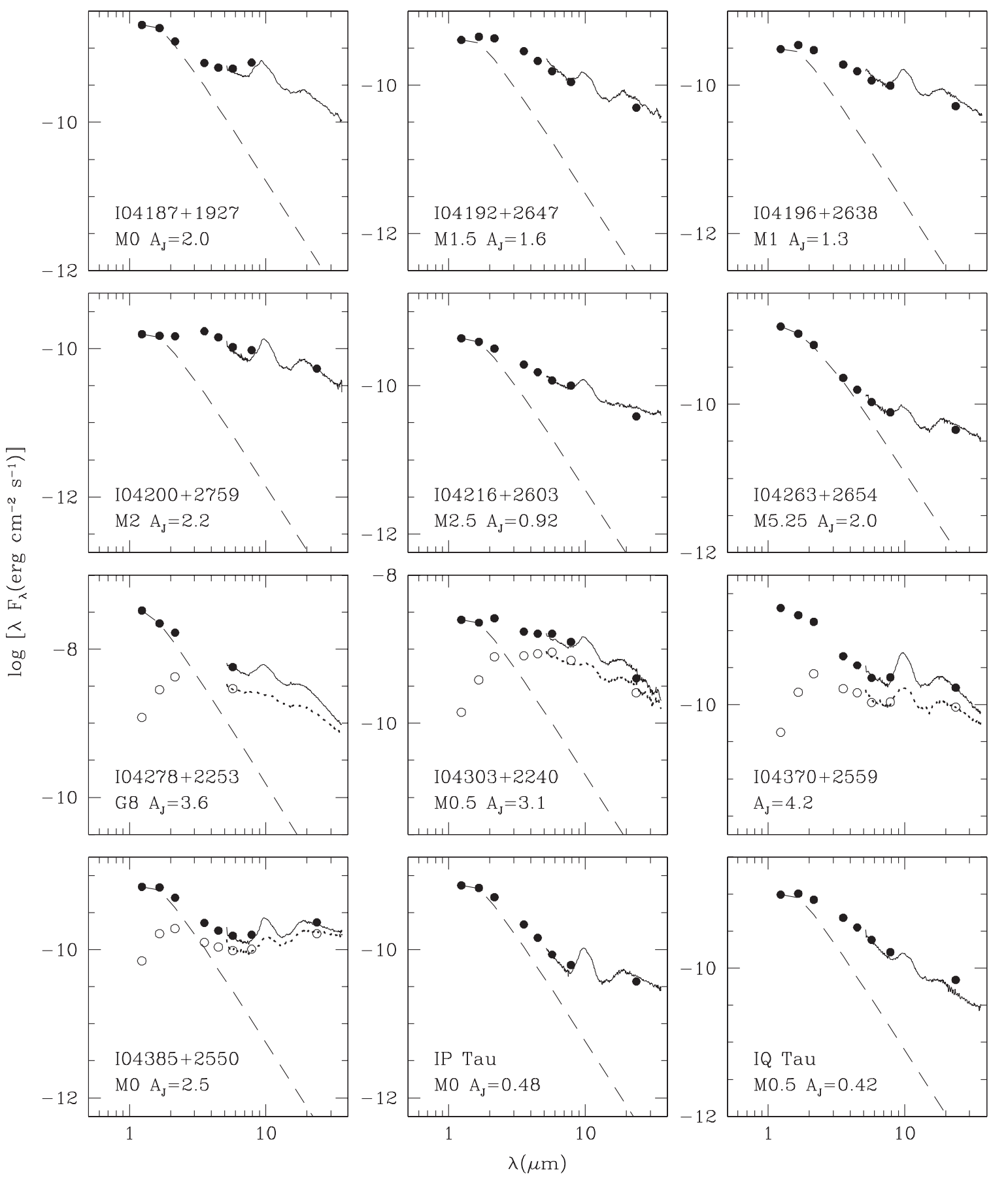

Figure 4. (Continued)

KPNO 6, KPNO 7, and KPNO 12). For these objects, the SEDs typically decrease more steeply over the mid-infrared spectral range as compared to the SEDs of the other brown dwarfs. We will discuss the silicate features in brown dwarf disks in more detail in Section 6.

\subsection{Transitional Disks}

Three transitional disks in Taurus have already been well studied: CoKu Tau/4 (Forrest et al. 2004; D'Alessio et al. 2005; Ireland \& Kraus 2008), DM Tau, and GM Aur (Marsh \& Mahoney 1992; Jensen \& Mathieu 1997; Calvet et al. 2005; Dutrey et al. 2008; Pott et al. 2010). They are characterized by optically thin inner disk regions, suggesting depletion of small dust grains. In addition to the transitional disks, four objects in Taurus have been recognized as pre-transitional disks: LkCa 15, UX Tau A, IP Tau, and RY Tau (Piétu et al. 2006; Espaillat et al. 2007a, 2008, 2010, 2011; Isella et al. 2010; Thalmann et al. 2010). In these objects, a gap separates the optically thick inner disk from the optically thick outer disk.

We note that compared to earlier work, the SED of DM Tau presented here shows a weak excess already at $\sim 5 \mu \mathrm{m}$, while in previous SED versions the excess only started at about $8 \mu \mathrm{m}$ (e.g., Calvet et al. 2005). This is due to our extinction value of $A_{J}=0$ derived from $J-H$ colors (which are photospheric). Adopting a slightly larger extinction value of $A_{J} \sim 0.2$ (Calvet et al. 2005; Espaillat et al. 2011) results in a dereddened $H$-band 


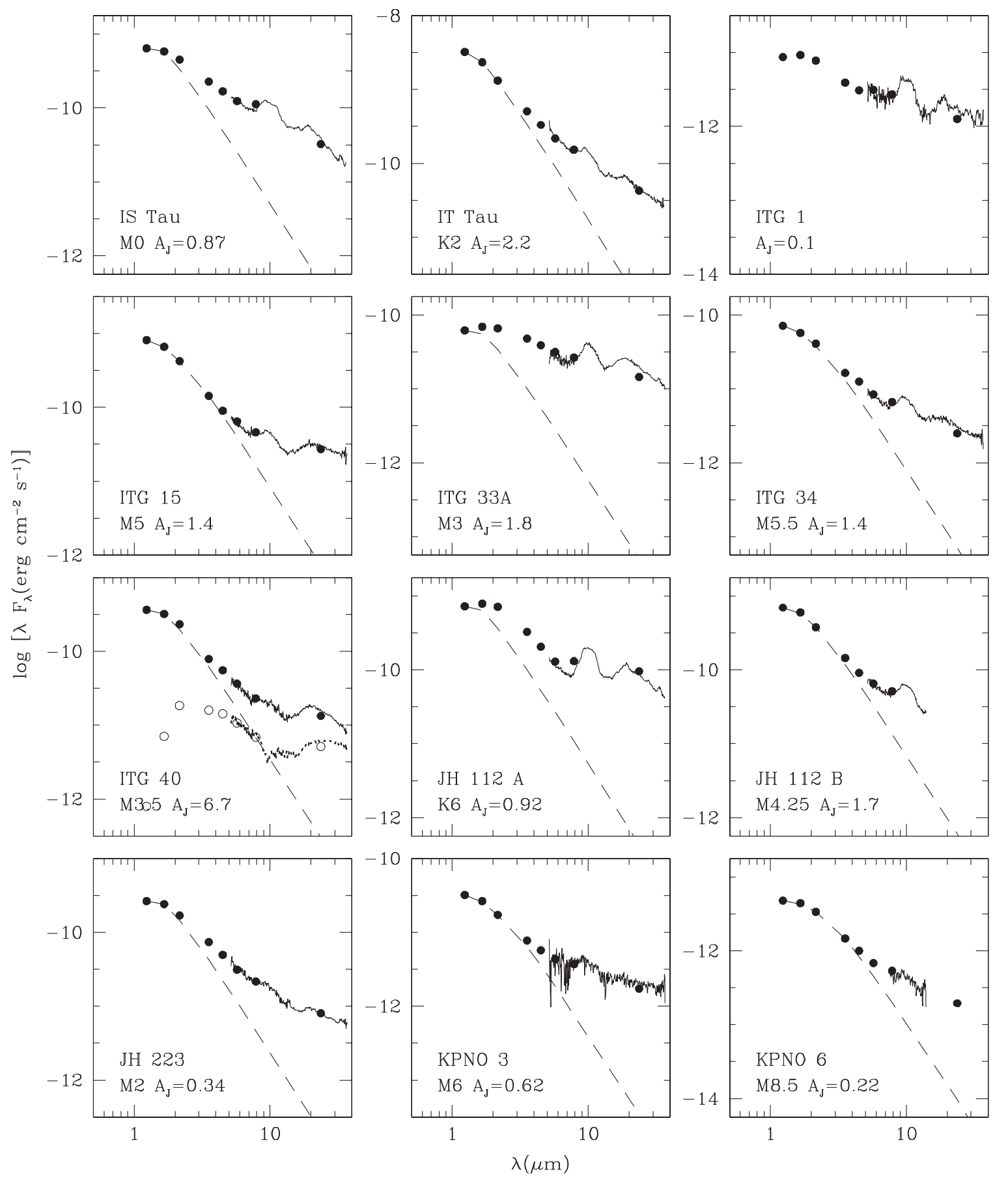

Figure 4. (Continued)

flux somewhat below the photosphere, but no excess emission out to about $8 \mu \mathrm{m}$. Similarly, our newly derived extinction values for LkCa 15 and UX Tau A are smaller than the values adopted in earlier work (e.g., Espaillat et al. 2011), while those for IP Tau and RY Tau are somewhat larger. However, since the extinction values are small, the effect on the SED is relatively minor. The differences in extinction can be understood in terms of the uncertainties involved in deriving them. Since for transitional and pre-transitional disks the excess in the $2-8 \mu \mathrm{m}$ region is typically low due to inner disk clearings, it is particularly important to derive extinction values carefully, since they will have an effect on the amount of excess emission and thus the inferred properties of the inner disk material.
Among the objects whose IRS spectra are presented here for the first time, we point out V410 X-ray 6, whose SED is typical for a transitional disk, with no excess emission out to about $8 \mu \mathrm{m}$, which is indicative of cleared inner disk regions (see also Luhman et al. 2010). With a spectral type of M5.5, it is considered a very low mass star. We note that in the SED plot of V410 X-ray 6 the dereddened fluxes in the 3-8 $\mu \mathrm{m}$ region seem to lie slightly below the photosphere. This could be attributed to variability of the near- or mid-infrared emission, since there is also a small mismatch between the IRAC and IRS data.

Another object that seems to have dust-depleted inner disk regions is IRAS $04125+2902$. Its excess emission starts at about $12 \mu \mathrm{m}$ and rises steeply beyond $15 \mu \mathrm{m}$. Luhman et al. (2009) 


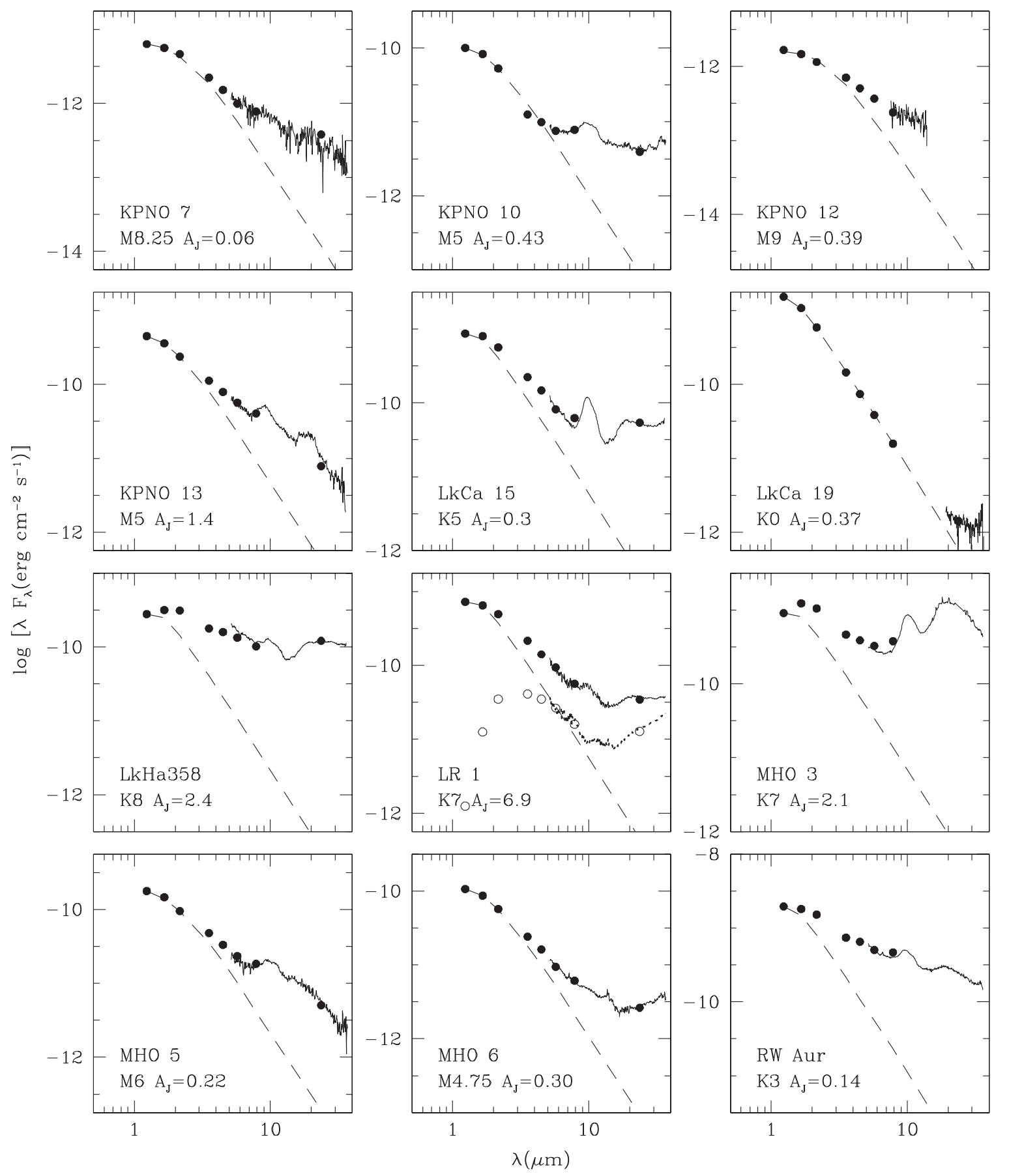

Figure 4. (Continued)

already noted its peculiar SED, with no excess emission in all four IRAC bands, but a clear excess at $24 \mu \mathrm{m}$, and suggested it could be a disk with an inner hole. The lack of a $10 \mu \mathrm{m}$ silicate emission feature sets this object apart from the other transitional disks in Taurus; it is however similar to the transitional disk T25 in Chamaeleon I (Kim et al. 2009; Espaillat et al. 2011), whose SED implies an inner disk hole of $13 \mathrm{AU}$ with only $10^{-13} M_{\odot}$ of small, optically thin dust within $1 \mathrm{AU}$ from the star (Espaillat et al. 2011).

As mentioned earlier (see Section 4.1), V410 X-ray 2 is likely also a transitional disk with an inner hole. 2MASS J04390525+2337450 could be a pre-transitional disk, given that its SED rises steeply beyond $15 \mu \mathrm{m}$, but displays a strong excess already at near-infrared wavelengths. Thus, it could have a gap in its optically thick disk, with the shorter- and longer-wavelength infrared excesses attributable to the disk inside and outside the gap, respectively.

Finally, there are three objects with hardly any excess below $\sim 10 \mu \mathrm{m}$ and only a weak excess at longer wavelengths: LkCa 19, RX J0432.8+1735, and V819 Tau (see also Section 3.3). All three objects are WTTS (White \& Ghez 2001; Wichmann et al. 1996), indicating that their disks are not accreting any more. The infrared excess is weakest for $\mathrm{LkCa}$ 19 and strongest for RX J0432.8+1735, but even in the latter object it likely arises in a remnant, optically thin disk with some inner disk clearing (Furlan et al. 2009a; Padgett et al. 


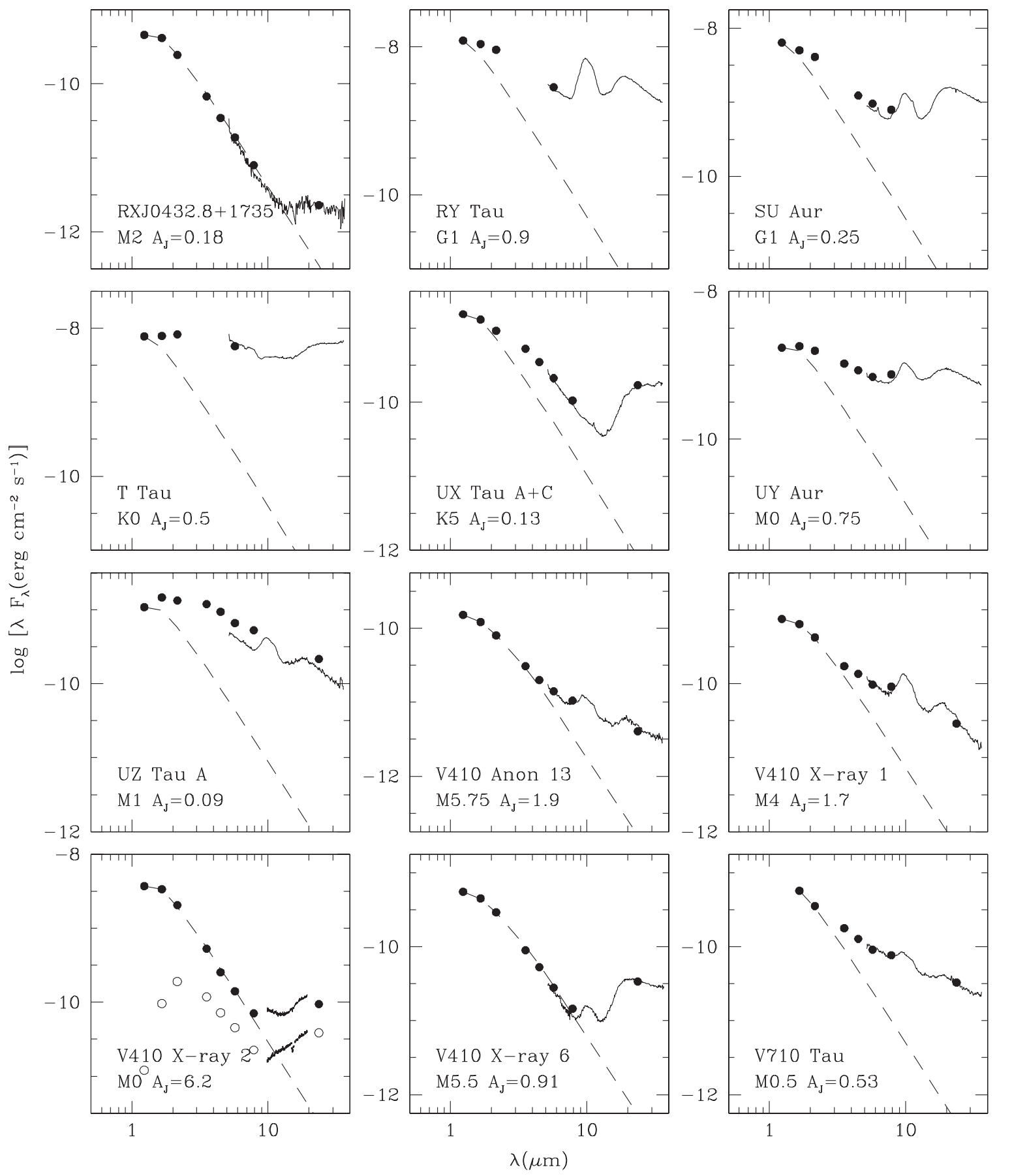

Figure 4. (Continued)

2006). These three objects can be considered transitional disks at an advanced evolutionary stage, since their disks are optically thin with large inner holes, but they could even be young debris disks made of second-generation dust (see Furlan et al. 2009a).

\section{DISK STRUCTURE DERIVED FROM IRS SPECTRA}

As in Furlan et al. (2006), we calculated spectral indices $n\left(\lambda F_{\lambda} \propto \lambda^{n}\right)$ for our sample of Class II objects to measure the slope of the SED between wavelengths that are mostly free of dust or gas emission features and are thus dominated by continuum emission. In this way we get information on disk structure, since the continuum emission depends on the amount of radiation intercepted and reprocessed by the disk, and therefore a more settled disk will have a more negative continuum spectral index than a more flared one (D'Alessio et al. 2006; Furlan et al. 2005, 2006). Here we computed spectral indices between 6 and $13 \mu \mathrm{m}\left(n_{6-13}\right)$ and between 13 and $31 \mu \mathrm{m}$ $\left(n_{13-31}\right)$ in the dereddened spectra; the fluxes at 6,13 , and $31 \mu \mathrm{m}$ were measured by averaging the flux in the 5.4-6.0, 12.8-14.0, and 30.3-32.0 $\mu \mathrm{m}$ regions, respectively.

Figure 7 shows the 6-13 and 13-31 $\mu \mathrm{m}$ spectral indices for our sample of T Tauri stars in Taurus (see also Table 5). Most objects occupy the region where optically thick accretion disks are expected to lie based on theoretical models (delineated by a polygon in the figure). We used models from a grid of accretion disk models (see D'Alessio et al. 1999, 2006; Espaillat 2009) 


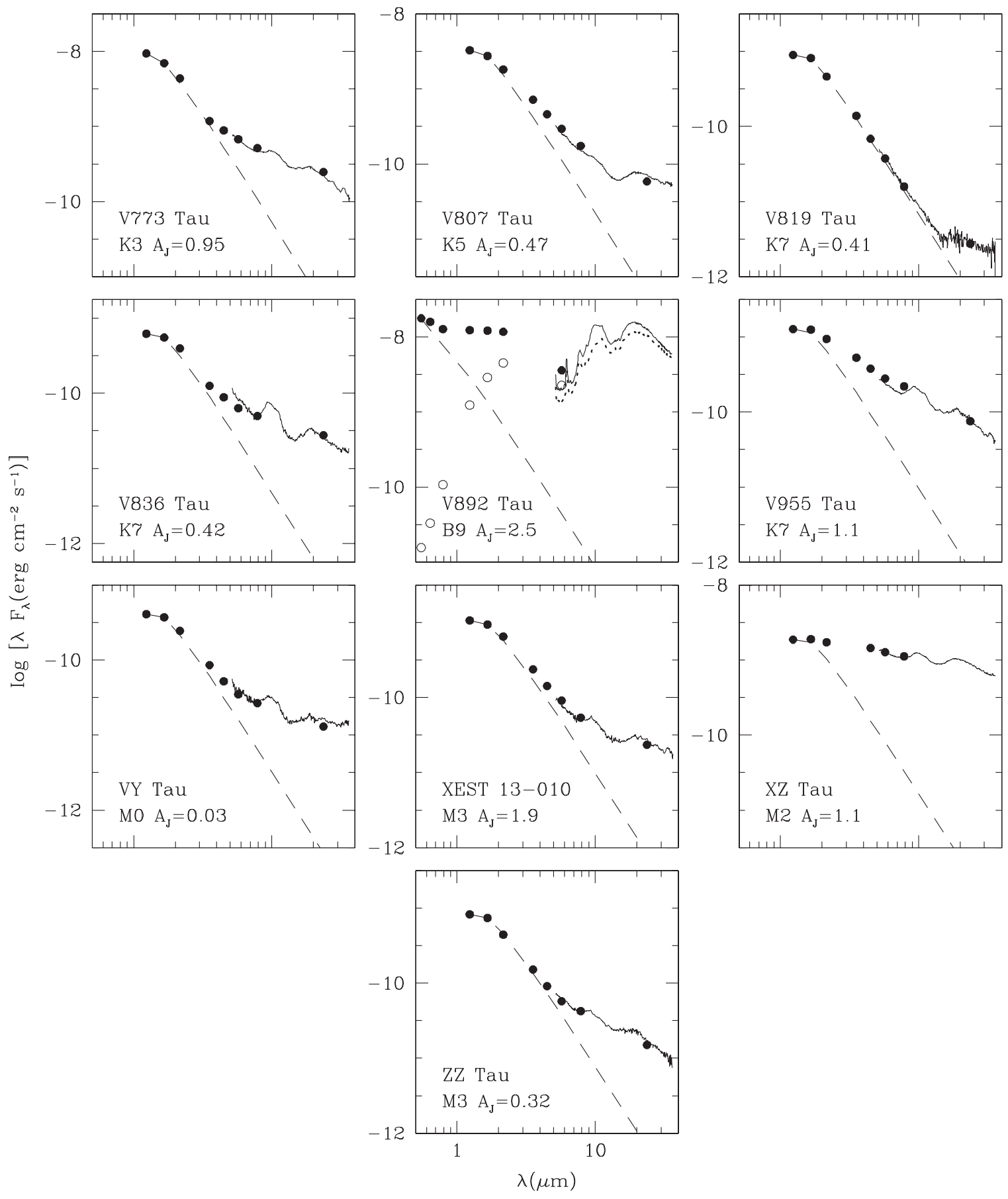

Figure 4. (Continued)

for a $0.5 M_{\odot}$ central star, three different accretion rates $\left(10^{-8}\right.$, $10^{-9}$, and $\left.10^{-10} M_{\odot} \mathrm{yr}^{-1}\right)$, three inclination angles $\left(20^{\circ}, 40^{\circ}\right.$, $\left.60^{\circ}\right)$, and four different settling parameters $\epsilon(0.001,0.01,0.1$, and 1.0), which parameterize the depletion of small dust grains in the upper disk layers relative to the "standard" dust-to-gas mass ratio of $1 / 100$ (i.e., $\epsilon=0.1$ means that the dust in the upper layers is depleted by a factor of 10). These are physical models using input parameters that are physically motivated, mostly derived from observations; the model parameters were chosen to yield models that are representative for the disks in our sample and thus result in plausible estimates of their disk properties. The locations of the objects in Figure 7 imply that most objects have experienced dust settling, since their $n_{13-31}$ values are negative (see D'Alessio et al. 2006; Watson et al. 2009; Espaillat 2009; Furlan et al. 2009b).

There are several "outliers," defined as objects whose spectral indices cannot be described by full accretion disks. Disks with large $n_{13-31}$ spectral indices $(\gtrsim 1.2)$ are usually characterized by inner disk holes; the inner disk rim, exposed directly to the stellar radiation, causes a steep rise of the SED beyond $13 \mu \mathrm{m}$ (Forrest et al. 2004; D'Alessio et al. 2005; Calvet et al. 2005; Espaillat et al. 2007b; Brown et al. 2007; Muzerolle et al. 2010). These disks are usually interpreted to be in a transitional stage of evolution, when the inner disk has already dispersed significantly, but the outer disk remains. However, in a few cases a close binary could be responsible for the inner disk hole 


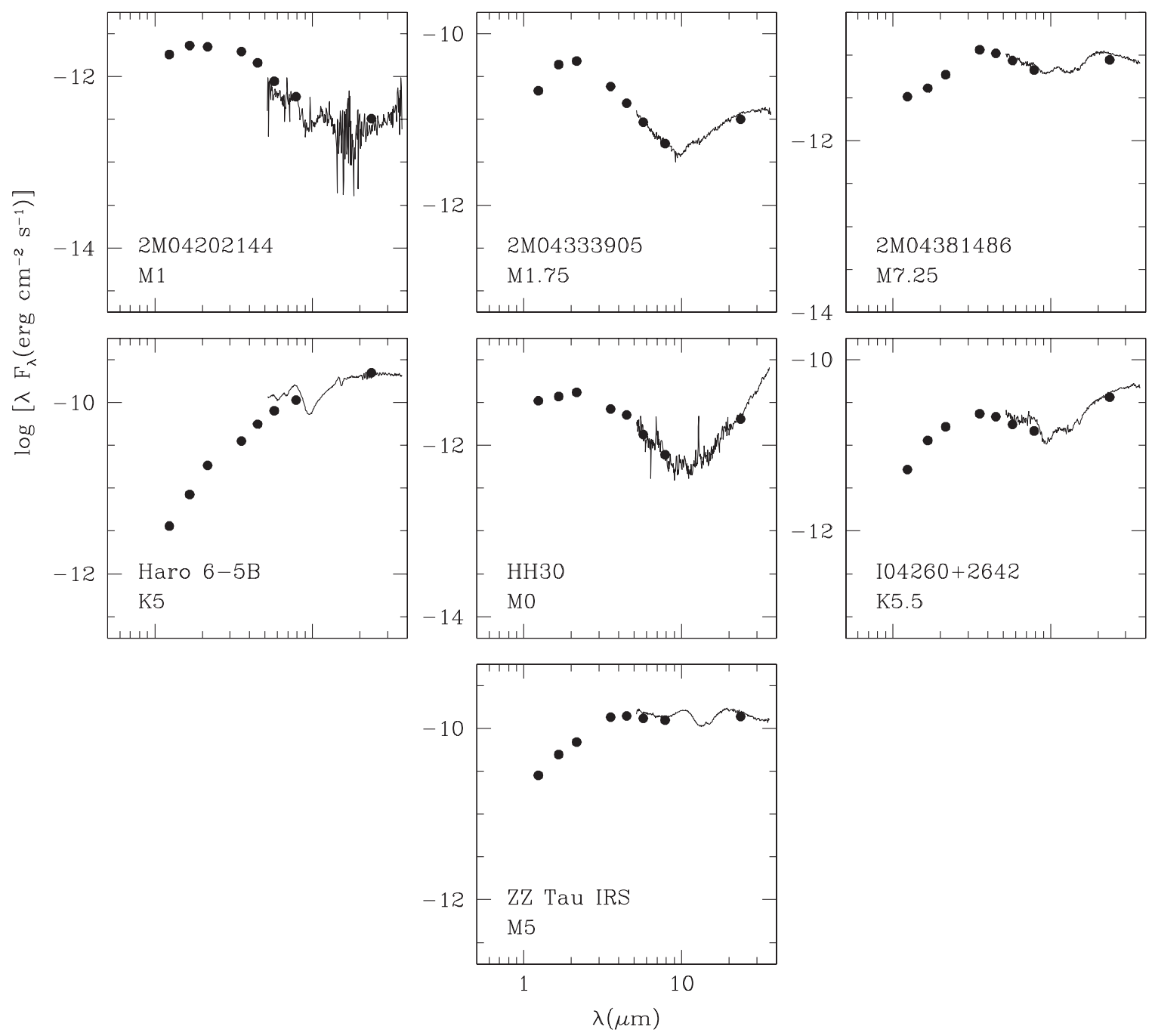

Figure 5. SEDs for the egde-on T Tauri stars in Taurus. The observed 2MASS, IRAC, and MIPS photometry is shown as black, filled circles, and the observed IRS spectrum as a solid line.

(see Ireland \& Kraus 2008), which might then be a longer-lived structure.

In Figure 7, we labeled the transitional disks CoKu Tau/4, DM Tau, and GM Aur, and the pre-transitional disk UX Tau A; they have been identified and studied before through detailed observations and modeling (D'Alessio et al. 2005; Calvet et al. 2005; Espaillat et al. 2007a, 2010, 2011; Ireland \& Kraus 2008; Hughes et al. 2009; Salyk et al. 2009; Pott et al. 2010; Andrews et al. 2011). As already mentioned in Section 4.5, in this work we present the IRS spectra of three additional transitional disks, V410 X-ray 6, IRAS 04125+2902, and V410 X-ray 2. The $n_{13-31}$ value of V410 X-ray 6 is similar to that of DM Tau and thus lies just somewhat above the 13-31 $\mu \mathrm{m}$ slope expected for typical accretion disks without inner disk holes. On the other hand, the $n_{13-31}$ value of IRAS $04125+2902$ is the largest among our sample of Class II objects; the lack of silicate and excess emission out to about $12 \mu \mathrm{m}$ suggests the presence of a large disk hole. The 6-13 and 13-31 $\mu \mathrm{m}$ spectral indices of V410 $\mathrm{X}$-ray 2 could not be calculated (and thus they are not included in Figure 7), since only an $\mathrm{SH}$ spectrum is available for this object.

Also some edge-on disks, namely $\mathrm{HH} 30$ and IRAS $04260+2642$, occupy the region of large $n_{13-31}$ values (see
Figure 7); in these cases, the steep rise of the SED is caused by the emission from the outer disk that is not obscured any more at longer wavelengths. Other properties of the objects, like the shape of the SED at shorter wavelengths, the presence of absorption features, or extinction measurements, are needed to distinguish edge-on from transitional disks.

Only one object has an anomalously large $n_{6-13}$ value: V892 Tau. It is an early-type star whose continuum emission rises steeply from 5 to $20 \mu \mathrm{m}$, then decreases (see also Furlan et al. 2006). As mentioned in Section 4.1, its disk has a relatively large inclination to the line of sight (Monnier et al. 2008). MHO 3 also has a somewhat large 6-13 spectral index; its SED does resemble that of V892 Tau, so its disk might be seen at a relatively large inclination angle, too.

A few objects have $n_{6-13}$ or $n_{13-31}$ values below those inferred from our grid of accretion disk models. Since a spectral index of $-4 / 3$ is indicative of a flat, optically thick disk (e.g., Kenyon \& Hartmann 1987), a steeper slope could reveal optically thin emission, especially if the amount of excess emission with respect to the photosphere is low, or even a truncated disk. 2MASS J04330945+2246487 has a very steep SED dominated by continuum emission; the slope over the entire IRS range is about -1.7 (see Figure 4). This very low mass star or brown 


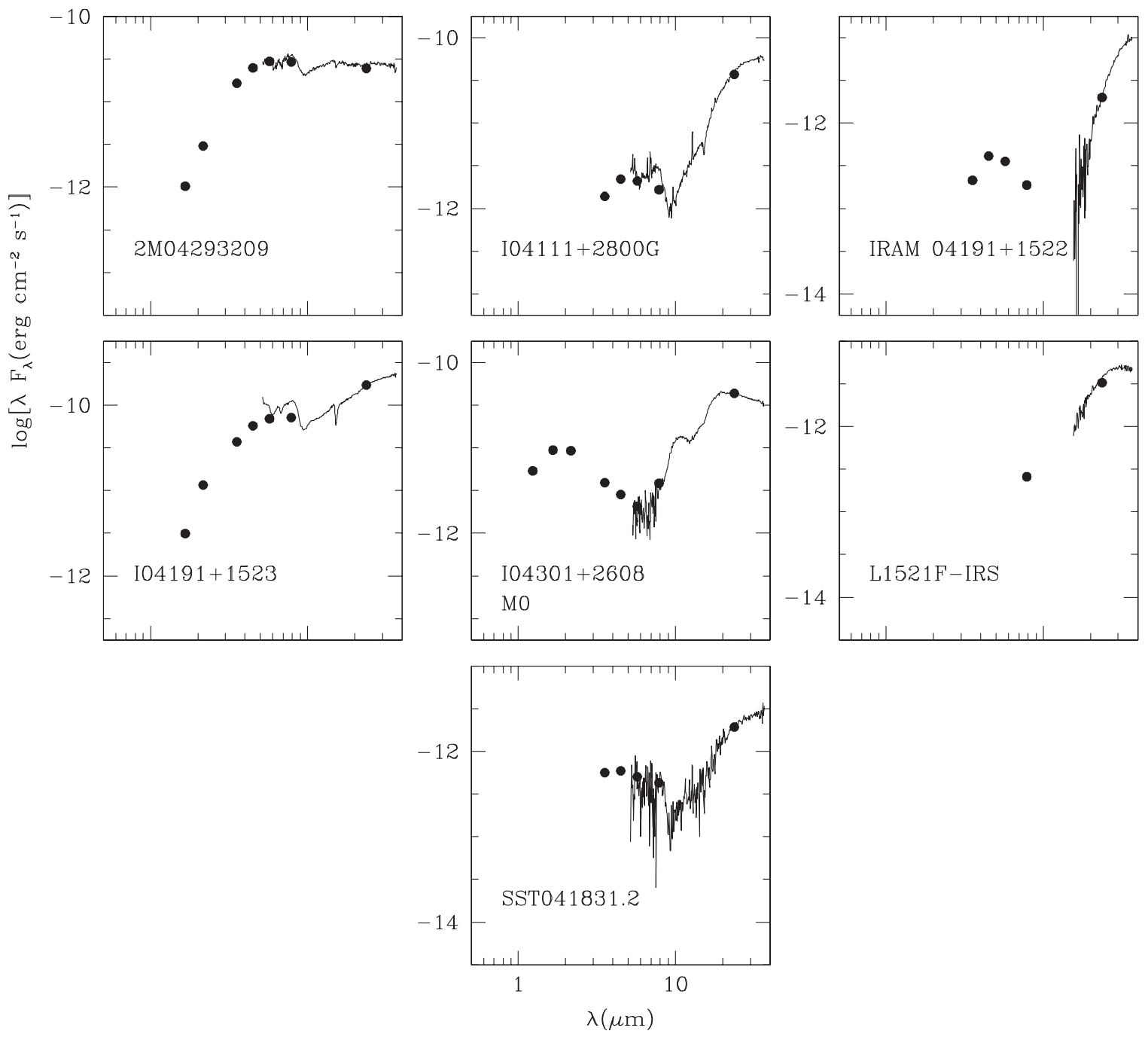

Figure 6. SEDs for the seven Class 0/I objects in Taurus presented in this work. The observed 2MASS, IRAC, and MIPS photometry is shown as black, filled circles, and the observed IRS spectrum as a solid line.

dwarf (its spectral type is M6) seems to have one of the most evolved disks in Taurus, with emission from the disk below levels expected from a flat (i.e., fully settled), optically thick disk and just a factor of $\sim 1.4-12$ above that of the photosphere over the entire IRS range. There are a few other objects with similar SEDs in Taurus, all with a spectral type around late $\mathrm{M}$ (see Luhman et al. 2010), but they are not part of our sample since they lack IRS data.

Four objects, 2MASS J04322415+2251083, CIDA 14, KPNO 13 , and MHO 5, have only $n_{13-31}$ values below $-4 / 3$, with $6-13 \mu \mathrm{m}$ indices in a typical range for accretion disks. They are all very low mass stars (spectral types range from M4.5 to M6) with small excesses below about $4.5 \mu \mathrm{m}$, but more typical amounts of infrared excesses at longer wavelengths. The steeply declining slope of the SED beyond $13 \mu \mathrm{m}$ could suggest truncated outer disks, as is probably the case for SR 20 in Ophiuchus (McClure et al. 2008), or flat disks with very low mass surface densities that are only moderately optically thick to optically thin (see Adame 2010; L. Adame et al. 2011, in preparation).

A spectral index closer to -3 , which is the value for blackbody emission in the Rayleigh-Jeans regime, suggests either emission dominated by a hot blackbody from an inner disk rim (for $n_{6-13}$ ), or emission by the stellar photosphere (both $n_{6-13}$ and $\left.n_{13-31}\right)$. For example, the $6-13 \mu \mathrm{m}$ emission of UX Tau A $\left(n_{6-13}=-1.96\right)$ is dominated by blackbody radiation from the inner disk wall (Espaillat et al. 2007a, 2010), while V819 Tau $\left(n_{6-13}=-2.56\right)$ has no excess emission out to about $8 \mu \mathrm{m}$ and thus its 6-13 spectral index reveals photospheric emission. As discussed in Section 4.5, two more objects, RX J0432.8+1735, and LkCa 19, have an SED similar to that of V819 Tau (LkCa 19 is not shown in Figure 7 since only the LH spectrum is available). These three objects could represent the most evolved protoplanetary disks in Taurus, when the dust has become optically thin and an inner hole is present.

While the 13-31 $\mu \mathrm{m}$ spectral index can be used as an indicator of dust settling, the strength of the $10 \mu \mathrm{m}$ silicate feature is a measure for the amount of optically thin dust emission. We calculated the equivalent width of the $10 \mu \mathrm{m}$ feature as in Furlan et al. (2009b) by subtracting and dividing each spectrum by the continuum defined as a polynomial anchored at 5.6-7.9, $13.0-14.0,14.5-15.5$, and $28.0-30.0 \mu \mathrm{m}$, then integrating the resulting spectrum from 8 to $13 \mu \mathrm{m}$. The $\operatorname{EW}(10 \mu \mathrm{m})$ values and the corresponding 13-31 $\mu \mathrm{m}$ spectral indices are plotted in Figure 8 (see also Table 5). The equivalent widths obtained here are in a few cases somewhat different from the ones in Furlan et al. (2009b), since here we derived new extinction values (which affect the shape of the dereddened $10 \mu \mathrm{m}$ feature). For 


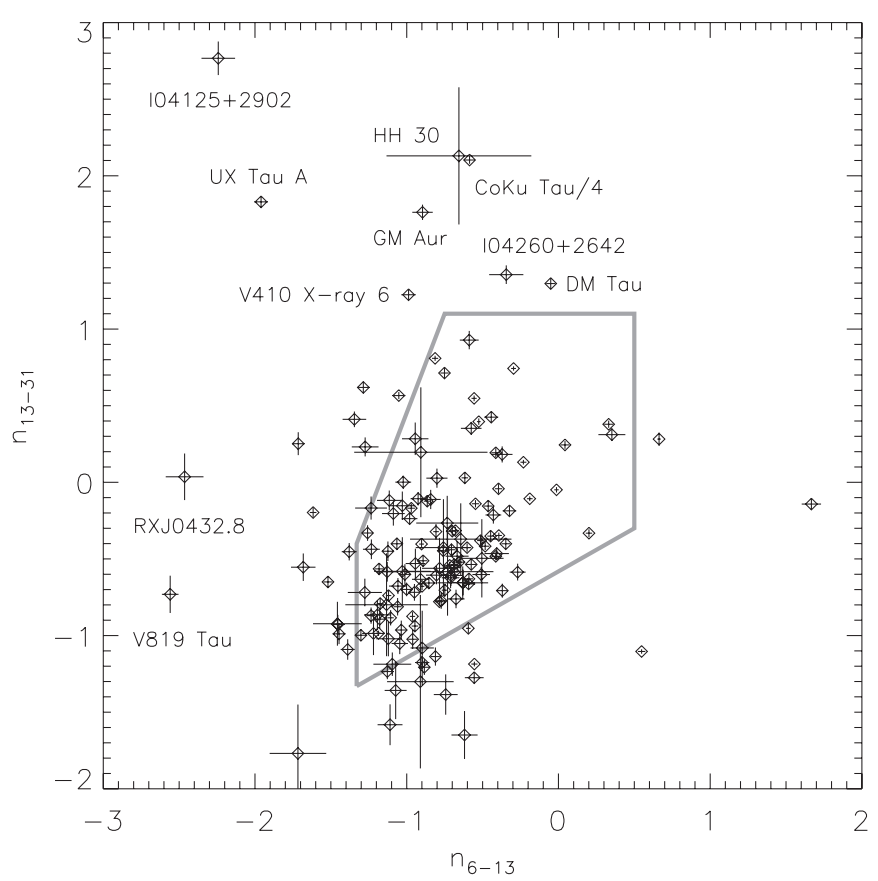

Figure 7. Spectral index between 13 and $31 \mu \mathrm{m}$ vs. the spectral index between 6 and $13 \mu \mathrm{m}$ for the T Tauri stars in our sample. The polygon delineates the region of typical optically thick accretion disks (with different degrees of dust settling) as derived from models of D'Alessio et al. (2006); the grid of models can be found in Espaillat (2009).

several objects, the uncertainties of the $\operatorname{EW}(10 \mu \mathrm{m})$ values are dominated by the uncertainties of the continuum fit and are therefore fairly large (e.g., MHO 3, Haro 6-13).

As can be seen from Figure 8, most objects lie in the region expected for full accretion disks (which is shown by the polygon); this region was adopted from Furlan et al. (2009b), where it was derived from the same grid of models as the one used earlier in this section. The $n_{13-31}$ outliers already identified in Figure 7 are labeled, as are some of the objects with unusually large $\mathrm{EW}(10 \mu \mathrm{m})$. This latter group encompasses the three most prominent $\mathrm{EW}(10 \mu \mathrm{m})$ outliers identified in Furlan et al. (2009b), LkCa 15, RY Tau, and MHO 3. LkCa 15 is a pre-transitional disk which is characterized by a large gap in the optically thick disk that is partially filled with optically thin dust (Piétu et al. 2006; Espaillat et al. 2007a, 2008, 2010, 2011; Thalmann et al. 2010). It is this dust, combined with the reduced continuum emission due to the gap in the disk, that generates the unusually strong equivalent width of the $10 \mu \mathrm{m}$ silicate emission feature (see also McClure et al. 2010; Manoj et al. 2011). In recent work, RY Tau was suggested to have a disk structure resembling that of $\mathrm{LkCa} 15$, with a gap about half as big and a smaller extent for the optically thin region (Isella et al. 2010; Espaillat et al. 2011). Also IP Tau, which is an $\mathrm{EW}(10 \mu \mathrm{m})$ outlier, could be modeled with a small gap within the optically thick disk filled with a very small amount of optically thin dust (Espaillat et al. 2011). Thus, it is possible that the other $\mathrm{EW}(10 \mu \mathrm{m})$ outliers have a similar disk structure.

We note that the equivalent width of the $10 \mu \mathrm{m}$ feature of UX Tau A also contains the contribution of a polycyclic aromatic hydrocarbon (PAH) feature at $11.3 \mu \mathrm{m}$; its $\mathrm{EW}(10 \mu \mathrm{m})$ value derived solely from the strength of its silicate emission would be about $0.1 \mu \mathrm{m}$ lower. Also the silicate features of AB Aur, SU Aur, and V892 Tau are affected by PAH emission at $\sim 8,8.6$ and $11.3 \mu \mathrm{m}$, but since their silicate features are quite strong, the contribution of the PAH emission to their $\mathrm{EW}(10 \mu \mathrm{m})$ values are very small.

Since about one-third of our sample consists of multiple systems, we checked whether there are any differences in the distribution of $n_{13-31}$ and $\operatorname{EW}(10 \mu \mathrm{m})$ values for the single and multiple systems in our sample (see Figure 9). The two histograms are very similar; two-sided Kolmogorov-Smirnov (K-S) tests (e.g., Press et al. 1993) indicate that the two data arrays, one for the single and one for the multiple stars, are drawn from the same distribution (K-S probability of 0.65 and 0.41 for $n_{13-31}$ and $\mathrm{EW}(10 \mu \mathrm{m})$, respectively). Even if we restrict the separation range of multiple systems to values less than a few tenths of an arcsecond (which corresponds to tens of $\mathrm{AU}$ ) or to a few arcseconds (which corresponds to a few to several hundred AU), there is no significant difference in the

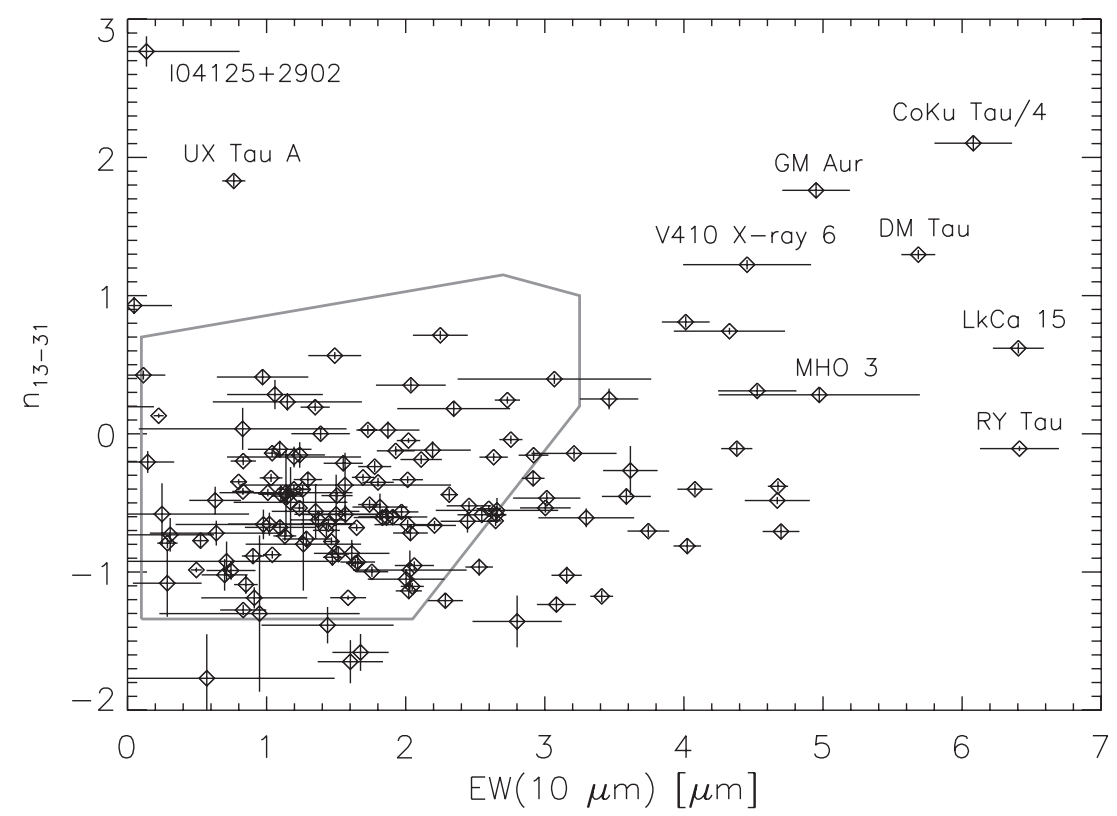

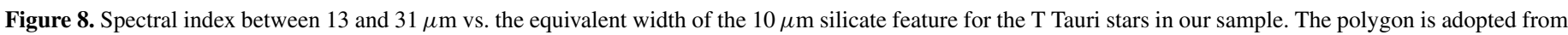
Furlan et al. (2009b) as the region occupied by optically thick accretion disks with different degrees of dust settling. 
Table 5

Spectral Indices and Silicate Feature Indices for the T Tauri Stars in Taurus with Infrared Excesses Observed by IRS

\begin{tabular}{|c|c|c|c|c|c|}
\hline $\begin{array}{l}\text { Name } \\
\text { (1) }\end{array}$ & $\begin{array}{l}n_{6-13} \\
(2)\end{array}$ & $\begin{array}{c}n_{13-31} \\
(3)\end{array}$ & $\begin{array}{c}\mathrm{EW}(10 \mu \mathrm{m}) \\
(4)\end{array}$ & $\begin{array}{l}\text { Peak-over-continuum } \\
\text { (5) }\end{array}$ & $\begin{array}{c}F_{11.3} / F_{9.8} \\
\quad(6)\end{array}$ \\
\hline 2MASS J04141188+2811535 & -0.51 & -0.60 & 1.91 & 1.58 & 0.94 \\
\hline 2MASS J04153916+2818586 & -1.35 & 0.41 & 0.97 & 1.30 & 0.89 \\
\hline 2MASS J04155799+2746175 & -1.05 & -1.05 & 2.00 & 1.65 & 0.94 \\
\hline 2MASS J04163911+2858491 & -1.46 & -0.92 & 0.71 & 1.27 & 0.95 \\
\hline 2MASS J04201611+2821325 & -0.90 & -1.08 & 0.29 & 1.17 & 0.93 \\
\hline 2MASS J04202144+2813491 & -0.91 & 0.20 & -0.78 & 0.89 & 1.75 \\
\hline 2MASS J04202606+2804089 & 0.35 & 0.31 & 4.53 & 2.00 & 0.87 \\
\hline 2MASS J04214631+2659296 & -0.73 & -0.58 & 1.50 & 1.51 & 0.94 \\
\hline 2MASS J04230607+2801194 & -1.03 & -0.15 & 1.24 & 1.38 & 0.91 \\
\hline 2MASS J04242090+2630511 & -0.67 & -0.48 & 0.63 & 1.20 & 0.91 \\
\hline 2MASS J04242646+2649503 & -0.71 & -0.62 & 1.37 & 1.34 & 0.99 \\
\hline 2MASS J04263055+2443558 & -0.91 & -1.30 & 0.95 & 1.37 & 0.85 \\
\hline 2MASS J04284263+2714039 & -1.68 & -0.55 & 2.66 & 1.77 & 1.02 \\
\hline 2MASS J04290068+2755033 & -0.78 & -0.56 & 1.35 & 1.38 & 0.94 \\
\hline 2MASS J04295950+2433078 & -1.13 & -0.58 & 1.57 & 1.53 & 0.88 \\
\hline 2MASS J04322415+2251083 & -0.74 & -1.38 & 1.44 & 1.48 & 0.92 \\
\hline 2MASS J04330945+2246487 & -1.72 & -1.77 & 0.57 & 1.23 & 1.03 \\
\hline 2MASS J04333905+2227207 & -0.59 & 0.93 & 0.05 & 0.76 & 1.25 \\
\hline 2MASS J04334465+2615005 & -1.06 & -0.68 & 1.10 & 1.33 & 0.95 \\
\hline 2MASS J04335245+2612548 & -0.50 & -0.50 & 1.17 & 1.33 & 0.90 \\
\hline 2MASS J04361030+2159364 & -0.76 & -0.43 & 1.14 & 1.35 & 0.95 \\
\hline 2MASS J04381486+2611399 & -0.44 & 0.43 & 0.11 & 0.89 & 1.21 \\
\hline 2MASS J04390163+2336029 & -1.22 & -0.99 & 2.03 & 1.69 & 0.93 \\
\hline 2MASS J04390396+2544264 & -0.68 & -0.76 & 1.29 & 1.35 & 0.91 \\
\hline 2MASS J04393364+2359212 & -0.71 & -0.61 & 3.30 & 2.13 & 0.79 \\
\hline 2MASS J04400067+2358211 & -0.94 & 0.28 & 1.06 & 1.29 & 0.96 \\
\hline 2MASS J04414489+2301513 & -1.03 & -0.58 & 0.25 & 1.05 & 1.02 \\
\hline 2MASS J04414825+2534304 & $\ldots$ & $\ldots$ & $\ldots$ & 1.48 & 0.92 \\
\hline 2MASS J04442713+2512164 & -0.35 & -0.40 & 1.20 & 1.33 & 0.95 \\
\hline 2MASS J04554535+3019389 & -1.12 & -1.02 & 0.70 & 1.22 & 1.00 \\
\hline 2MASS J04554801+3028050 & -0.63 & -0.65 & 1.02 & 1.26 & 0.92 \\
\hline AA Tau & -0.89 & -0.51 & 1.74 & 1.60 & 0.86 \\
\hline AB Aur & -0.81 & 0.81 & 4.01 & 2.59 & 0.77 \\
\hline BP Tau & -0.69 & -0.58 & 2.65 & 1.89 & 0.82 \\
\hline CIDA 1 & -0.27 & -0.59 & 2.55 & 1.95 & 0.75 \\
\hline CIDA 7 & -0.57 & 0.35 & 2.04 & 1.63 & 0.90 \\
\hline CIDA 9 & -0.85 & -0.66 & 2.01 & 1.62 & 0.93 \\
\hline CIDA 11 & -1.10 & -1.19 & 0.91 & 1.32 & 0.98 \\
\hline CIDA 12 & -0.63 & -0.66 & 0.98 & 1.25 & 0.98 \\
\hline CIDA 14 & -1.07 & -1.36 & 2.80 & 1.85 & 0.90 \\
\hline CI Tau & -0.97 & -0.17 & 2.63 & 1.86 & 0.88 \\
\hline CoKu Tau/3 & -1.13 & -1.24 & 3.08 & 2.17 & 0.82 \\
\hline $\mathrm{CoKu} \mathrm{Tau} / 4$ & -0.59 & 2.10 & 6.08 & 1.94 & 0.96 \\
\hline CW Tau & -1.12 & -0.74 & 1.13 & 1.34 & 0.94 \\
\hline CX Tau & -0.32 & -0.19 & 2.11 & 1.55 & 0.88 \\
\hline CY Tau & -1.45 & -0.99 & 0.74 & 1.27 & 0.97 \\
\hline CZ Tau & 0.55 & -1.10 & 2.05 & 1.53 & 0.86 \\
\hline DD Tau & -0.79 & -0.78 & 1.47 & 1.49 & 0.88 \\
\hline DE Tau & -0.87 & -0.12 & 1.93 & 1.57 & 0.91 \\
\hline DF Tau & -1.39 & -1.09 & 0.85 & 1.34 & 0.92 \\
\hline DG Tau & -0.23 & 0.13 & 0.22 & 1.01 & 1.01 \\
\hline DH Tau & -1.72 & 0.25 & 3.46 & 1.96 & 1.00 \\
\hline DK Tau & -1.06 & -0.81 & 4.02 & 2.15 & 0.91 \\
\hline DL Tau & -0.77 & -0.77 & 0.52 & 1.15 & 1.00 \\
\hline DM Tau & -0.05 & 1.30 & 5.68 & 1.94 & 1.04 \\
\hline DN Tau & -0.60 & -0.43 & 1.01 & 1.24 & 0.96 \\
\hline DO Tau & -0.55 & -0.14 & 1.04 & 1.27 & 0.97 \\
\hline DP Tau & -0.45 & -0.35 & 1.80 & 1.59 & 0.88 \\
\hline DQ Tau & -0.48 & -0.42 & 0.83 & 1.24 & 0.94 \\
\hline DR Tau & -0.90 & -0.40 & 1.26 & 1.43 & 0.88 \\
\hline DS Tau & -1.04 & -0.96 & 2.53 & 1.85 & 0.85 \\
\hline FM Tau & -0.46 & -0.16 & 2.92 & 1.91 & 0.81 \\
\hline FN Tau & 0.20 & -0.33 & 2.01 & 1.47 & 0.94 \\
\hline FO Tau & -0.68 & -0.31 & 1.69 & 1.47 & 0.91 \\
\hline
\end{tabular}


Table 5

(Continued)

\begin{tabular}{|c|c|c|c|c|c|}
\hline $\begin{array}{l}\text { Name } \\
\text { (1) }\end{array}$ & $\begin{array}{c}n_{6-13} \\
(2)\end{array}$ & $\begin{array}{c}n_{13-31} \\
(3)\end{array}$ & $\begin{array}{c}\mathrm{EW}(10 \mu \mathrm{m}) \\
\quad(4)\end{array}$ & $\begin{array}{l}\text { Peak-over-continuum } \\
\text { (5) }\end{array}$ & $\begin{array}{l}F_{11.3} / F_{9.8} \\
\quad(6)\end{array}$ \\
\hline FP Tau & -0.84 & -0.11 & 1.09 & 1.33 & 0.88 \\
\hline FQ Tau & -1.12 & -0.45 & 1.15 & 1.34 & 0.95 \\
\hline FR Tau & -0.56 & -1.27 & 0.83 & 1.28 & 0.93 \\
\hline FS Tau & -0.62 & 0.03 & 1.73 & 1.56 & 0.85 \\
\hline FT Tau & -0.65 & -0.52 & 2.45 & 1.79 & 0.85 \\
\hline FV Tau & -0.90 & -0.68 & 1.65 & 1.59 & 0.87 \\
\hline FV Tau/c & $\ldots$ & $\ldots$ & $\ldots$ & 1.42 & 0.91 \\
\hline FX Tau & -1.07 & -0.40 & 4.08 & 2.37 & 0.82 \\
\hline FY Tau & -1.52 & -0.65 & 1.44 & 1.47 & 0.90 \\
\hline FZ Tau & -1.18 & -0.89 & 1.47 & 1.43 & 0.94 \\
\hline GG Tau & -0.81 & -0.32 & 2.92 & 1.98 & 0.82 \\
\hline GH Tau & -1.26 & -0.33 & 1.30 & 1.38 & 0.95 \\
\hline GI Tau & -0.59 & -0.63 & 2.65 & 1.92 & 0.81 \\
\hline [GKH94] 41 & -0.37 & 0.18 & 2.35 & 1.60 & 0.82 \\
\hline GK Tau & -0.51 & -0.38 & 4.68 & 2.52 & 0.80 \\
\hline GM Aur & -0.90 & 1.76 & 4.95 & 2.85 & 0.74 \\
\hline GM Tau & -1.19 & -0.87 & 1.61 & 1.61 & 0.85 \\
\hline GN Tau & -0.96 & -1.02 & 3.16 & 1.95 & 0.89 \\
\hline GO Tau & -0.80 & 0.03 & 1.87 & 1.61 & 0.89 \\
\hline Haro6-5B & 0.33 & 0.38 & -1.36 & 0.45 & 1.29 \\
\hline Haro6-13 & -0.52 & 0.40 & 3.07 & 1.65 & 0.84 \\
\hline Haro6-28 & -0.94 & -0.53 & 1.82 & 1.56 & 0.91 \\
\hline Haro6-37 & -1.24 & -0.87 & 1.51 & 1.52 & 0.88 \\
\hline HH 30 & -0.66 & 2.13 & -1.01 & 0.74 & 0.81 \\
\hline HK Tau & -0.75 & 0.71 & 2.25 & 1.53 & 0.96 \\
\hline HN Tau & -0.70 & -0.44 & 2.31 & 1.74 & 0.87 \\
\hline HO Tau & -0.91 & -0.63 & 2.44 & 1.76 & 0.87 \\
\hline HP Tau & -0.40 & -0.04 & 2.76 & 1.89 & 0.80 \\
\hline HQ Tau & -0.75 & -0.70 & 3.74 & 2.41 & 0.72 \\
\hline IC 2087 IR & -1.19 & -0.99 & 0.50 & 1.22 & 0.89 \\
\hline IRAS 04108+2910 & -1.17 & -0.79 & 0.29 & 1.10 & 1.04 \\
\hline IRAS $04125+2902$ & -2.24 & 2.77 & 0.14 & 1.11 & 0.96 \\
\hline IRAS $04154+2823$ & -0.70 & -0.32 & 1.03 & 1.33 & 0.87 \\
\hline IRAS $04173+2812$ & -0.59 & -0.66 & 2.21 & 1.75 & 0.84 \\
\hline IRAS 04187+1927 & -0.59 & -0.95 & 2.06 & 1.71 & 0.82 \\
\hline IRAS F04192+2647 & -1.18 & -0.56 & 1.97 & 1.65 & 0.88 \\
\hline IRAS 04196+2638 & -0.72 & -0.54 & 2.60 & 1.86 & 0.82 \\
\hline IRAS $04200+2759$ & -0.57 & -0.54 & 3.00 & 2.13 & 0.75 \\
\hline IRAS $04216+2603$ & -0.76 & -0.45 & 1.51 & 1.56 & 0.84 \\
\hline IRAS $04260+2642$ & -0.34 & 1.36 & -0.71 & 0.65 & 1.46 \\
\hline IRAS $04263+2654$ & -0.98 & -0.24 & 1.78 & 1.56 & 0.87 \\
\hline IRAS $04278+2253$ & -0.55 & -1.19 & 1.59 & 1.49 & 0.85 \\
\hline IRAS 04303+2240 & -0.81 & -1.14 & 2.02 & 1.67 & 0.88 \\
\hline IRAS $04370+2559$ & -0.37 & -0.71 & 4.70 & 2.64 & 0.69 \\
\hline IRAS $04385+2550$ & 0.04 & 0.24 & 2.73 & 1.88 & 0.78 \\
\hline IP Tau & -0.93 & -0.11 & 4.38 & 2.57 & 0.74 \\
\hline IQ Tau & -1.30 & -1.00 & 1.76 & 1.58 & 0.93 \\
\hline IS Tau & -0.88 & -1.21 & 2.29 & 1.62 & 0.98 \\
\hline IT Tau & -1.45 & -0.93 & 1.65 & 1.62 & 0.84 \\
\hline ITG 1 & -0.73 & -0.27 & 3.62 & 2.14 & 0.94 \\
\hline ITG 15 & -1.02 & 0.00 & 1.39 & 1.47 & 0.86 \\
\hline ITG 33A & -0.41 & -0.47 & 3.01 & 1.93 & 0.85 \\
\hline ITG 34 & -0.80 & -0.61 & 1.83 & 1.60 & 0.83 \\
\hline ITG 40 & -1.24 & -0.17 & 1.20 & 1.24 & 0.98 \\
\hline JH 112 A & -0.41 & -0.48 & 4.67 & 2.39 & 0.90 \\
\hline JH 112 B & $\ldots$ & $\ldots$ & $\ldots$ & 1.70 & 0.89 \\
\hline JH 223 & -1.28 & -0.72 & 0.64 & 1.30 & 0.90 \\
\hline KPNO 3 & -0.64 & -0.37 & 1.56 & 1.41 & 0.93 \\
\hline KPNO 7 & -1.13 & -0.80 & 1.26 & 1.48 & 0.95 \\
\hline KPNO 10 & -0.43 & -0.21 & 1.55 & 1.45 & 0.96 \\
\hline KPNO 13 & -1.11 & -1.58 & 1.67 & 1.59 & 0.76 \\
\hline LkCa 15 & -1.29 & 0.62 & 6.40 & 3.50 & 0.66 \\
\hline LkHa 358 & -1.05 & 0.57 & 1.49 & 1.51 & 0.95 \\
\hline LR 1 & -1.27 & 0.23 & 1.15 & 1.43 & 0.82 \\
\hline MHO 3 & 0.66 & 0.28 & 4.97 & 2.41 & 0.82 \\
\hline
\end{tabular}


Table 5

(Continued)

\begin{tabular}{|c|c|c|c|c|c|}
\hline $\begin{array}{l}\text { Name } \\
\text { (1) }\end{array}$ & $\begin{array}{l}n_{6-13} \\
(2)\end{array}$ & $\begin{array}{c}n_{13-31} \\
(3)\end{array}$ & $\begin{array}{c}\mathrm{EW}(10 \mu \mathrm{m}) \\
\text { (4) }\end{array}$ & $\begin{array}{l}\text { Peak-over-continuum } \\
\text { (5) }\end{array}$ & $\begin{array}{l}F_{11.3} / F_{9.8} \\
\text { (6) }\end{array}$ \\
\hline MHO 5 & -0.62 & -1.65 & 1.60 & 1.44 & 0.97 \\
\hline MHO 6 & -1.09 & -0.20 & 0.14 & 1.05 & 0.98 \\
\hline RW Aur & -0.69 & -0.54 & 1.24 & 1.44 & 0.87 \\
\hline RX J0432.8+1735 & -2.46 & 0.04 & 0.83 & 1.23 & 0.84 \\
\hline RY Tau & -0.19 & -0.11 & 6.41 & 3.24 & 0.72 \\
\hline SU Aur & -0.30 & 0.74 & 4.33 & 2.43 & 0.84 \\
\hline T Tau & -0.56 & 0.55 & -0.31 & 0.90 & 1.07 \\
\hline UX Tau A+C & -1.96 & 1.83 & 0.76 & 1.20 & 1.13 \\
\hline UY Aur & -0.01 & -0.05 & 2.02 & 1.65 & 0.81 \\
\hline UZ Tau A & -0.95 & -0.72 & 2.03 & 1.76 & 0.86 \\
\hline V410 Anon 13 & -1.01 & -0.60 & 1.86 & 1.61 & 0.90 \\
\hline V410 X-ray 1 & -0.90 & -1.18 & 3.41 & 2.30 & 0.77 \\
\hline V410 X-ray 6 & -0.99 & 1.22 & 4.45 & 2.07 & 0.88 \\
\hline V710 Tau & -1.00 & -0.70 & 1.43 & 1.49 & 0.91 \\
\hline V773 Tau & -0.96 & -0.88 & 1.04 & 1.28 & 0.98 \\
\hline V807 Tau & -1.62 & -0.20 & 0.83 & 1.28 & 0.96 \\
\hline V819 Tau & -2.56 & -0.73 & 0.31 & 1.20 & 0.95 \\
\hline V836 Tau & -1.38 & -0.45 & 3.59 & 2.14 & 0.86 \\
\hline V892 Tau & 1.67 & -0.14 & 3.21 & 1.86 & 0.96 \\
\hline V955 Tau & -0.95 & -0.94 & 1.64 & 1.47 & 0.93 \\
\hline VY Tau & -1.12 & -0.12 & 2.19 & 1.66 & 0.94 \\
\hline XEST 13-010 & -1.23 & -0.44 & 1.10 & 1.38 & 0.88 \\
\hline XZ Tau & -0.39 & -0.35 & 0.80 & 1.21 & 0.94 \\
\hline ZZ Tau & -1.11 & -0.88 & 0.90 & 1.22 & 0.93 \\
\hline ZZ Tau IRS & -0.41 & 0.19 & 1.35 & 1.31 & 1.02 \\
\hline
\end{tabular}

Notes. Column 1 lists the name of the object, Column 2 lists the $6-13 \mu \mathrm{m}$ spectral index, Column 3 lists the $13-31 \mu \mathrm{m}$ spectral index, Column 4 lists the equivalent width of the $10 \mu \mathrm{m}$ silicate emission feature (in $\mu \mathrm{m}$ ), Column 5 lists the peak-over-continuum ratio of the $10 \mu \mathrm{m}$ feature, and Column 6 lists the 11.3 and $9.8 \mu \mathrm{m}$ flux ratio.

distribution of $n_{13-31}$ and EW(10 $\left.\mu \mathrm{m}\right)$ values. This confirms the results of Pascucci et al. (2008) who analyzed the IRS spectra of a subset (44 objects) of the Taurus sample. Therefore, the disk structure for objects in multiple systems is on average very similar to that of single objects. However, since the multiplicity information for our targets is likely not complete, especially for fainter objects and at closer separations $(\lesssim 15 \mathrm{AU}$; see also Section 3.2), we might miss the influence that close binaries can have on disk structure and evolution (see Jensen et al. 1994; Jensen \& Mathieu 1997; Bouwman et al. 2006).

Figures 10 and 11 show the $\operatorname{EW}(10 \mu \mathrm{m})$ and $n_{13-31}$ values, respectively, for each spectral type bin. There seems to be a marginally significant trend of lower $13-31 \mu \mathrm{m}$ spectral indices for later spectral types, while the trend for the $10 \mu \mathrm{m}$ equivalent widths is not statistically significant. However, one has to keep in mind that the stellar photospheres become redder for later spectral types, so the actual slope of the excess is somewhat steeper for these late-type stars (see Luhman et al. 2010 and Section 8). We note that none of the objects with spectral types ranging from M6 to M9 (the very low mass stars and brown dwarfs) are $\mathrm{EW}(10 \mu \mathrm{m})$ or $n_{13-31}$ outliers when compared to our model grid, which applies to low-mass stars. Models of brown dwarf accretion disks occupy a smaller area in EW $(10 \mu \mathrm{m})-n_{13-31}$ space; they typically do not result in objects with $\mathrm{EW}(10 \mu \mathrm{m})$ values larger than about $2 \mu \mathrm{m}$ and positive 13-31 $\mu \mathrm{m}$ spectral indices (Adame 2010; L. Adame et al. 2011, in preparation). Therefore, [GKH94] 41, with a spectral type of $\mathrm{M} 7.5, \mathrm{EW}(10 \mu \mathrm{m})=2.35 \mu \mathrm{m}$ and $n_{13-31}=0.18$ could be an outlier. The clearest outlier with the latest spectral type is V410 X-ray 6 (M5.5). Not considering the two edge-on disks HH 30 and IRAS 04260+2642, which have large $n_{13-31}$ values, but negative $\operatorname{EW}(10 \mu \mathrm{m})$, most $n_{13-31}$ outliers have a spectral type of M1 (but we are dealing with very small numbers). The largest number of $\mathrm{EW}(10 \mu \mathrm{m})$ outliers can be found among objects with spectral types K7 and M1; some of these objects are also $n_{13-31}$ outliers.

The fraction of objects whose $10 \mu \mathrm{m}$ feature is unusually strong, but whose $n_{13-31}$ value lies in the normal range, amounts to $18 \% \pm 3 \%$, basically the same (within $1 \sigma$ uncertainty) to what we found in Furlan et al. (2009b), but with a sample that is $50 \%$ larger. The fraction of transitional disks, based solely on the $13-31 \mu \mathrm{m}$ spectral index, is $4.0 \% \pm 1.6 \%$ (or $3.3 \% \pm 1.5 \%$, if $\mathrm{CoKu} \mathrm{Tau} / 4$, which is actually a circumbinary disk, is left out), again very similar to the result from Furlan et al. (2009b).

Of all the objects with $\mathrm{EW}(10 \mu \mathrm{m})$ values larger than expected for continuous accretion disks, $13 \% \pm 6 \%$ are also outliers in $n_{13-31}$ (with values for the spectral index $\gtrsim 1$ ). The median $13-31 \mu \mathrm{m}$ spectral index for the other $\operatorname{EW}(10 \mu \mathrm{m})$ outliers amounts to -0.45 , which is a value indicative of highly settled disks (dust depletion factors in the upper layers by at least a factor of 1000) and comparable to the median $n_{13-31}$ value of -0.48 for the whole sample. Thus, most $\operatorname{EW}(10 \mu \mathrm{m})$ outliers are fairly typical in terms of dust settling in their disks despite the likely presence of gaps.

\section{PROPERTIES OF THE DUST}

In order to study the degree of dust processing, we computed the quantities for the "shape versus strength" diagram, which measures the properties of the $10 \mu \mathrm{m}$ silicate emission feature (van Boekel et al. 2003): the shape is represented by the flux ratio at 11.3 and $9.8 \mu \mathrm{m}\left(F_{11.3} / F_{9.8}\right)$ of the continuum-subtracted and 

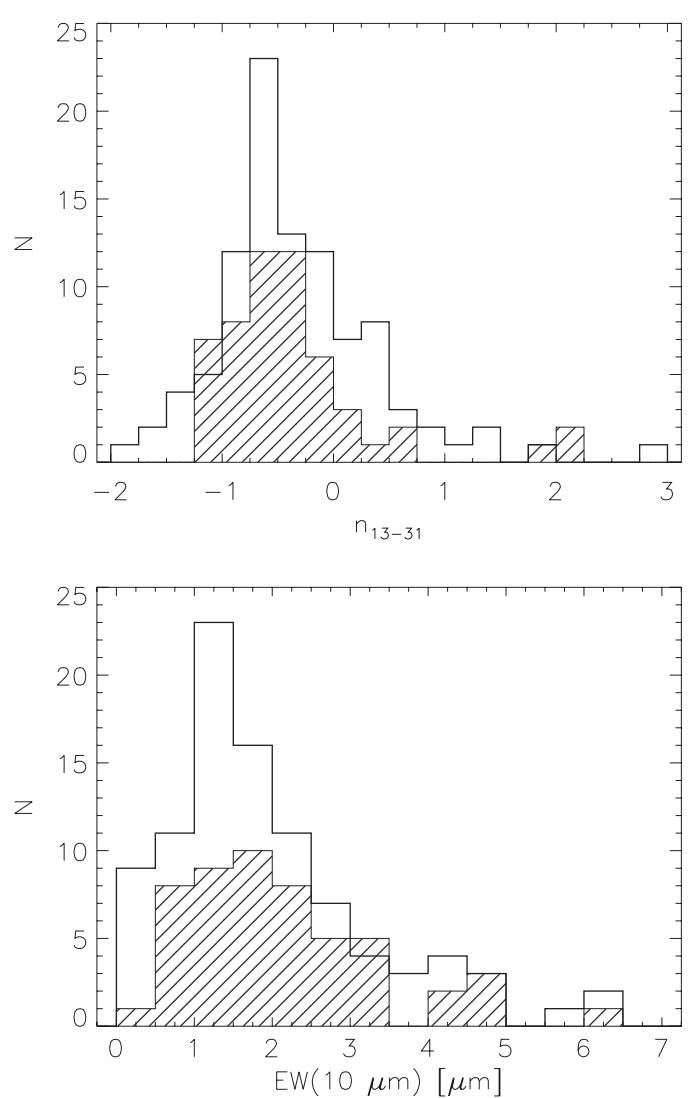

Figure 9. Histograms for the spectral index between 13 and $31 \mu \mathrm{m}$ and the equivalent width of the $10 \mu \mathrm{m}$ silicate feature for the single T Tauri stars in our sample (white area) and for multiple systems (shaded area).

continuum-normalized spectrum, while the strength is the ratio of the flux at $9.7 \mu \mathrm{m}$ and the average of the continuum flux in the $8-13 \mu \mathrm{m}$ wavelength region (the strength is also known as the "peak-over-continuum" ratio). To create the continuumsubtracted and continuum-normalized spectrum, we subtracted the continuum fit from the observed spectrum, divided by the average continuum flux in the $8-13 \mu \mathrm{m}$ region, and added 1.0. In this spectrum, the fluxes at 11.3 and $9.8 \mu \mathrm{m}$ were determined by averaging fluxes within $\pm 0.1 \mu \mathrm{m}$ of these wavelengths.

A larger value of $F_{11.3} / F_{9.8}$ suggests that the grains are processed, either via grain growth or crystallization, resulting in a flat $10 \mu \mathrm{m}$ feature (e.g., Olofsson et al. 2009). The crystalline dust species enstatite and forsterite have strong features at 11.1 and $11.2 \mu \mathrm{m}$, respectively (Jäger et al. 1998), while amorphous silicate grains display a broader feature peaked at $9.8 \mu \mathrm{m}$ that becomes wider for larger grains (Dorschner et al. 1995). PAHs also have a feature at $11.3 \mu \mathrm{m}$, but since we usually do not detect the other shorter-wavelength PAH features (at 6.2, 7.7, and $8.6 \mu \mathrm{m}$ ) in our spectra of low-mass stars, we can exclude PAHs as contributors to the $11.3 \mu \mathrm{m}$ flux of these objects.

Agreeing with results of several authors (e.g., van Boekel et al. 2005; Kessler-Silacci et al. 2006; Bouwman et al. 2008; Juhász et al. 2010), we find that the degree of dust processing decreases with the strength of the feature (see Figure 12; data are listed in Table 5). Moreover, very low mass objects and brown dwarfs (spectral types M6-M9) have weak silicate features and highly processed dust, which also agrees with previous findings (Apai et al. 2005; Pascucci et al. 2009; Riaz 2009). Figures 13 and 14 illustrate these trends of decreasing peak-over-continuum ratios and increasing $F_{11.3} / F_{9.8}$ values for later spectral types.

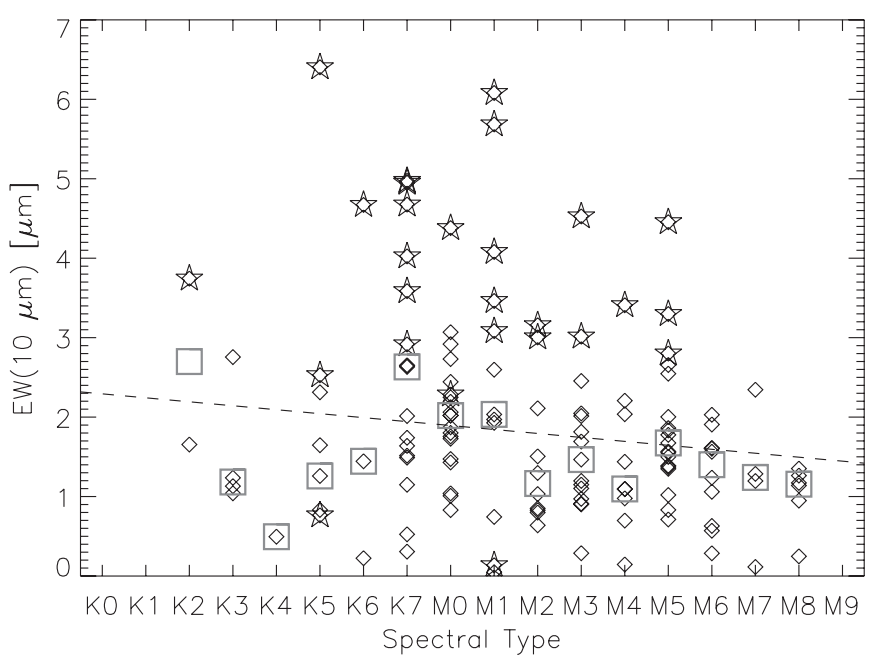

Figure 10. Equivalent width of the $10 \mu \mathrm{m}$ silicate feature vs. the spectral type for the T Tauri stars in our sample. The "outliers" from Figure 8 (those objects that cannot be described by full accretion disks; see the text) are shown with star symbols. For each spectral type, a square is plotted at the median $\operatorname{EW}(10 \mu \mathrm{m})$ value. The dashed line represents a linear fit to the data shown.

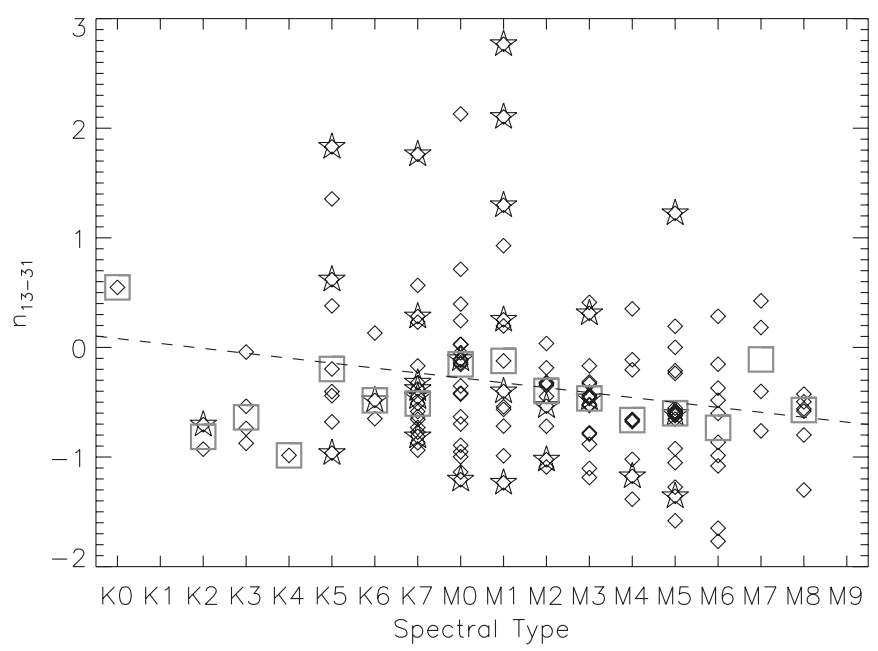

Figure 11. Spectral index between 13 and $31 \mu \mathrm{m}$ vs. the spectral type for the $\mathrm{T}$ Tauri stars in our sample. The star symbols have the same meaning as in Figure 10. Squares are plotted at the median $n_{13-31}$ value for each spectral type. The dashed line represents a linear fit to the data shown.

These figures also show the outliers identified in Section 5; since most of them are objects with unusually large $\mathrm{EW}(10 \mu \mathrm{m})$ values, their peak-over-continuum ratios are typically high, too. Figure 14 reveals that the degree of grain processing in the disks of outliers can vary substantially, in particular around stars with spectral types from K5 to M1; for the outliers among the later spectral types, the dust grains seem to be somewhat more pristine.

To see whether multiplicity has an effect on the strength and shape of the silicate feature, in Figure 15 we plotted the distributions of the peak-over-continuum ratios and of the $F_{11.3} /$ $F_{9.8}$ values for the single and multiple systems in our sample. As found for the $n_{13-31}$ and $\mathrm{EW}(10 \mu \mathrm{m})$ values, two-sided $\mathrm{K}-\mathrm{S}$ tests suggest that the data arrays for the single and multiple stars are drawn from the same distribution (K-S probability of 0.43 and 0.92 for the peak-over-continuum and $F_{11.3} / F_{9.8}$ ratio, respectively). This is in agreement with the results of Pascucci et al. (2008) who used a smaller sample of IRS spectra of $\mathrm{T}$ Tauri stars in Taurus. Setting upper limits on the separations of 


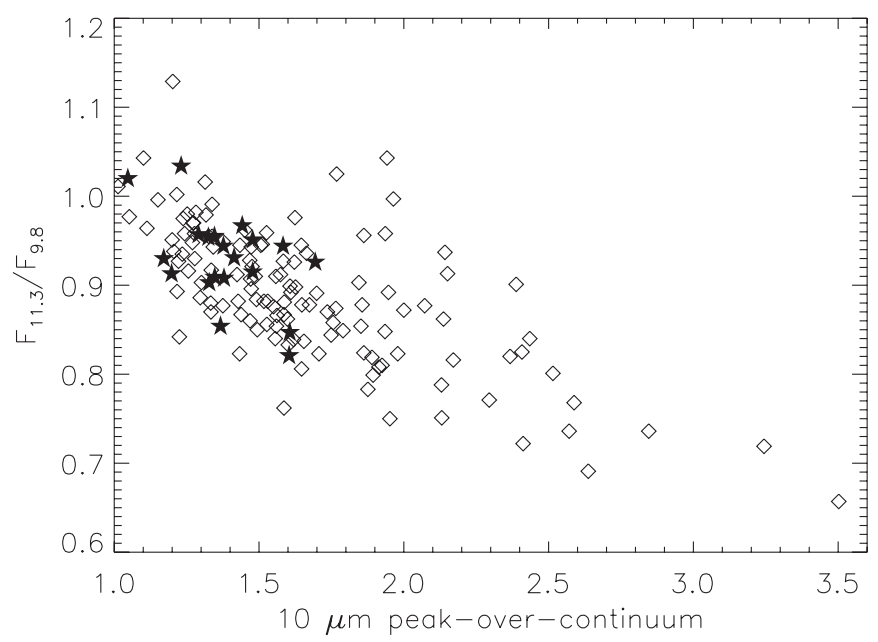

Figure 12. Shape vs. strength of the $10 \mu \mathrm{m}$ silicate feature for all Class II objects in our Taurus sample: the flux ratio of the continuum-subtracted and continuum-normalized spectrum at 11.3 and $9.8 \mu \mathrm{m}$ is plotted vs. the ratio of the peak flux around $9.7 \mu \mathrm{m}$ and the underlying continuum (see the text for details). The star symbol denotes objects with spectral type M6 or later.

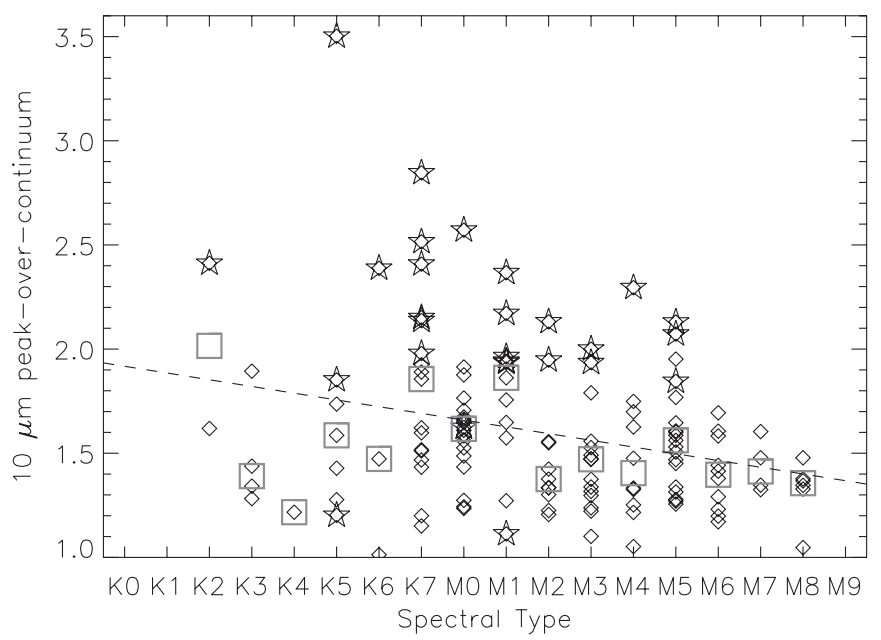

Figure 13. Peak-over-continuum ratio of the $10 \mu \mathrm{m}$ silicate feature vs. the spectral type for the T Tauri stars in our sample. The "outliers" from Figure 8 (see Section 5) are shown with star symbols. For each spectral type, a square is plotted at the median peak-over-continuum value. The dashed line represents a linear fit to the data shown.

components in multiple systems does not affect this conclusion. Thus, dust growth and processing is not influenced by the number of stars in a system, but very close binaries, whose census is likely not complete in our sample (see Section 3.2), probably have an effect on the dust in the inner disk.

In a recent work, Riaz (2009) studied the $10 \mu \mathrm{m}$ silicate feature in brown dwarf disks, using some of the same IRS data presented here, but reduced independently. The author found that most brown dwarf disks in Taurus are dominated by processed dust, i.e., they have relatively high large- and crystalline-grain mass fractions. While we qualitatively agree with this result, we point out that several of the spectra presented in Riaz (2009) that are also part of our sample appear different in our reductions. This could point to background-subtraction, extraction, or calibration issues in the reduction of their spectra, and to differences in the definition of the underlying continuum. In particular, it seems that 2MASS J04335245+2612548, KPNO 3 , and KPNO 7 have weak, but clearly detected silicate emission features in our versions of the reduced spectra (see Figure 16),

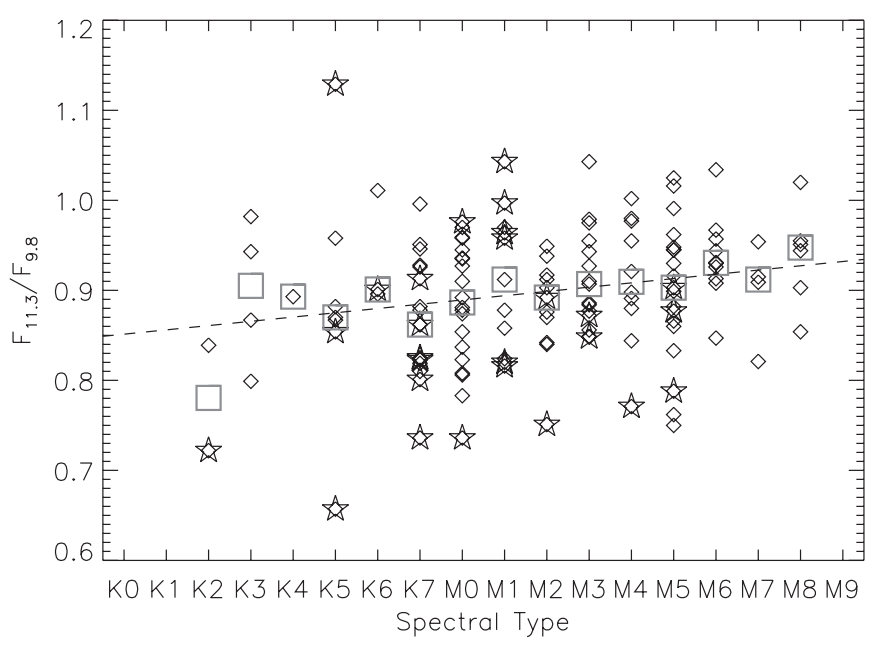

Figure 14. 11.3 and $9.8 \mu \mathrm{m}$ flux ratio vs. the spectral type for the T Tauri stars in our sample. The star symbols have the same meaning as in Figure 13. Squares are plotted at the median $F_{11.3} / F_{9.8}$ value for each spectral type. The dashed line represents a linear fit to the data shown.

while in Riaz (2009) there appears to be no feature. Most silicate emission features of very low mass stars and brown dwarfs appear stronger in our spectra than in the ones presented by Riaz (2009), in particular for 2MASS J04230607+2801194, 2MASS J04295950+2433078 (also known as CFHT-BD-Tau 20), and 2MASS J04554801+3028050. In KPNO 6, a very weak silicate feature could be present, both in our data and those of Riaz (2009), but since it was observed only with SL1, there is little continuum measured beyond $\sim 13 \mu \mathrm{m}$ to allow a reliable determination of the silicate feature strength. This difficulty in estimating the continuum level also applies to KPNO 12 (not included in the sample of Riaz 2009); however, KPNO 12 most likely lacks a silicate feature. We note that MHO 4, whose IRS spectrum is included in Riaz (2009) but not in this work, does not display a silicate emission feature since it has no excess emission over the entire IRS range, so it most likely does not have a disk.

Among our sample, the weakest silicate features are found for 2MASS J04414489+2301513, IRAS 04125+2902, MHO 6, and DG Tau (see Figure 17). In the former two objects, the $10 \mu \mathrm{m}$ silicate feature is essentially absent. 2MASS J04414489+2301513 is a brown dwarf (spectral type of M8.5; Luhman 2006) that is likely a member of a quadruple system, with the closest companion (at 0.'105) a planetary-mass object (Todorov et al. 2010). Recent models suggest that it is surrounded by a very small disk with substantial grain growth $(\gtrsim 5 \mu \mathrm{m})$ and a moderate degree of dust depletion in the upper layers (Adame et al. 2011). IRAS $04125+2902$ is a transitional disk with a large inner hole (see Section 4.5), and, given the lack of a silicate emission feature, no small dust grains are present in the upper layers of its disk or the inner disk wall. MHO 6 has a weak silicate feature, but apparently a strong emission feature in the 13.5-14 $\mu \mathrm{m}$ region, which could be the signpost of molecules (the Q-branches of $\mathrm{C}_{2} \mathrm{H}_{2}$ and $\mathrm{HCN}$ are located at 13.7 and $14.0 \mu \mathrm{m}$, respectively; see also Pascucci et al. 2009). DG Tau is a high-accretion-rate object (e.g., Gullbring et al. 2000) with a spectral type of K6 (White \& Ghez 2001) that shows variations in its silicate feature, both in composition and strength, and even changing from emission into absorption (Wooden et al. 2000; Bary et al. 2009).

Based on the flux ratio at 11.3 and $9.8 \mu \mathrm{m}$, the most processed grains are found around UX Tau A, IRAS 04108+2910, DM Tau, 2MASS J04330945+2246487, 2MASS J04284263+2714039, 

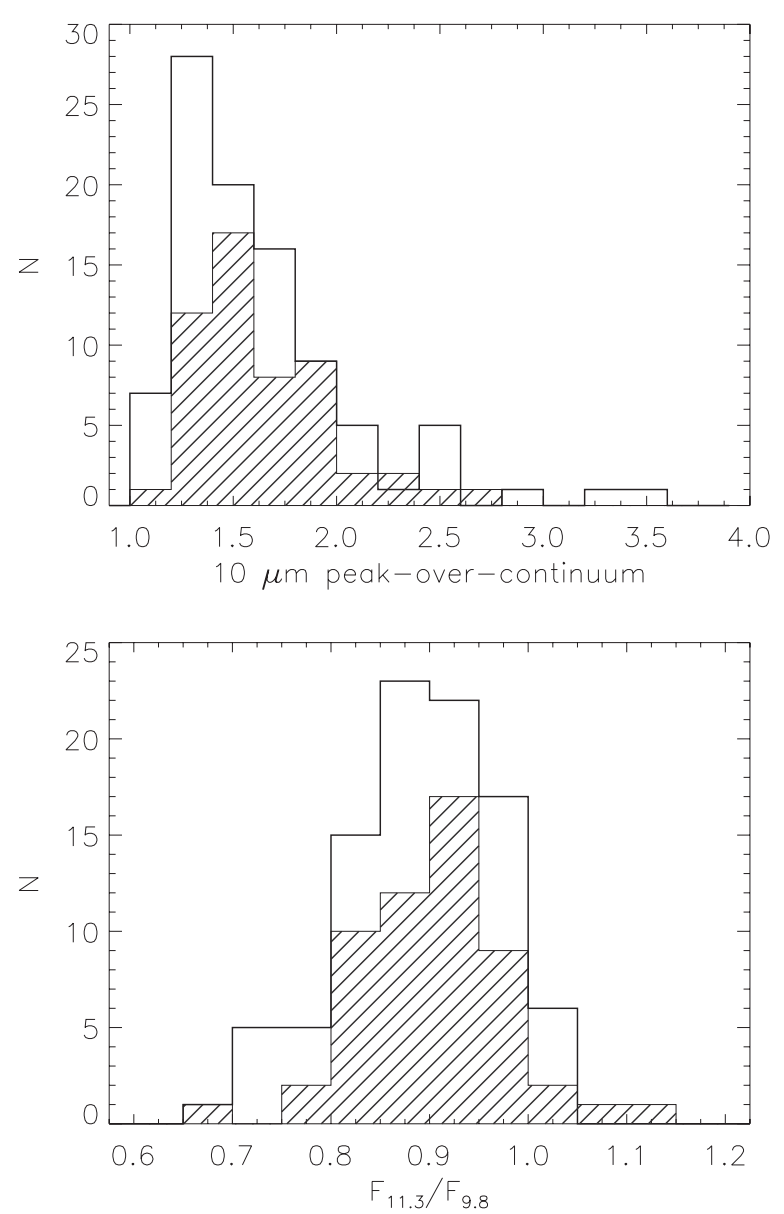

Figure 15. Histograms for the peak-over-continuum ratio of the $10 \mu \mathrm{m}$ silicate feature and for the 11.3 and $9.8 \mu \mathrm{m}$ flux ratio for the single T Tauri stars in our sample (white area) and for multiple systems (shaded area).

ZZ Tau IRS, DG Tau, 2MASS J04554535+3019389, and DH Tau (see Figures 18 and 19). However, in the case of UX Tau A the $F_{11.3} / F_{9.8}$ ratio cannot be used to assess grain processing, since this object displays a prominent PAH feature at $11.3 \mu \mathrm{m}$ (see also Furlan et al. 2006; Watson et al. 2009). Also, the silicate feature of 2MASS J04330945+2246487 is weak and of poor signal-to-noise ratio, so it is not clear whether it is dominated by processed grains. For the other objects, we estimated whether grain growth or crystallinity is mainly responsible for the large $F_{11.3} / F_{9.8}$ ratios by examining the shape of the silicate feature; the former objects are shown in Figure 18, while the latter ones are displayed in Figure 19.
DM Tau and ZZ Tau IRS have fairly symmetric, smooth, and broad silicate features (see Figure 18), which suggest the presence of large grains. Detailed modeling by Sargent et al. (2009) revealed that ZZ Tau IRS has a high mass fraction ( $95 \%)$ of large amorphous silicate grains in its inner disk. Similarly, DM Tau has a mass fraction of warm, large amorphous silicates of $\sim 75 \%$, and crystalline silicates contribute to about $30 \%$ of the mass of cooler dust. As noted by Sargent et al. (2009), DM Tau stands out among the transitional disks in Taurus, which typically are dominated by small amorphous silicate dust grains. We find that the $F_{11.3} / F_{9.8}$ ratios for $\mathrm{CoKu}$ Tau/4, GM Aur, IRAS $04125+2902$, and V410 X-ray 6 are more in line with the trend of decreasing ratios for increasing feature strength found for the rest of the sample (see Table 5).

IRAS 04108+2910, 2MASS J04284263+2714039, DG Tau, 2MASS J04554535+3019389, and DH Tau have silicate features that are more square in shape with several peaks between 9 and $12 \mu \mathrm{m}$, which are indicative of crystalline silicates (see Figure 19; the spectrum of DG Tau is shown in Figure 17 since it is also very weak). The silicate features of $2 \mathrm{MASS}$ J04284263+2714039 and DH Tau also stand out due to large $F_{11.3} / F_{9.8}$ ratios compared to objects with similar peak-overcontinuum ratios. Modeling of the silicate feature of DH Tau confirmed that the peaks at 9.3 and $\sim 11 \mu \mathrm{m}$ reveal enstatite in the disk surface layers where the mass fraction of crystalline grains amounts to about 45\% (Sargent et al. 2009).

More pristine grains, i.e., small, amorphous silicate grains as are found in the ISM, are present around LkCa 15, RY Tau, GM Aur, and IRAS 04370+2559, which have large peak-overcontinuum values for the $10 \mu \mathrm{m}$ feature $(\gtrsim 2.7)$ and small $F_{11.3} /$ $F_{9.8}$ ratios $(\lesssim 0.75)$. Indeed, their silicate features are smooth and peaked at $9.8 \mu \mathrm{m}$ (Figure 20).

All edge-on objects except for ZZ Tau IRS display a silicate absorption feature, in some cases with some emission, too, suggesting self-absorption by the disk. T Tau also exhibits a silicate absorption feature; while the northern component of this triple system is surrounded by a face-on disk which generates a silicate emission feature, the southern binary dominates the flux in the mid-infrared and is likely surrounded by an edge-on disk and an optically thin envelope, causing silicate absorption (see Ghez et al. 1991; Skemer et al. 2008; Ratzka et al. 2009).

\section{MEDIAN IRS SPECTRA}

Similar to Furlan et al. (2006) and Furlan et al. (2009b), we calculated the median IRS spectra for groups of T Tauri stars with similar characteristics in order to derive typical mid-infrared spectra for these groups. We normalized each
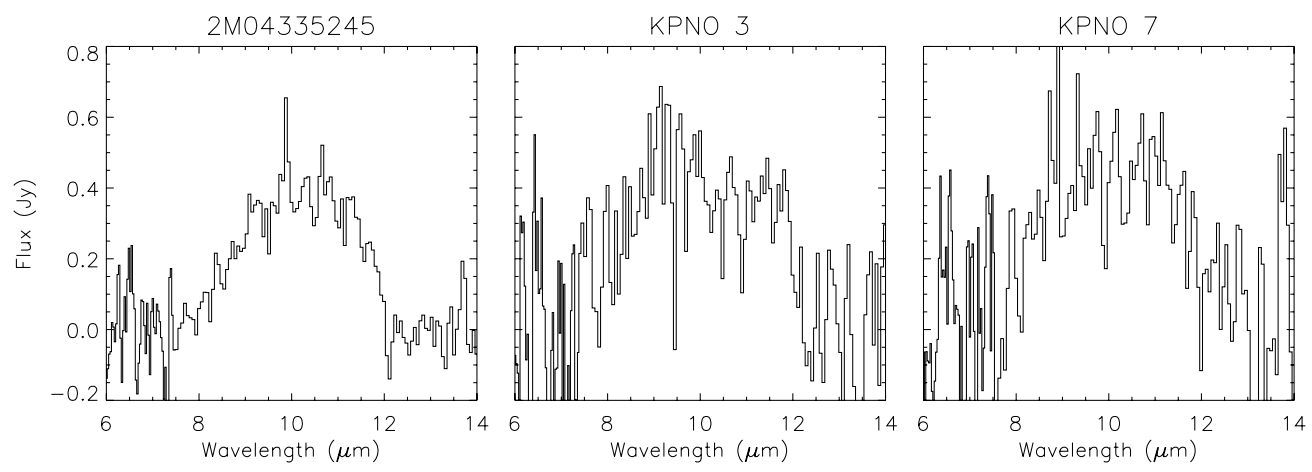

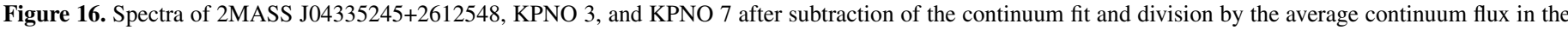

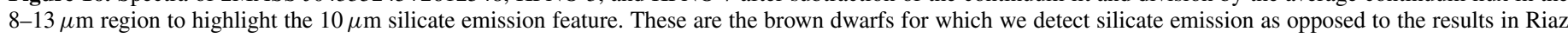
(2009). 

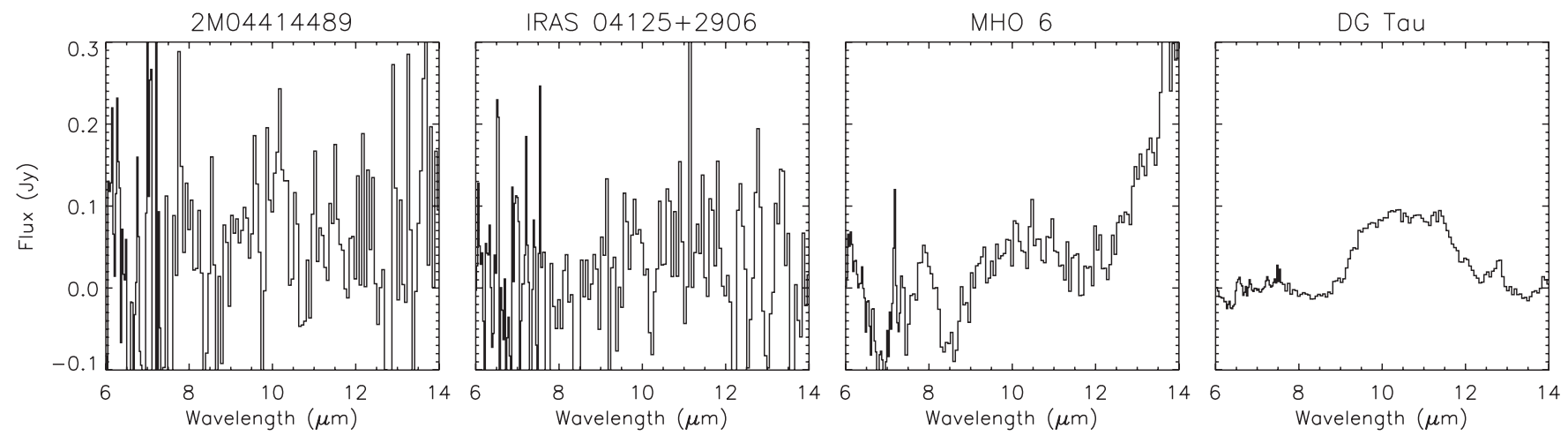

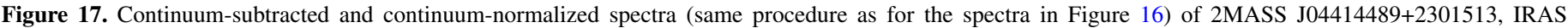
$04125+2902$, MHO 6, and DG Tau, which are the T Tauri stars with the weakest silicate emission features.
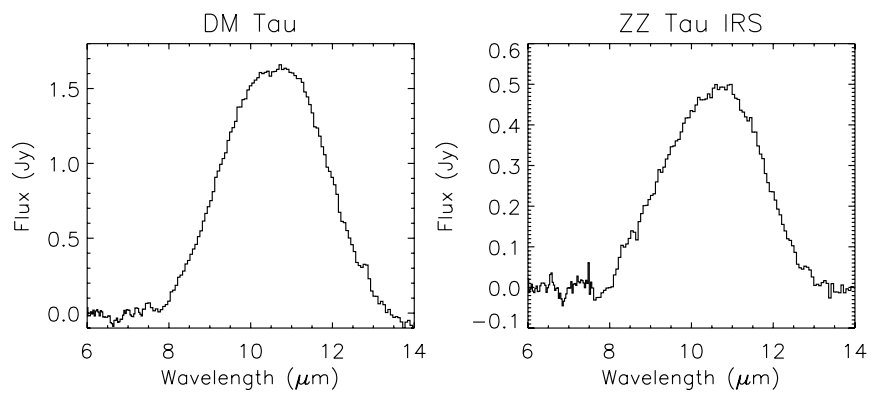

Figure 18. Continuum-subtracted and continuum-normalized spectra (same procedure as for the spectra in Figure 16) of DM Tau and ZZ Tau IRS which are the $\mathrm{T}$ Tauri stars most dominated by large grains as judged by the flux ratio at 11.3 and $9.8 \mu \mathrm{m}$ and the shape of their $10 \mu \mathrm{m}$ silicate features.

dereddened IRS spectrum to the median, dereddened $H$-band flux of the group (i.e., we multiplied each spectrum by the median $H$-band flux divided by the $H$-band flux of the object) before calculating the median flux at each wavelength. This procedure results in a median spectrum of the excess emission that does not strongly depend on stellar luminosities (D'Alessio et al. 1999).

For the medians, we chose three groups based on spectral types: one for objects with spectral types from K5 to M2 (73 spectra), one for M3-M5 spectral types (43 spectra), and one for M6-M9 spectral types (20 spectra). We only included objects that had a complete IRS spectrum from 5 to $36 \mu \mathrm{m}$; this led to exclusion of nine objects, which mostly affected the M6-M9 group, where a total of 24 objects were available based on spectral type alone. The K5-M2 group yields a spectrum of a typical young, low-mass star, while the M3-M5 and M6-M9 groups are representative of very low mass stars and mostly brown dwarfs, respectively.

Figure 21 shows the medians and the quartiles (defining the upper and lower limit where 50\% of the objects' fluxes lie) for the three groups of objects. Since fewer objects entered the M6-M9 median, it is more noisy than the other two medians. The medians were not scaled; therefore, the fact that the M3-M5 and M6-M9 medians are fainter than the K5-M2 median by factors of about 6 and 34, respectively, is an effect of lower stellar luminosities (which affect the normalization of the median) or lower disk emission for the later spectral types. The median $J$ - and $H$-band fluxes of the M3-M5 stars are both a factor of three fainter than those of the K5-M2 stars; these factors amount to 28 and 25 for the M6-M9 objects. Thus, assuming that the emission at these wavelengths is mostly photospheric, the difference between the three medians suggests that the excess emission from disks around later-type stars is also weaker. This can be understood in terms of decreased disk heating and smaller emitting area due to the lower stellar luminosities and smaller disk surface densities (which result in less flared disks that intercept less radiation from the star).

To estimate the emission from disks around stars of different spectral types, we assume a flared, optically thick disk heated by radiation from the star (see Ercolano et al. 2009). In this case, the emission from the disk relative to that from the star at each wavelength scales roughly as $\left(\lambda T_{*}\right)^{p}$, where $T_{*}$ is the temperature of the star (assumed to be a blackbody), and $p$ ranges from $5 / 3$ to 3 depending on the degree of flaring of the disk. With a stellar temperature ratio of about $1.2(\sim 4000 \mathrm{~K} / 3300 \mathrm{~K})$ and our measured photospheric emission ratio of $\sim 3$ for the K5-M2 and M3-M5 groups, we would expect the disk emission from the later-type objects to be a factor of 4-5 weaker than for
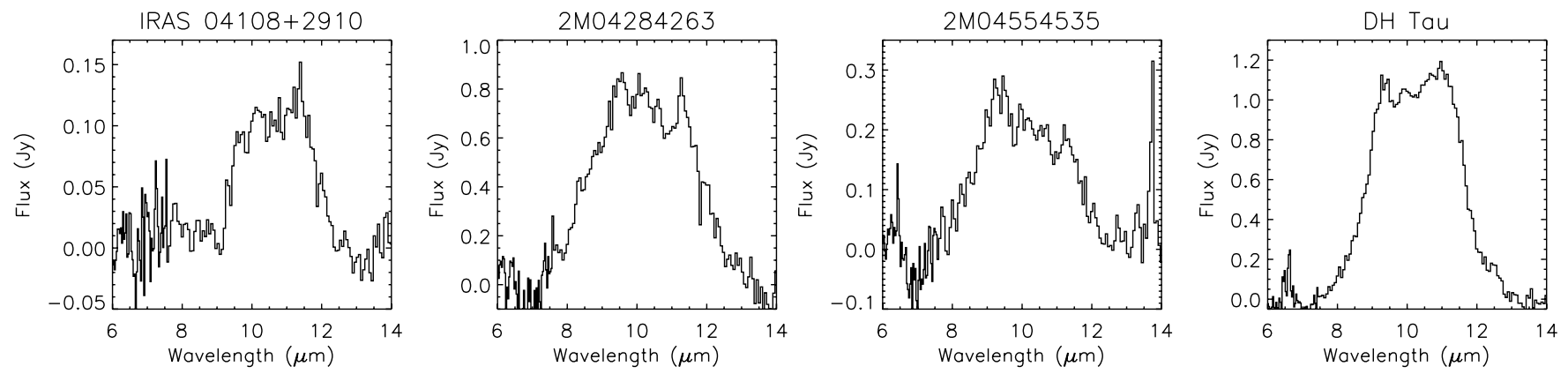

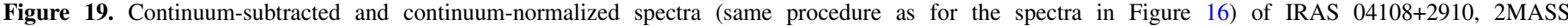

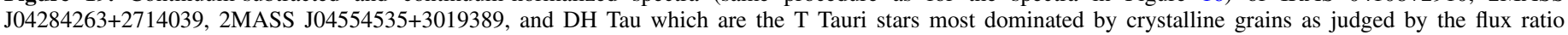
at 11.3 and $9.8 \mu \mathrm{m}$ and the shape of their $10 \mu \mathrm{m}$ silicate features. 

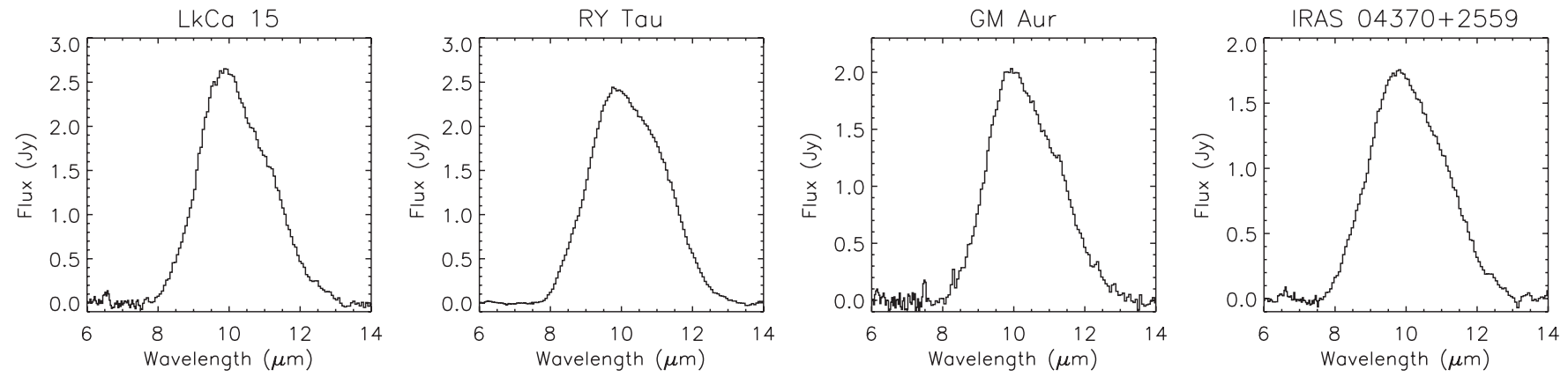

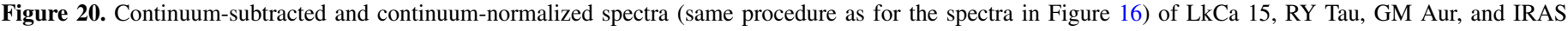
$04370+2559$, which are the T Tauri stars with the most pristine (i.e., sub- $\mu \mathrm{m}$, amorphous silicate) grains.

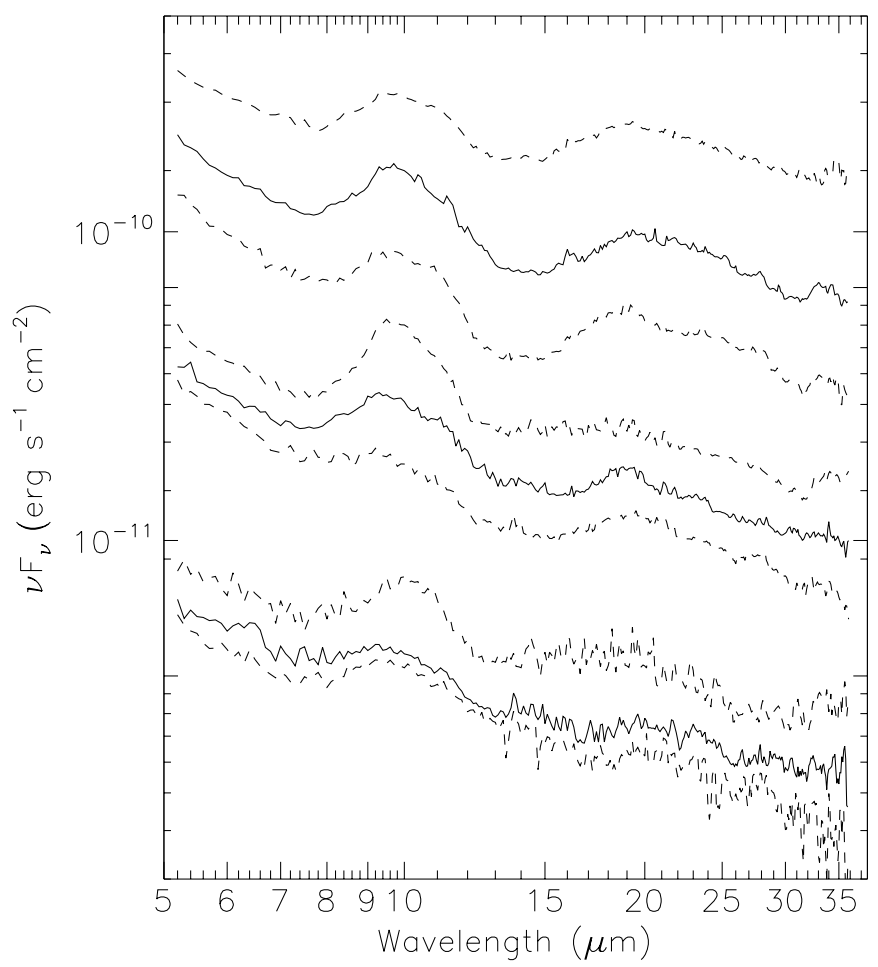

Figure 21. Median IRS spectra for T Tauri stars in Taurus (solid lines) and their quartiles (dashed lines). The upper set of spectra are the medians and quartiles for K5-M2 spectral types, the middle set represents M3-M5 spectral types, and the lower set M6-M9 spectral types. No scaling factor was applied to any of the medians or quartiles.

the K5-M2 objects, which is in rough agreement with what we observe for the two medians. A similar calculation for the K5-M2 and M6-M9 groups, for which the stellar temperature ratio of amounts to about $1.5(\sim 4000 \mathrm{~K} / 2700 \mathrm{~K})$ and the photospheric emission ratio is $\sim 26$, yields an estimated factor of 51-88 by which the disk emission from the M6-M9 stars is weaker than that from the K5-M2 stars. This factor is larger than what we observe, but our simple estimate gives the correct order of magnitude of the effect.

Overall, the shapes of the three medians are somewhat alike; both the M3-M5 and M6-M9 medians are slightly steeper over the entire IRS range $(5-36 \mu \mathrm{m})$, and the 10 and $20 \mu \mathrm{m}$ silicate emission features become less pronounced the later the spectral types included in the median. To compare the medians more quantitatively, we computed the 6-13 $\mu \mathrm{m}$ and $13-31 \mu \mathrm{m}$ spectral indices for the three medians and compared them to indices derived from accretion disk models (D'Alessio et al.
2006; Espaillat 2009; Adame 2010; L. Adame et al. 2011, in preparation); the results are shown in Figure 22. Some of the models are the same as the ones used in Section 5, but here we probe a larger parameter space: the models include three stellar masses $\left(0.5,0.2\right.$, and $\left.0.05 M_{\odot}\right)$ to represent $\mathrm{K} 7, \mathrm{M} 4$, and M7 spectral types, three mass accretion rates $\left(10^{-8}, 10^{-9}\right.$, and $10^{-10} M_{\odot} \mathrm{yr}^{-1}$ for the 0.2 and $0.5 M_{\odot}$ stars, and $10^{-10}, 10^{-11}$, and $10^{-12} M_{\odot} \mathrm{yr}^{-1}$ for the brown dwarfs), three inclination angles $\left(20^{\circ}, 40^{\circ}, 60^{\circ}\right)$, and four different settling parameters $\epsilon(0.001,0.01,0.1$, and 1.0$)$.

The 13-31 $\mu \mathrm{m}$ spectral indices for the M3-M5 and M6-M9 medians are lower than for the K5-M2 median, while the 6-13 $\mu \mathrm{m}$ spectral indices are slightly larger (i.e., less negative). Comparing these indices with those derived from models, we infer similar dust settling for the K5-M2 and M3-M5 groups, with $\epsilon$ between 0.001 and 0.01 , and somewhat lower mass accretion rates for the latter group (which is expected for very low mass stars; e.g., Muzerolle et al. 2003). Models of brown dwarf accretion disks calculated for typical mass accretion rates of substellar-mass objects $\left(\sim 10^{-11}-10^{-10} M_{\odot} \mathrm{yr}^{-1}\right.$; e.g., Muzerolle et al. 2005; Mohanty et al. 2005; Herczeg \& Hillenbrand 2008) suggest that values of $\epsilon$ between about 0.01 and 0.1 can explain the $n_{13-31}$ values for the M6-M9 members of Taurus (see also L. Adame et al. 2011, in preparation).

The increase in the 6-13 $\mu \mathrm{m}$ spectral index from the K5-M2 to the M6-M9 median could be understood in terms of the emission from the inner disk wall located at the dust sublimation radius, which dominates the flux in the $2-5 \mu \mathrm{m}$ region (Muzerolle et al. 2003; D'Alessio et al. 2005). For example, if the height of the inner disk wall decreases, the flux at $6 \mu \mathrm{m}$ will be reduced compared to that at $13 \mu \mathrm{m}$, where the disk emission is more prominent, resulting in a less steep 6-13 $\mu \mathrm{m}$ spectral index. The models of brown dwarf accretion disks seem to yield $n_{6-13}$ values that are somewhat lower than observed; this suggests that the inner disk component in these models needs to be adjusted (see L. Adame et al. 2011, in preparation).

In Figure 23, we compare the $n_{6-13}$ and $n_{13-31}$ indices for models with stellar masses of 0.2 and $0.5 M_{\odot}$. In general, both spectral indices are smaller for the $0.2 M_{\odot}$ models than for the $0.5 M_{\odot}$ ones. Thus, for two models with the same mass accretion rate, settling parameter, and inclination angle, but different stellar masses and luminosities, the model for the star with the lower mass and luminosity results in steeper continuum spectral indices. This trend would become even more pronounced if the models for the lower-mass stars used in the comparison also had lower mass accretion rates, as is observed (e.g., Muzerolle et al. 2003; Herczeg \& Hillenbrand 2008). The fact that we actually observe an increase in $n_{6-13}$ for the lower-mass objects suggests 


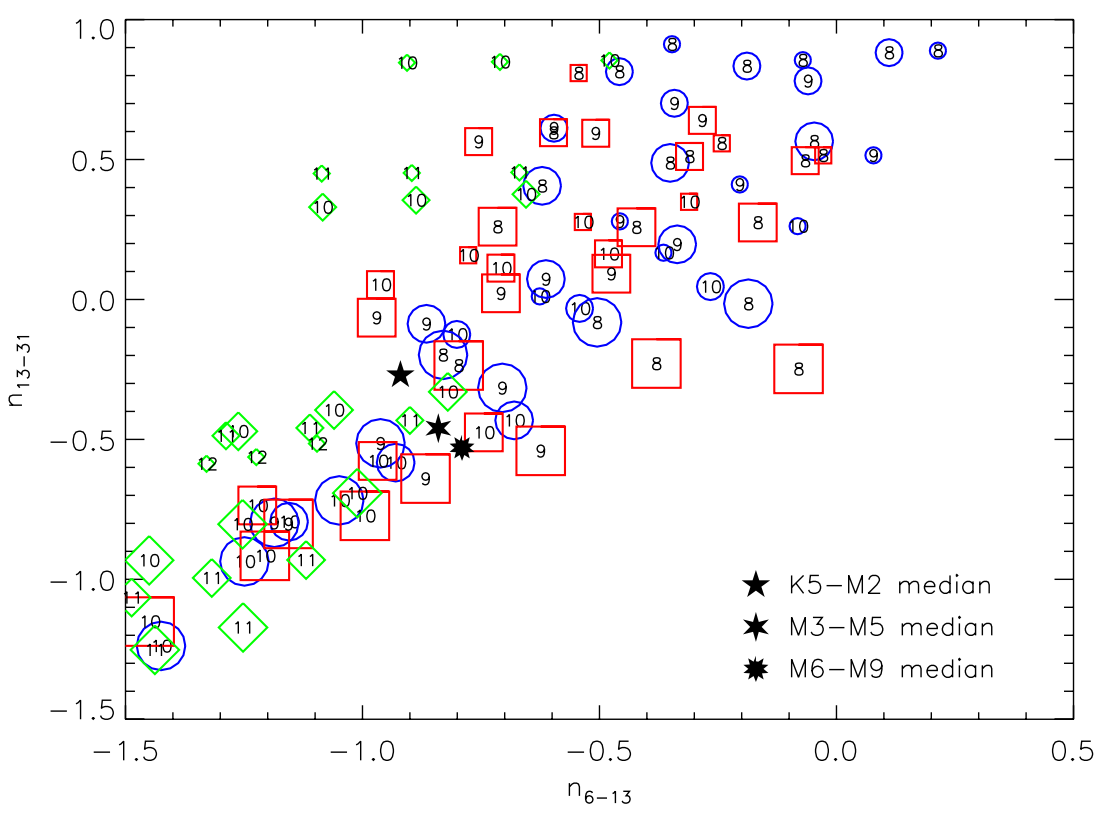

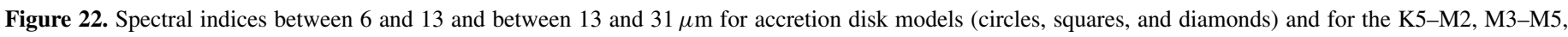

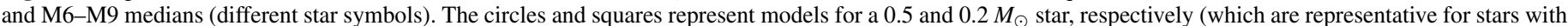

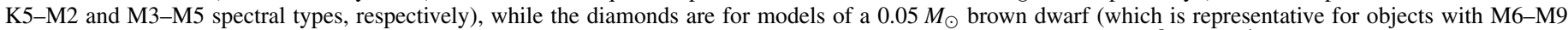

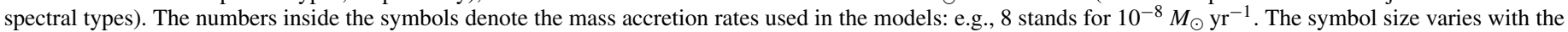
settling parameter $\epsilon$, with the smallest symbols for $\epsilon=1$ and the largest symbols for $\epsilon=0.001$.

(A color version of this figure is available in the online journal.)

that their models, which do not include variations in the inner disk wall, should incorporate reduced emission from this wall or the inner disk. On the other hand, the trend for the $n_{13-31}$ index, which is dominated by disk emission, agrees with the observational results. We conclude that the steeper $n_{13-31}$ values for the M3-M5 and M6-M9 medians are mostly a result of the lower stellar luminosity (and thus weaker stellar irradiation and disk emission) and also lower mass surface density of the disk, which depends on the mass accretion rate, stellar mass, and local disk midplane temperature as $\Sigma \propto \dot{M} M_{*}^{1 / 2} /\left(\alpha T_{c}\right)$ (see D'Alessio et al. 1998; $\alpha$ is a parameter that describes the turbulent viscosity and is set to 0.01 in the sets of models used here).

In Figure 24 we compare the K5-M2 median obtained in this work with the one from Furlan et al. (2009b). Our current sample of K5-M2 T Tauri stars is larger by 18 objects; 51 objects are included in both medians, four objects from the earlier median are excluded in the new one due to updated spectral types, and 22 objects are added to the new median for the same reason or because they were not part of the earlier sample. Of the 22 new objects, half are of K (K5-K7) and half of M (M0-M2) spectral type, so they are still representative of the full range of spectral types entering the K5-M2 median. The two medians shown in Figure 24 match quite closely, with the largest differences in the 5-8 $\mu \mathrm{m}$ region. The upper quartile from the current work seems to be flatter (i.e., a shallower slope over the mid-infrared spectral range), but roughly at the same flux level as the previously determined upper quartile, while the lower quartile is lower in flux than the one from the previous work (by about 20\%), but matches its shape almost precisely.

\section{DISCUSSION}

\subsection{Classification of Evolutionary State}

At an age of 1-2 Myr, most YSOs are still surrounded by circumstellar material in the form of a disk; a smaller fraction is still embedded in their natal envelopes. Extensive surveys of the Taurus star-forming region have shown about $65 \%$ of its pre-main-sequence population displays an infrared excess and is therefore accompanied by circumstellar material, with the fraction of excess objects declining for later spectral types (Luhman et al. 2010). Therefore, most pre-main-sequence stars in Taurus are either Class I or Class II objects. However, there is the possibility of a larger population of Class III objects, since likely not all of them are covered by surveys that target the denser regions of Taurus (Luhman et al. 2010).

Our classification using the "extinction-free" indices $n_{5-12}$ and $n_{12-20}$ (adopted from McClure et al. 2010) to identify the degree to which objects are embedded in circumstellar material (see Section 3.3) shows that mid-infrared data are crucial in determining disk- or envelope-dominated sources. To first order, the slope of the SED over the mid-infrared spectral range reveals whether a disk or an envelope is mostly responsible for the excess emission; the details of the IRS spectrum, such as the presence of features due to silicates or ices, can refine the classification. For example, there are a few Class I objects that appear to be disk-dominated based on their 5-12 $\mu \mathrm{m}$ spectral index, but ice and silicate absorption features, as well as models, suggest that an envelope is the main contributor to the thermal emission beyond about $15 \mu \mathrm{m}$ (see Furlan et al. 2008). Conversely, Class II objects that appear envelope-dominated, but have silicate emission features and flat or decreasing SEDs over the $\sim 3-30 \mu \mathrm{m}$ region are actually disk-dominated sources.

In some cases, additional information, such as high-resolution images, is necessary to distinguish the evolutionary stage of an object. In particular, the orientation of a system to line of sight can make it difficult to disentangle more evolved Class I objects and Class II objects. An example is Haro 6-5B, whose SED looks like that of a Class I object, but it is an edge-on disk, as confirmed by Hubble Space Telescope images (Krist et al. 1998). 

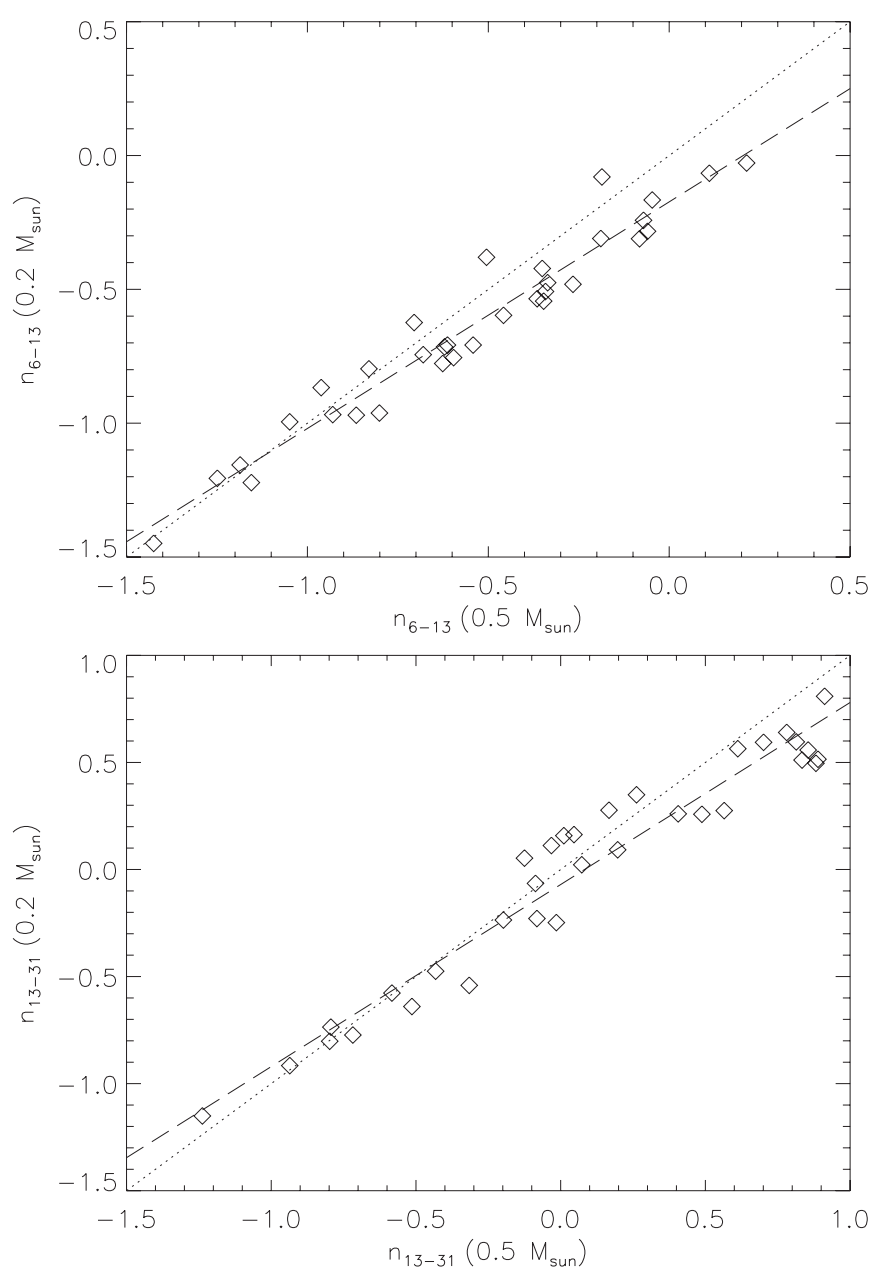

Figure 23. Comparison of the 6-13 $\mu \mathrm{m}$ and $13-31 \mu \mathrm{m}$ spectral indices for models with stellar masses of 0.2 and $0.5 M_{\odot}$. For each model pair, the mass accretion rate, settling parameter $\epsilon$, and inclination angle are the same. The dotted line represents a line with slope 1 and no offset, while the dashed line is a linear fit to the data.
Edge-on disks cannot be defined based on mid-infrared data alone; some of them display two peaks in emission, one at nearinfrared wavelengths and one in the mid- to far-infrared, as well as absorption features, but other objects are revealed just based on their unusually low luminosity or shape and width of their emission lines. Often high-resolution imaging and spectroscopy are needed to confirm the edge-on orientation of a disk. Among our sample of $\mathrm{T}$ Tauri stars, we singled out the more obvious cases of edge-on disks, but there are a few other objects that likely have high inclination angles (such as IC 2087 IR, V892 Tau, MHO 3). The boundary between an exactly edge-on disk and a highly inclined one is not well defined; the appearance of the SED will depend on other factors, such as the flaring angle of the disk (D'Alessio et al. 2006; Robitaille et al. 2006). In fact, the fraction of edge-on disks in our sample, which amounts to about $4 \%$ (or $12 \%$, if all 12 highly extinguished objects are actually oriented edge-on, too), suggests that disks in Taurus are on average quite settled, since models of well-mixed disks with only sub- $\mu \mathrm{m}$ dust grains would yield a fraction of edge-on disks of 30\% (D’Alessio et al. 1999).

Highly extinguished Class II objects can be difficult to classify before extinction correction; however, their 5-12 and 12-20 $\mu \mathrm{m}$ spectral indices reveal that their excess emission is dominated by a disk. In a few cases, part of the extinction could be due to a higher inclination angle along the line of sight causing the outer disk material to obscure the object. Fortunately, compared to other young star-forming regions, such as Ophiuchus (McClure et al. 2010), Serpens, and NGC 1333 (Winston et al. 2009), Taurus suffers from relatively little extinction, and therefore highly extinguished objects are rare.

\subsection{Disk Evolution in Low-mass Stars and Brown Dwarfs}

Using mid-infrared spectra, we can identify the evolutionary state of a disk: the spectral slope beyond $13 \mu \mathrm{m}$ is a measure for dust settling, and the $10 \mu \mathrm{m}$ feature reveals grain processing. There is a wide range in both parameters for the objects in our sample, and there is no strong trend with spectral type (and thus stellar mass). At first glance, this would suggest that

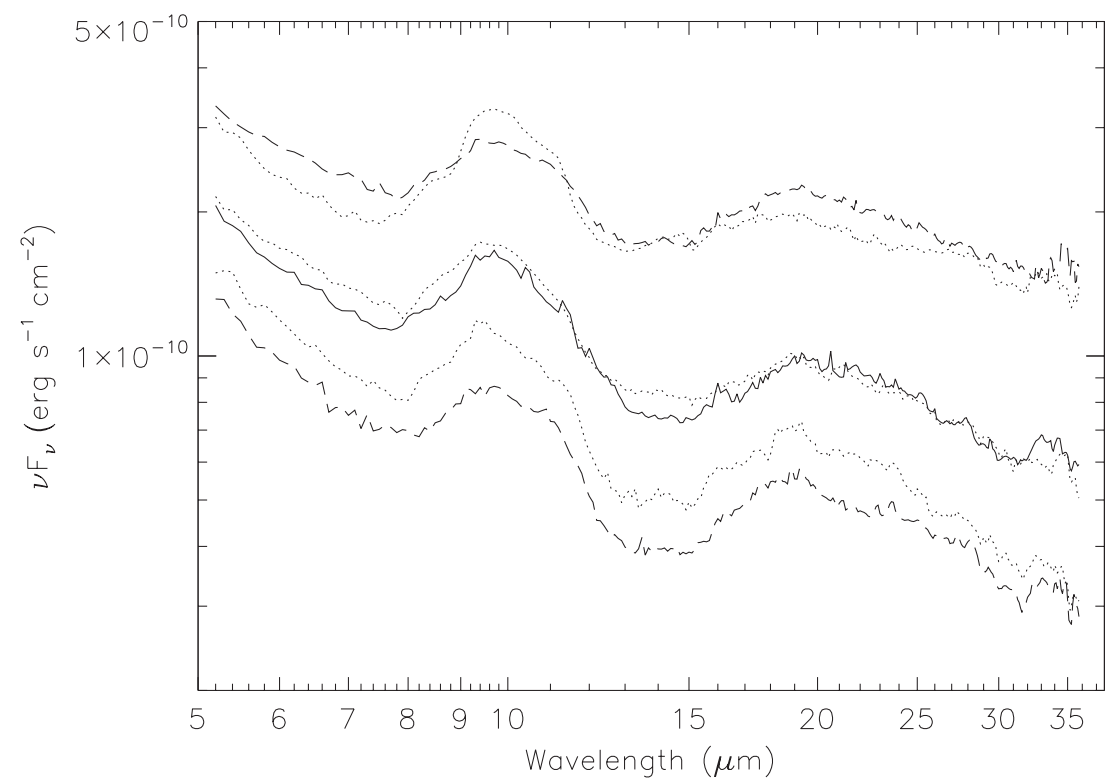

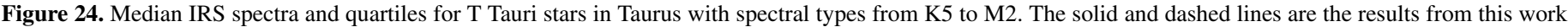

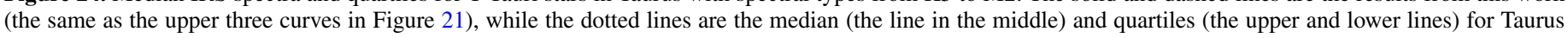
from Furlan et al. (2009b). 
disks around low-mass stars and brown dwarfs evolve on similar timescales.

Previous work suggested that disks around brown dwarfs could be more settled than those of low-mass stars, even though the SEDs of some brown dwarfs could be explained by a flared disk (Natta et al. 2002; Mohanty et al. 2004; Apai et al. 2005; Allers et al. 2006; Pascucci et al. 2009). A recent study by Szücs et al. (2010) involving the pre-main-sequence population in Chamaeleon I (Luhman et al. 2008) found that disks around very low mass objects (those with a spectral type of M4.75 and later) seem to be flatter than those around low-mass stars. Szücs et al. (2010) reached this conclusion by comparing the IRAC colors of the two samples and by applying model fits to the two median SEDs, which were constructed from 2MASS $J H K$, IRAC 3.6, 4.5, 5.8, and $8 \mu \mathrm{m}$, and MIPS $24 \mu \mathrm{m}$ photometry. The models required significant settling for disks around both the low-mass and very low mass stars, with reductions in disk scale heights about a factor of two larger in the latter group of objects.

Here we probe dust settling with the 13-31 $\mu \mathrm{m}$ SED slope, which is a better indicator of dust settling than IRAC colors (see D'Alessio et al. 2006). When looking at individual spectral type bins, we find that brown dwarfs do not have more steeply declining 13-31 $\mu \mathrm{m}$ slopes than low-mass stars; only when the median emission from disks around low-mass stars (K5-M2 spectral types) and very low mass stars and brown dwarfs (M3-M9 spectral types) is compared, the resulting $n_{13-31}$ values are lower for the latter groups of objects.

A comparison of the median 13-31 $\mu \mathrm{m}$ slopes with accretion disk models (D'Alessio et al. 2006; Espaillat 2009; Adame 2010) suggests that the lowest-mass stars have, on average, dust depletion in their upper disk layers that is very similar to that of disks around low-mass stars. The same conclusion applies to brown dwarf disks. Thus, we do not find a higher degree of dust settling in disks around very low mass objects. The steeper 13-31 $\mu \mathrm{m}$ spectral index in M3-M9 objects as compared with $\mathrm{K} 5-\mathrm{M} 2$ ones is due in part to smaller stellar luminosities and thus weaker disk emission for stars with late spectral types. The weaker disk emission is caused by both lower photospheric fluxes and stellar temperatures (Ercolano et al. 2009), with the latter dependence equivalent to a dependence on radius and equilibrium temperature in the disk. For cooler stars, a given temperature in the disk is reached at smaller radii, resulting in a smaller emitting area and thus disk emission. If accretion is factored in, lower mass accretion rates and stellar masses result in lower surface densities of the disk (D'Alessio et al. 1998, 1999; Adame 2010), causing decreased flaring and thus a smaller irradiation surface to intercept the stellar radiation. As expected, we find that our M3-M5 and M6-M9 medians are about a factor of 6 and 34, respectively, lower than the K5-M2 median.

We note that even though stellar photospheres become redder for later spectral types ( M4; see Luhman et al. 2008, 2010), they do not have a very significant effect on the measured 13-31 $\mu \mathrm{m}$ SED slope. Compared to a K7 star, a brown dwarf photosphere has a [8] - [24] color that is about $0.2 \mathrm{mag}$ larger (Luhman et al. 2010), which results in a difference in the photospheric SED slope of $\sim 0.15$. Therefore, midinfrared spectral indices of brown dwarf disks appear less steep. To remove the effect of different photospheric colors, the $n_{13-31}$ values of brown dwarfs would have to be corrected by $\sim-0.15$. This change is relatively minor and does not affect our conclusion that disks around very low mass objects (M3-M9) have similar degrees of dust settling as disks around low-mass stars (K5-M2).

The $10 \mu \mathrm{m}$ silicate emission feature appears weaker for latetype objects, and the dust seems to be more dominated by processed grains. This also holds true when we compare our results to those derived from a large sample of Spitzer IRS spectra of Herbig Ae/Be stars (Juhász et al. 2010). Disks around Herbig Ae/Be stars tend to have stronger silicate features and a somewhat lower degree of dust processing than brown dwarf disks. Their silicate features are also, on average, stronger than those of $\mathrm{T}$ Tauri disks, but they appear to have similarly processed grains. These results agree with previous findings (Apai et al. 2005; Pascucci et al. 2009; Riaz 2009) and could be a selection effect, since in brown dwarfs the disk regions contributing most to the emission at $10 \mu \mathrm{m}$ lie at smaller radii than in low-mass stars (Kessler-Silacci et al. 2007). It is in these inner regions that grain growth happens fastest (e.g., Brauer et al. 2008), and crystallization of amorphous grains can occur close to the star, where temperatures are high enough for annealing of the grains (e.g., Fabian et al. 2000). In addition, the degree of flaring of the disk, and shocks, turbulence and mixing within it, will have an effect on the types of grains that are detected in the optically thin surface layers of the disk (Harker \& Desch 2002; Gail 2004; Birnstiel et al. 2011).

A large $13-31 \mu \mathrm{m}$ spectral index and an unusually strong $10 \mu \mathrm{m}$ emission feature can reveal disks with inner holes or gaps, respectively. Interestingly, only one of the objects with a spectral type later than M6 potentially has a gap in its disk. However, as mentioned above, in late-type stars the region probed by mid-infrared wavelengths is located closer in to the star; moreover, the disk emission relative to that of the star at any given wavelength is smaller for lower-mass stars, making it difficult to detect small (AU-scale) inner disk holes in the mid-infrared (Ercolano et al. 2009).

Of the transitional disks in our Taurus sample, all but one have spectral types in the K7-M1 range. This agrees with the findings of Muzerolle et al. (2010), who determined that the transitional disks in regions that are up to $\sim 3$ Myr old all have spectral types earlier than M2. This could point to different clearing mechanisms in disks depending on stellar mass.

As found in Furlan et al. (2009b), we confirm that about $20 \%$ of Class II objects in Taurus display an equivalent width of the $10 \mu \mathrm{m}$ silicate feature that cannot be explained by continuous accretion disk models. If the strong silicate feature is associated with disk gaps, it could imply that this stage lasts for a few $10^{5}$ years, or, if cyclical, a gap would survive for less than this time period (see also Watson et al. 2009; Furlan et al. 2009b). This latter scenario would match gap openings by forming planets, which will migrate on the viscous timescale of the disk (Ward 1997; Zhu et al. 2011). The presence of planets in these disks is also suggested by the variability detected in the mid-infrared spectra of pre-transitional disks by Espaillat et al. (2011). In that work, the authors suggest that disk warps induced by planets can change the height of the inner disk wall, which affects the mid-infrared emission, and also lead to shocks and collisions in the disk, which could change the composition and amount of the dust. Recent high-contrast imaging of the pre-transitional disk LkCa 15 revealed a pericenter offset of the disk gap, which also points to the dynamical effects of one or more planetary companions (Thalmann et al. 2010). Further monitoring of pre-transitional disks is necessary to confirm this hypothesis. 
The small fraction of objects with large $n_{13-31}$ values would suggest a transitional disk stage of several $10^{4}$ years. However, we caution that the fraction of transitional disks also depends on how such disks are defined; some authors also include pretransitional disks (those with gaps in their optically thick disk) or "evolved" disks (those with an overall low infrared excess) in their sample of transitional disks, which increases their fraction and thus the timescale for the transitional stage (Lada et al. 2006; Sicilia-Aguilar et al. 2008; Currie et al. 2009). Also, circumbinary disks should be excluded from the transitional disk sample, since they are not in a transition stage (see the example of CoKu Tau/4; Ireland \& Kraus 2008; Nagel et al. 2010), but high-resolution observations are not always available to reliably identify true transitional disks.

In the Taurus sample presented in this work, we have two confirmed transitional disks (DM Tau, GM Aur) and three likely ones (V410 X-ray 6, IRAS 04125+2902, and V410 $\mathrm{X}$-ray 2), four confirmed pre-transitional disks (LkCa 15, UX Tau A, RY Tau, and IP Tau), but about two dozen more awaiting confirmation (among them MHO 3, IRAS 04370+2559, GK Tau, JH 112 A, 2MASS J04202606+2804089, and 2MASS J04390525+2337450), and four "evolved" disks, of which one has a steep SED slope suggesting a very settled or optically thin disk (2MASS J04330945+2246487), and three likely have optically thin disks with inner holes (V819 Tau, RX J0432.8+1735, and $\mathrm{LkCa}$ 19).

\subsection{Multiplicity}

As can be seen from Table 3, a large number of objects in our sample consists of multiple components; in fact, about one-third of the $\mathrm{T}$ Tauri stars in this work have known companions. The separation between components varies from a few hundredths of an arcsecond to tens of arcseconds, which, at a distance of $140 \mathrm{pc}$ to Taurus (Bertout et al. 1999), corresponds to a few AU to several thousand $\mathrm{AU}$, respectively. We did not consider companions at distances larger than about $20^{\prime \prime}$ for reasons given in Section 3.2.

The fraction of multiple systems in our sample is lower than that derived from surveys for companions in the 3-5000 AU range in Taurus, which amounts to $\sim 66 \%-75 \%$ (Kraus et al. 2011). Since not all objects in our sample have been targeted by multiplicity surveys (in particular the very low mass stars and brown dwarfs), it is likely that more systems actually consist of multiple components.

As noted in Furlan et al. (2006), the IRS spectra often contain the combined emission of a multiple system, especially if components are within a few arcseconds of each other; the narrowest IRS slit is 3".6 wide (SL), which is roughly the same as the average separation between companions in our sample. However, the infrared flux ratios listed in Table 3 suggest that for almost two-thirds of systems either one component (i.e., one circumstellar or circumbinary disk) clearly dominates the midinfrared emission (flux ratio of $\sim 4$ or larger), or two disks in a binary system contribute about equally (flux ratio $\sim 1-1.2$ ).

It is interesting how varied the higher-order multiple systems are; in some cases all components are surrounded by accretion disks, while in other cases the circumstellar material around the various components is in different evolutionary stages (see, e.g., White et al. 1999; White \& Ghez 2001; Hartigan \& Kenyon 2003; Duchêne et al. 2003; McCabe et al. 2006). This suggests that disk evolution is strongly influenced by initial conditions and that the timescales of disk dissipation vary accordingly. Among binaries, mixed systems (i.e., consisting of a CTTS and WTTS) are the exception rather than the rule, indicating that in general disks in a binary system evolve on similar timescales, possibly regulated by replenishment of material from a circumbinary disk (see also Prato \& Simon 1997).

In some cases, a binary companion can truncate the outer circumprimary disk; this truncation will be detected in the midinfrared if the companion lies at a distance of at most a few AU and thus can truncate the disk at just several tenths of an AU (Artymowicz \& Lubow 1994). This truncation will result in an SED slope that is steeper than $-4 / 3$; a well-studied example is the disk around SR20 in Ophiuchus (McClure et al. 2008). We found four objects in Taurus with such steep SED slopes; none of them has known companions, but since they are very low mass stars (M4.5-M6), they could have undetected substellarmass companions. Recent models of the circumprimary disk of the brown dwarf 2MASS J04414489+2301513, which has a 15 AU planetary-mass companion (Todorov et al. 2010), suggest an outer disk radius of only $0.2-0.3 \mathrm{AU}$ and thus likely truncation by the companion (Adame et al. 2011).

We found that settling, dust growth and processing, and the formation of radial structure in the disk are not affected by the multiplicity of a system. This result matches the analysis of Pascucci et al. (2008) using a subset of the Taurus sample and also that of Manoj et al. (2011) involving Class II objects in the slightly older Chamaeleon I region. However, we note that close binaries, which are often not resolved in multiplicity surveys, can have an effect on disk evolution (e.g., Bouwman et al. 2006).

In a study of disks in the Ophiuchus star-forming region, McClure et al. (2010) found that the equivalent width of the $10 \mu \mathrm{m}$ silicate feature decreased for binaries with smaller separations, especially for $\lesssim 120$ AU. They interpreted this as circumprimary disks that are truncated at smaller outer radii for closer binary components; objects with small circumprimary disks would not have sufficient mass to form planets that can clear gaps in their disks, and therefore they would not display unusually large $10 \mu \mathrm{m}$ equivalent widths. When examining the dependency of $\mathrm{EW}(10 \mu \mathrm{m})$ values on the binary separation for the T Tauri stars in Taurus (for higher-order multiples, we used the separation between primary and secondary components), we find no clear trend with separation. For the multiple systems among the $\mathrm{EW}(10 \mu \mathrm{m})$ outliers identified in Figure 8, two-thirds have separations larger than $100 \mathrm{AU}$, while the remaining onethird has separations less than 50 AU. Even though circumprimary disks in the latter group of objects would be truncated at $\lesssim 20 \mathrm{AU}$, circumbinary disks likely play a role by either being the main disk component (for the closest binaries) or resupplying the primary disks. They could thus provide more material for planet formation, and these $\operatorname{EW}(10 \mu \mathrm{m})$ outliers could still be consistent with the presence of gap-opening planets in their disks.

\section{CONCLUSIONS}

1. We have presented all IRS spectra of $\mathrm{T}$ Tauri stars with infrared excesses (Class II objects) in Taurus observed during the Spitzer cold mission, as well as a few newly observed Class 0 and Class I objects. This sample contains a large majority of the known Class II objects in Taurus, and comprises the largest database of mid-infrared spectra for any single star-forming region. It is also the largest sample of mid-infrared spectra for disks of low-mass stars and brown dwarfs.

2. The extinction-free indices $\left(n_{5-12}, n_{12-20}\right)$ from McClure et al. (2010) are a useful means to distinguish between 
envelope- and disk-dominated sources, even in a region like Taurus where most $\mathrm{T}$ Tauri stars suffer from little extinction along the line of sight. The majority of objects in Taurus are disk-dominated; among the sample of objects with IRS spectra, there are about 30 known Class 0/I objects whose SEDs are rising in the mid-infrared and typically display strong ice and silicate absorption features. We confirm one new Class I member of Taurus, SST Tau 041831.2+282617, and determine that the mid-infrared emission of IRAS $04301+2608$ is actually dominated by a tenuous envelope likely seen at a high inclination angle.

3. Only $12 \mathrm{~T}$ Tauri stars in our sample are extinguished by more than $A_{J}=2.5$; after applying extinction corrections to their IRS spectra, they appear as typical Class II objects with 10 and $20 \mu \mathrm{m}$ silicate emission features. The most highly extinguished object in our sample, [GKH94] 41, was suggested to be an embedded proto brown dwarf, but our dereddened IRS spectrum identifies it as disk-dominated, with possibly even a gap in its disk.

4. The IRS spectra and SEDs of edge-on disks do not always display distinct features that would set them apart from less inclined T Tauri disks. This could be a reflection of different disk flaring or just slight differences in the inclination angle. However, most edge-on disks exhibit some ice absorption features, and in several cases their SEDs show a peak at near-infrared wavelengths and a rising slope beyond about $10 \mu \mathrm{m}$.

5. The mid-infrared spectra of $\mathrm{T}$ Tauri stars in Taurus uncover differences in disk structure and dust properties among the objects in our sample. As shown in previous work, the $13-31 \mu \mathrm{m}$ spectral index is a good indicator for the degree of dust settling in a disk, and the strength (peakover-continuum ratio) and shape (11.3 and $9.8 \mu \mathrm{m}$ flux ratio) of the $10 \mu \mathrm{m}$ silicate emission feature reveal whether dust grains are pristine (i.e., ISM-like) or have grown or become more crystalline. We find that most disks have experienced substantial dust settling, and the dust shows signs of processing (growth and crystallization).

6. Very low mass stars and brown dwarfs have in general weaker excess emission at wavelengths shortward of $5 \mu \mathrm{m}$ than stars at higher masses. Also, their $10 \mu \mathrm{m}$ silicate emission features suggest a higher degree of dust processing than in their higher-mass counterparts. Agreeing with previous findings, more processed dust (larger and/or more crystalline grains) is accompanied by weaker silicate features. However, after comparing the $n_{6-13}$ and $n_{13-31}$ spectral indices of objects in the K5-M2, M3-M5, and M6-M9 spectral type ranges with indices derived from accretion disk models, we find that very low mass objects do not have a higher degree of dust settling than low-mass stars.

7. The median IRS spectra for T Tauri stars with K5-M2, M3-M5, and M6-M9 spectral types are somewhat similar in shape, but the latter two medians are lower in flux. This can be explained by decreased disk emission due to lower disk surface density and impinging irradiation flux (caused by the smaller stellar luminosities) in latertype stars. The stellar temperature sets the radius at which a certain equilibrium temperature is reached in the disk, resulting in smaller radii (and a smaller emitting area) for cooler stars. Thus, in very low mass stars and brown dwarfs the regions probed in the mid-infrared lie closer to the star than in disks around higher-mass stars. This could explain why the dust in these objects appears more processed.

8. With the availability of IRS data, we were able to confirm three additional transitional disks in Taurus that were previously identified with photometry (V410 X-ray 6, IRAS $04125+2902$, and V410 X-ray 2), bringing the total to five transitional disks. These disks are characterized by inner holes and therefore lack excess emission at shorter wavelengths, but display a steep rise in the SED beyond about $12 \mu \mathrm{m}$. Among our sample, even fewer objects (V819 Tau, RX J0432.8+1735, and LkCa 19) have low infrared excess emission and some inner disk clearing, suggesting the presence of an optically thin disk with an inner hole; in two of these objects, the IRS data reveal the details of the infrared excess for the first time. These disks could be at an advanced transitional stage or already debris disks consisting of second-generation dust.

9. While transitional disks can be identified by their large $n_{13-31}$ spectral index, the equivalent width of the $10 \mu \mathrm{m}$ silicate emission feature can be used to identify objects with unusually strong optically thin dust emission, which could arise from dust in disk gaps. These objects have been termed pre-transitional disks, and there is mounting evidence that they are indeed characterized by gaps, not holes, in their optically thick disks. Their fraction of $\sim 20 \%$ in our Taurus sample suggests a longer pre-transitional than transitional timescale, or the stage in which a gap is present is shorter, but would occur more than once during a disk's lifetime. This could point to forming planets in the disk, which would open up a gap and then migrate inward.

10. Disk evolution seems to proceed on similar timescales in low-mass stars and very low mass objects, including brown dwarfs, since they all display signs of dust settling and processing. In the latter group of objects, different disk clearing mechanisms might apply, given that we identified only one object with a spectral type later than M6 and potentially a gap in its disk, but it is important to keep in mind that the mid-infrared probes different radial locations in disks around stars with different luminosities.

11. One-third of the objects in our sample are known to belong to multiple systems. We found no difference in the distribution of $n_{13-31}$, EW(10 $\left.\mu \mathrm{m}\right)$, peak-over-continuum, and $F_{11.3} / F_{9.8}$ ratios for single and multiple systems. Therefore, as far as the population of T Tauri stars in Taurus is concerned, multiplicity has no effect on the evolution of a disk in terms of dust processing, settling, and gap/hole formation. For individual objects, a companion located at a certain distance can cause an inner disk hole (e.g., CoKu Tau/4; Ireland \& Kraus 2008) or a truncated outer disk (e.g., objects with $n_{13-31}$ below $-4 / 3$ ).

Our large sample of IRS spectra of YSOs in Taurus is an important addition to the mounting data sets available for this star-forming region, both from ground- and spacebased observatories and extending over a wide range of the electromagnetic spectrum. While photometry can provide SEDs and thus an idea of the distribution of circumstellar material, only IRS spectra reveal the details of the mid-infrared emission, both from the optically thick continuum and the optically thin dust. Since objects in Taurus cover all evolutionary stages, this data set will be a benchmark for studies of other, young starforming regions and thus plays a vital role in advancing our understanding of star and planet formation. 
This work is based on observations made with the Spitzer Space Telescope, which is operated by the Jet Propulsion Laboratory (JPL), California Institute of Technology (Caltech), under a contract with NASA. Support for this work was provided by NASA through an award issued by JPL/Caltech. E.F. was supported by NASA through the Spitzer Space Telescope Fellowship Program, through a contract issued by JPL/Caltech under a contract with NASA. K.L. was supported by grant AST-0544588 from the National Science Foundation. The Center for Exoplanets and Habitable Worlds is supported by the Pennsylvania State University, the Eberly College of Science, and the Pennsylvania Space Grant Consortium. C.E. was supported by the National Science Foundation under Award No. 0901947. This publication makes use of data products from the Two Micron All Sky Survey, which is a joint project of the University of Massachusetts and the Infrared Processing and Analysis Center/Caltech, funded by NASA and the NSF. It has also made use of the SIMBAD and VizieR databases, operated at CDS (Strasbourg, France), NASA's Astrophysics Data System Abstract Service, and of the NASA/IPAC Infrared Science Archive operated by JPL, Caltech, under contract with NASA.

Facility: Spitzer(IRS)

\section{APPENDIX}

Here we list objects observed in a non-standard mode or which had data reduction problems.

2MASS J04141188+2811535. MHO 2 and CIDA 1 saturated the lower part of the blue and red peak-up array, respectively. This caused some additional, low-level noise in the 5.2-5.4 and $7.05-7.45 \mu \mathrm{m}$ regions of the spectrum. V773 Tau entered both the LL2 and LL1 slits. While the reduction of the LL2 spectrum presented no problems, in LL1 a double peak was present and required a user-defined extraction at the requested nod position.

2MASS J04202144+2813491. This very faint object had to be extracted with optimal extraction. It also required sky subtraction in LL2 and LL1 using line fits to the background.

2 MASS J04210795+2702204. This object was only observed with SH. In addition, no sky observation was obtained, but since it is fairly bright, the background contamination should be negligible $(\lesssim 5 \%)$.

2MASS J04390163+2336029 and IRAS 04191+1523. These objects were observed with SL, SH, and LL1, so there is a small gap in wavelength coverage between 19.4 and $20.8 \mu \mathrm{m}$.

2MASS J04390525+2337450. Only SH and LH were observed for this object.

2MASS J04390396+2544264. In LL2, the spectrum had to be extracted using optimal extraction to minimize the impact of a cluster of bad pixels in nod 2 in the 19.1-19.7 $\mu \mathrm{m}$ region.

2MASS J04400067+2358211. In nod 1 of LL2, a bright source appeared at the opposite nod position and thus required a user-defined extraction at the requested nod position.

2MASS J04414489+2301513. This object was extracted using optimal extraction, since it is a close double source in LL2 and LL1 (the other source entering the LL slit is the binary 2MASS J04414565+2301580).

2MASS J04414825+2534304. Only SL was observed for this object.

2MASS J04554801+3028050. In LL, this object is blended with 2MASS J04554757+3028077, which is weakly or not accreting any more (Herczeg \& Hillenbrand 2008) and therefore unlikely to contribute significantly to the combined flux. LL had to be scaled by a factor of 0.8 to match the flux level of SL at $14 \mu \mathrm{m}$.

CFHT 4. This object was only observed with SL1 and LL1, so there is no spectrum available in the 5-7.7 $\mu \mathrm{m}$ and $14-20.6 \mu \mathrm{m}$ regions. The first cycle of the first nod of SL1 was corrupted and therefore not used in the reduction.

CIDA 1. CW Tau and V773 Tau saturated the red peakup array and caused parts of the array where SL1 lies to be more noisy. However, this affected the SL1 spectrum of CIDA 1 between 8.9 and $10.2 \mu \mathrm{m}$ only at the level of a few percent.

CW Tau. The SL2 spectrum from 7.03 to $7.57 \mu \mathrm{m}$ had to be masked since MHO 3 saturated the blue peak-up array and caused striping and noise in the lower part of the array.

FV Tau/c. Only the SL spectrum could be extracted, since LL is mostly contaminated by FV Tau, which lies at a distance of $12^{\prime \prime}$. Of the two binary components in the FV Tau/c system, the secondary likely dominates the infrared emission.

FY Tau. The LL spectrum had to be extracted using optimal extraction, since FZ Tau entered the LL slit and created a double source in LL2 and LL1.

Haro 6-5B. FS Tau entered the LL slit, causing a double peak in this module. Both SL and LL were extracted using optimal extraction. Likely due to some mispointing, there are fringes in LL1.

Haro 6-28. LL1 had to be extracted at the requested nod position, since HP Tau also entered the LL slit (but is well separated spatially from Haro 6-28).

HH 30. In Furlan et al. (2008) we were only able to extract the SL1 and LL spectrum of this object. Using better sky subtraction in SL2, for this work we extracted also the SL2 spectrum.

IRAM 04191+1522. Only LL was observed for this object, which is very faint in LL2. Vertical stripes affected the array in nod 2; we only used nod 1 of LL2 for the final spectrum.

IRAS 04125+2902. The second cycle of the first nod of SL2 was not usable, so it was not included in the reduction.

IRAS $04301+2608$. The SL observations were somewhat mispointed; the extracted SL spectrum had to be multiplied by 1.4 to match the flux level of LL at $14 \mu \mathrm{m}$.

JH 112. The two components of this binary system are separated by only 6.'6. In campaign 4 we observed JH 112 A in mapping mode (see Furlan et al. 2006) and JH 112 B in staring mode. The LL spectrum of both JH 112 A and B is the combined spectrum of the system, since the LL slit in 10.'6 wide. However, JH 112 A is brighter than the B component by a factor of 2-3 in the four IRAC bands (Luhman et al. 2010), so it is reasonable to assume that it dominates the IRS spectrum. In fact, the LL spectrum of JH $112 \mathrm{~A}$ had to be scaled by a factor of 0.85 to match the flux level of SL at $14 \mu \mathrm{m}$. Here we present for the first time the SL spectrum of JH $112 \mathrm{~B}$.

KPNO 6, KPNO 12. Only SL1 was observed for these objects.

$K P N O$ 7. DK Tau saturated the blue peak-up array during the SL1 observation, but it only affected the SL1 spectrum beyond $14.1 \mu \mathrm{m}$ (which is trimmed anyway due to poorer calibration beyond $14 \mu \mathrm{m}$ ). In LL1, the spectrum required a user-defined extraction at the requested nod position.

$L 1521 F$-IRS. Only LL was observed for this object.

$L k C a$ 19. For this object, only the LH module was used, and it was pointed at the slit center instead of at the standard nod 1 and nod 2 positions. One-third of the data set consists of sky observations; this allowed accurate background subtraction, and therefore the signal from $\mathrm{LkCa} 19$, despite being faint ( $\sim 10 \mathrm{mJy})$, is reliable. Also, the IRS flux matches the MIPS $24 \mu \mathrm{m}$ (Luhman et al. 2010) quite well. 
$R X J 0432.8+1735$. The SL2 spectrum had to be scaled by a factor of 1.3 to match the flux level of SL1; this is most likely due to a small mispointing during the SL2 observation.

V410 X-ray 2. Only SH was used for this object. Also, the second observation of nod 2 was not usable, so it was left out from the average spectrum. Since no sky observation was obtained for this object, its spectrum also contains background emission, which should amount to about $40 \%$ at the shorter wavelengths and $20 \%$ at the longer wavelengths.

\section{REFERENCES}

Adame, L. 2010, PhD thesis, Univ. Nacional Autónoma de México

Adame, L., et al. 2011, ApJ, 726, L3

Alexander, R. D., \& Armitage, P. J. 2007, MNRAS, 375, 500

Alexander, R. D., Clarke, C. J., \& Pringle, J. E. 2006, MNRAS, 369, 229

Allers, K. N., Kessler-Silacci, J. E., Cieza, L. A., \& Jaffe, D. T. 2006, ApJ, 644, 364

André, P., Motte, F., \& Bacmann, A. 1999, ApJ, 513, L57

Andrews, S. M., Wilner, D. J., Espaillat, C., Hughes, A. M., Dullemond, C. P., McClure, M. K., Qi, C., \& Brown, J. M. 2011, ApJ, 732, 42

Apai, D., Pascucci, I., Bouwman, J., Natta, A., Henning, Th., \& Dullemond, C. P. 2005, Science, 310, 834

Artymowicz, P., \& Lubow, S. H. 1994, ApJ, 421, 651

Baraffe, I., Chabrier, G., Allard, F., \& Hauschildt, P. H. 2002, A\&A, 382, 563

Bary, J. S., Leisenring, J. M., \& Skrutskie, M. F. 2009, ApJ, 706, L168

Bertout, C. 1989, ARA\&A, 27, 351

Bertout, C., Robichon, N., \& Arenou, F. 1999, A\&A, 352, 574

Birnstiel, T., Ormel, C. W., \& Dullemond, C. P. 2011, A\&A, 525, A11

Boss, A. P. 2000, ApJ, 536, L101

Bourke, T. L., et al. 2006, ApJ, 649, L37

Bouwman, J., Lawson, W. A., Dominik, C., Feigelson, E. D., Henning, Th., Tielens, A. G. G. M., \& Waters, L. B. F. M. 2006, ApJ, 653, L57

Bouwman, J., et al. 2008, ApJ, 683, 479

Brauer, F., Dullemond, C. P., \& Henning, Th. 2008, A\&A, 480, 859

Briceño, C., Luhman, K. L., Hartmann, L., Stauffer, J. R., \& Kirkpatrick, J. D. 2002, ApJ, 580, 317

Brown, J. M., Blake, G. A., Qi, C., Dullemond, C. P., \& Wilner, D. J. 2008, ApJ, 675, L109

Brown, J. M., Blake, G. A., Qi, C., Dullemond, C. P., Wilner, D. J., \& Williams, J. P. 2009, ApJ, 704, 496

Brown, J. M., et al. 2007, ApJ, 664, L107

Burrows, C. J., et al. 1996, ApJ, 473, 437

Calvet, N., \& Gullbring, E. 1998, ApJ, 509, 802

Calvet, N., Muzerolle, J., Briceño, C., Hernández, J., Hartmann, L., Saucedo, J. L., \& Gordon, K. D. 2004, AJ, 128, 1294

Calvet, N., et al. 2005, ApJ, 630, L185

Chambers, J. E. 2006, ApJ, 652, L133

Ciesla, F. J. 2007, ApJ, 654, L159

Cieza, L. A., Kessler-Silacci, J. E., Jaffe, D. T., Harvey, P. M., \& Evans, N. J., II. 2005, ApJ, 635, 422

Clarke, C. J., Gendrin, A., \& Sotomayor, M. 2001, MNRAS, 328, 485

Cohen, M., Megeath, S. T., Hammersley, P. L., Martín-Luis, F., \& Stauffer, J. 2003, AJ, 125, 2645

Currie, T., Lada, C. J., Plavchan, P., Robitaille, T. P., Irwin, J., \& Kenyon, S. J. 2009, ApJ, 698, 1

D’Alessio, P., Calvet, N., Hartmann, L., Franco-Hernández, R., \& Servín, H. 2006, ApJ, 638, 314

D’Alessio, P., Calvet, N., Hartmann, L., Lizano, S., \& Cantó, J. 1999, ApJ, 527, 893

D’Alessio, P., Cantó, J., Calvet, N., \& Lizano, S. 1998, ApJ, 500, 411

D'Alessio, P., et al. 2005, ApJ, 621, 461

DeWarf, L. E., Sepinsky, J. F., Guinan, E. F., Ribas, I., \& Nadalin, I. 2003, ApJ, 590,357

de Zeeuw, P. T., Hoogerwerf, R., de Bruijne, J. H. J., Brown, A. G. A., \& Blaauw, A. 1999, AJ, 117, 354

Dorschner, J., Begemann, B., Henning, Th., Jäger, C., \& Mutschke, H. 1995, A\&A, 300, 503

Duchêne, G. 1999, A\&A, 341, 547

Duchêne, G., Bouvier, J., Bontemps, S., André, P., \& Motte, F. 2004, A\&A, 427, 651

Duchêne, G., Ghez, A. M., McCabe, C., \& Weinberger, A. J. 2003, ApJ, 592, 288

Duchêne, G., Monin, J.-L., Bouvier, J., \& Ménard, F. 1999, A\&A, 351, 954

Dullemond, C. P., \& Dominik, C. 2008, A\&A, 487, 205
Dullemond, C. P., Dominik, C., \& Natta, A. 2001, ApJ, 560, 957

Dullemond, C. P., van Zadelhoff, G. J., \& Natta, A. 2002, A\&A, 389, 464

Dunham, M. M., et al. 2006, ApJ, 651, 945

Dutrey, A., et al. 2008, A\&A, 490, L15

Eiroa, C., et al. 2002, A\&A, 384, 1038

Ercolano, B., Clarke, C. J., \& Robitaille, T. P. 2009, MNRAS, 394, L141

Ercolano, B., \& Owen, J. E. 2010, MNRAS, 406, 1553

Espaillat, C. 2009, PhD thesis, Univ. Michigan

Espaillat, C., Calvet, N., D’Alessio, P., Hernández, J., Qi, C., Hartmann, L., Furlan, E., \& Watson, D. M. 2007a, ApJ, 670, L135

Espaillat, C., Calvet, N., Luhman, K. L., Muzerolle, J., \& D'Alessio, P. 2008, ApJ, 682, L125

Espaillat, C., Furlan, E., D’Alessio, P., Sargent, B., Nagel, E., Calvet, N., Watson, D. M., \& Muzerolle, J. 2011, ApJ, 728, 49

Espaillat, C., et al. 2007b, ApJ, 664, L111

Espaillat, C., et al. 2010, ApJ, 717, 441

Fabian, D., Jäger, C., Henning, Th., Dorschner, J., \& Mutschke, H. 2000, A\&A, 364,282

Fazio, G. G., et al. 2004, ApJS, 154, 10

Feigelson, E. D., Welty, A. D., Imhoff, C. L., Hall, J. C., Etzel, P. B., Phillips, R. B., \& Lonsdale, C. J. 1994, ApJ, 432, 373

Fischer, W., Edwards, S., Hillenbrand, L., \& Kwan, J. 2011, ApJ, 730, 73

Forrest, W. J., et al. 2004, ApJS, 154, 443

Furlan, E., Forrest, W. J., Sargent, B. A., Manoj, P., Kim, K. H., \& Watson, D. M. 2009a, ApJ, 706, 1194

Furlan, E., et al. 2005, ApJ, 628, L65

Furlan, E., et al. 2006, ApJS, 165, 568

Furlan, E., et al. 2008, ApJS, 176, 184

Furlan, E., et al. 2009b, ApJ, 703, 1964

Gail, H.-P. 2004, A\&A, 413, 571

Ghez, A. M., Neugebauer, G., Gorham, P. W., Haniff, C. A., Kulkarni, S. R., Matthews, K., Koresko, Ch., \& Beckwith, S. 1991, AJ, 102, 2066

Ghez, A. M., Neugebauer, G., \& Matthews, K. 1993, AJ, 106, 2005

Gomez, M., Kenyon, S. J., \& Hartmann, L. 1994, AJ, 107, 1850

Guenther, E. W., \& Ball, M. 1999, A\&A, 347, 508

Guieu, S., Dougados, C., Monin, J.-L., Magnier, E., \& Martín, E. L. 2006, A\&A, 446,485

Guilloteau, S., Dutrey, A., Pety, J., \& Gueth, F. 2008, A\&A, 478, L31

Gullbring, E., Calvet, N., Muzerolle, J., \& Hartmann, L. 2000, ApJ, 544, 927

Harker, D. E., \& Desch, S. J. 2002, ApJ, 565, L109

Hartigan, P., Hartmann, L., Kenyon, S., Hewett, R., \& Stauffer, J. 1989, ApJS, 70,899

Hartigan, P., \& Kenyon, S. J. 2003, ApJ, 583, 334

Hartigan, P., Kenyon, S. J., Hartmann, L., Strom, S. E., Edwards, S., Welty, A. D., \& Stauffer, J. 1991, ApJ, 382, 617

Hartigan, P., Strom, K. M., \& Strom, S. E. 1994, ApJ, 427, 961

Hartmann, L., Hewett, R., \& Calvet, N. 1994, ApJ, 426, 669

Hartmann, L., Megeath, S. T., Allen, L., Luhman, K., Calvet, N., D’Alessio, P., Franco-Hernández, R., \& Fazio, G. 2005, ApJ, 629, 881

Herbig, G. H., \& Bell, K. R. 1988, Lick Observatory Bulletin (Santa Cruz, CA: Lick Observatory)

Herczeg, G. J., \& Hillenbrand, L. A. 2008, ApJ, 681, 594

Higdon, S. J. U., et al. 2004, PASP, 116, 975

Houck, J. R., et al. 2004, ApJS, 154, 18

Hughes, A. M., et al. 2009, ApJ, 698, 131

Ida, S., \& Lin, D. N. C. 2010, ApJ, 719, 810

Ireland, M. J., \& Kraus, A. L. 2008, ApJ, 678, L59

Isella, A., Carpenter, J. M., \& Sargent, A. I. 2010, ApJ, 714, 1746

Itoh, Y., Tamura, M., \& Nakajima, T. 1999, AJ, 117, 1471

Itoh, Y., Tamura, M., \& Tokunaga, A. 2002, PASJ, 54, 561

Jäger, C., Molster, F. J., Dorschner, J., Henning, Th., Mutschke, H., \& Waters, L. B. F. M. 1998, A\&A, 339, 904

Jayawardhana, R., Mohanty, S., \& Basri, G. 2003, ApJ, 592, 282

Jensen, E. L. N., \& Mathieu, R. D. 1997, AJ, 114, 301

Jensen, E. L. N., Mathieu, R. D., \& Fuller, G. A. 1994, ApJ, 429, L29

Johansen, A., Brauer, F., Dullemond, C., Klahr, H., \& Henning, Th. 2008, A\&A, 486, 597

Juhász, A., et al. 2010, ApJ, 721, 431

Kenyon, S. J., Brown, D. I., Tout, Ch. A., \& Berlind, P. 1998, AJ, 115, 2491

Kenyon, S. J., Gomez, M., Marzke, R. O., \& Hartmann, L. 1994, AJ, 108, 251

Kenyon, S. J., Gómez, M., \& Whitney, B. A. 2008, in Handbook of Star Forming Regions, Vol. 1: The Northern Sky, ed. B. Reipurth (ASP Monograph Publ. Vol. 4; San Francisco, CA: ASP), 405

Kenyon, S. J., \& Hartmann, L. 1987, ApJ, 323, 714

Kenyon, S. J., \& Hartmann, L. 1995, ApJS, 101, 117

Kessler-Silacci, J., et al. 2006, ApJ, 639, 275

Kessler-Silacci, J. E., et al. 2007, ApJ, 659, 680 
Kim, K. H., et al. 2009, ApJ, 700, 1017

Konopacky, Q. M., Ghez, A. M., Rice, E. L., \& Duchêne, G. 2007, ApJ, 663, 394

Koresko, Ch. D. 2000, ApJ, 531, L147

Kraus, A. L., \& Hillenbrand, L. A. 2007, ApJ, 662, 413

Kraus, A. L., \& Hillenbrand, L. A. 2009, ApJ, 703, 1511

Kraus, A. L., Ireland, M. J., Martinache, F., \& Hillenbrand, L. A. 2011, ApJ, 731,8

Krist, J. E., et al. 1998, ApJ, 501, 841

Lada, C. J. 1987, in IAU Symp. 115, Star Forming Regions, ed. M. Peimbert \& J. Jugaku (Dordrecht: Reidel), 1

Lada, C. J., \& Wilking, B. A. 1984, ApJ, 287, 610

Lada, C. J., et al. 2006, AJ, 131, 1574

Lebouteiller, V., Bernard-Salas, J., Sloan, G. C., \& Barry, D. J. 2010, PASP, 122,231

Leinert, Ch., Richichi, A., \& Haas, M. 1997, A\&A, 318, 472

Leinert, Ch., Zinnecker, H., Weitzel, N., Christou, J., Ridgway, S. T., Jameson, R., Haas, M., \& Lenzen, R. 1993, A\&A, 278, 129

Luhman, K. L. 2000, ApJ, 544, 1044

Luhman, K. L. 2004, ApJ, 617, 1216

Luhman, K. L. 2006, ApJ, 645, 676

Luhman, K. L., Allen, P. R., Espaillat, C., Hartmann, L., \& Calvet, N. 2010, ApJS, 186, 111

Luhman, K. L., Mamajek, E. E., Allen, P. R., \& Cruz, K. L. 2009, ApJ, 703, 399

Luhman, K. L., et al. 2007, ApJ, 666, 1219

Luhman, K. L., et al. 2008, ApJ, 675, 1375

Manoj, P., et al. 2011, ApJS, 193, 11

Marsh, K. A., \& Mahoney, M. J. 1992, ApJ, 395, L115

Martín, E. L., Basri, G., \& Zapatero Osorio, M. R. 1999, AJ, 118, 1005

Mathieu, R. D., Stassun, K., Basri, G., Jensen, E. L. N., Johns-Krull, Ch. M., Valenti, J. A., \& Hartmann, L. W. 1997, AJ, 113, 1841

Mathis, J. S. 1990, ARA\&A, 28, 37

McCabe, C., Ghez, A. M., Prato, L., Duchêne, G., Fisher, R. S., \& Telesco, C. 2006, ApJ, 636, 932

McClure, M. K. 2009, ApJ, 693, L81

McClure, M. K., et al. 2008, ApJ, 683, L187

McClure, M. K., et al. 2010, ApJS, 188, 75

Meeus, G., Sterzik, M., Bouwman, J., \& Natta, A. 2003, A\&A, 409, L25

Meeus, G., Waters, L. B. F. M., Bouwman, J., van den Ancker, M. E., Waelkens, C., \& Malfait, K. 2001, A\&A, 365, 476

Merín, B., et al. 2010, ApJ, 718, 1200

Meru, F., \& Bate, M. R. 2011, MNRAS, 410, 559

Meyer, M. R., Calvet, N., \& Hillenbrand, L. A. 1997, AJ, 114, 288

Miyake, K., \& Nakagawa, Y. 1995, ApJ, 441, 361

Mohanty, S., Jayawardhana, R., \& Basri, G. 2005, ApJ, 626, 498

Mohanty, S., Jayawardhana, R., Natta, A., Fujiyoshi, T., Tamura, M., \& Barrado y Navascués, D. 2004, ApJ, 609, L33

Monin, J.-L., Ménard, F., \& Duchêne, G. 1998, A\&A, 339, 113

Monnier, J. D., Tannirkulam, A., Tuthill, P. G., Ireland, M., Cohen, R., Danchi, W. C., \& Baron, F. 2008, ApJ, 681, L97

Moriarty-Schieven, G. H., Wannier, P. G., Tamura, M., \& Keene, J. 1992, ApJ, 400, 260

Movshovitz, N., Bodenheimer, P., Podolak, M., \& Lissauer, J. J. 2010, Icarus, 209, 616

Muzerolle, J., Allen, L. E., Megeath, S. T., Hernández, J., \& Gutermuth, R. A. 2010, ApJ, 708, 1107

Muzerolle, J., Hillenbrand, L., Calvet, N., Briceño, C., \& Hartmann, L. 2003, ApJ, 592, 266

Muzerolle, J., Luhman, K. L., Briceño, C., Hartmann, L., \& Calvet, N. 2005, ApJ, 625, 906

Muzerolle, J., et al. 2009, ApJ, 704, L15

Nagel, E., D’Alessio, P., Calvet, N., Espaillat, C., Sargent, B., Hernández, J., \& Forrest, W. J. 2010, ApJ, 708, 38

Natta, A., Testi, L., Comerón, F., Oliva, E., D’Antona, F., Baffa, C., Comoretto, G., \& Gennari, S. 2002, A\&A, 393, 597

Natta, A., Testi, L., Muzerolle, J., Randich, S., Comerón, F., \& Persi, P. 2004, A\&A, 424, 603

Nguyen, D. C., Jayawardhana, R., van Kerkwijk, M. H., Brandeker, A., Scholz, A., \& Damjanov, I. 2009, ApJ, 695, 1648

Olofsson, J., et al. 2009, A\&A, 507, 327

Owen, J. E., Ercolano, B., Clarke, C. J., \& Alexander, R. D. 2010, MNRAS, 401, 1415

Padgett, D. L., et al. 2006, ApJ, 645, 1283

Pascucci, I., Apai, D., Hardegree-Ullman, E. E., Kim, J. S., Meyer, M. R., \& Bouwman, J. 2008, ApJ, 673, 477
Pascucci, I., Apai, D., Luhman, K., Henning, Th., Bouwman, J., Meyer, M. R., Lahuis, F., \& Natta, A. 2009, ApJ, 696, 143

Piétu, V., Dutrey, A., Guilloteau, S., Chapillon, E., \& Pety, J. 2006, A\&A, 460, L43

Pollack, J. B., Hubickyj, O., Bodenheimer, P., Lissauer, J. J., Podolak, M., \& Greenzweig, Y. 1996, Icarus, 124, 62

Pott, J.-U., Perrin, M. D., Furlan, E., Ghez, A. M., Herbst, T. M., \& Metchev, S. 2010, ApJ, 710, 265

Prato, L., \& Simon, M. 1997, ApJ, 474, 455

Press, W. H., Teukolsky, S. A., Vetterling, W. T., \& Flannery, B. P. 1993, Numerical Recipes in C (Cambridge: Cambridge Univ. Press)

Prusti, T., Clark, F. O., Laureijs, R. J., Wakker, B. P., \& Wesselius, P. R. 1992 A\&A, 259, 537

Ratzka, Th., et al. 2009, A\&A, 502, 623

Rayner, J. T., Toomey, D. W., Onaka, P. M., Denault, A. J., Stahlberger, W. E., Vacca, W. D., \& Cushing, M. C. 2003, PASP, 115, 362

Rebull, L. M., et al. 2010, ApJS, 186, 259

Riaz, B. 2009, ApJ, 701, 571

Richichi, A., Leinert, Ch., Jameson, R., \& Zinnecker, H. 1994, A\&A, 287, 145

Rieke, G. H., et al. 2004, ApJS, 154, 25

Robitaille, T. P., Whitney, B. A., Indebetouw, R., Wood, K., \& Denzmore, P. 2006, ApJS, 167, 256

Salyk, C., Blake, G. A., Boogert, A. C. A., \& Brown, J. M. 2009, ApJ, 699, 330

Sargent, B., et al. 2006, ApJ, 645, 395

Sargent, B. A., et al. 2009, ApJS, 182, 477

Schaefer, G. H., Dutrey, A., Guilloteau, S., Simon, M., \& White, R. J. 2009, ApJ, 701,698

Schaefer, G. H., Simon, M., Beck, T. L., Nelan, E., \& Prato, L. 2006, AJ, 132 2618

Schaefer, G. H., Simon, M., Nelan, E., \& Holfeltz, S. T. 2003, AJ, 126, 1971

Shuping, R. Y., Chiar, J. E., Snow, T. P., \& Kerr, T. 2001, ApJ, 547, L161

Sicilia-Aguilar, A., Hartmann, L. W., Watson, D., Bohac, C., Henning, Th., \& Bouwman, J. 2007, ApJ, 659, 1637

Sicilia-Aguilar, A., Henning, Th., Juhász, A., Bouwman, J., Garmire, G., \& Garmire, A. 2008, ApJ, 687, 1145

Simon, M., Chen, W. P., Howell, R. R., Benson, J. A., \& Slowik, D. 1992, ApJ, 384,212

Simon, M., et al. 1995, ApJ, 443, 625

Skemer, A. J., Close, L. M., Hinz, P. M., Hoffmann, W. F., Kenworthy, M. A., \& Miller, D. L. 2008, ApJ, 676, 1082

Skrutskie, M. F., Dutkevitch, D., Strom, S. E., Edwards, S., Strom, K. M., \& Shure, M. A. 1990, AJ, 99, 1187

Skrutskie, M. F., et al. 2006, AJ, 131, 1163

Smith, K. W., Balega, Y. Y., Duschl, W. J., Hofmann, K.-H., Lachaume, R., Preibisch, T., Schertl, D., \& Weigelt, G. 2005, A\&A, 431, 307

Strom, K. M., \& Strom, S. E. 1994, ApJ, 424, 237

Strom, K. M., Strom, S. E., Edwards, S., Cabrit, S., \& Skrutskie, M. F. 1989, AJ, 97, 1451

Szücs, L., Apai, D., Pascucci, I., \& Dullemond, C. 2010, ApJ, 720, 1668

Takeuchi, T., Miyama, S. M., \& Lin, D. N. C. 1996, ApJ, 460, 832

Tamura, M., Gatley, I., Waller, W., \& Werner, M. W. 1991, ApJ, 374, L25

Thalmann, C., et al. 2010, ApJ, 718, L87

Todorov, K., Luhman, K. L., \& McLeod, K. K. 2010, ApJ, 714, L84

Turner, N. J., Carballido, A., \& Sano, T. 2010, ApJ, 708, 188

van Boekel, R., Min, M., Waters, L. B. F. M., de Koter, A., Dominik, C., van den Ancker, M. E., \& Bouwman, J. 2005, A\&A, 437, 189

van Boekel, R., Waters, L. B. F. M., Dominik, C., Bouwman, J., de Koter, A., Dullemond, C. P., \& Paresce, F. 2003, A\&A, 400, L21

Ward, W. R. 1997, Icarus, 126, 261

Watson, A. M., \& Stapelfeldt, K. R. 2004, ApJ, 602, 860

Watson, D. M., et al. 2009, ApJS, 180, 84

Weidenschilling, S. J. 1997, Icarus, 127, 290

Werner, M. W., et al. 2004, ApJS, 154, 1

White, R. J., \& Basri, G. 2003, ApJ, 582, 1109

White, R. J., \& Ghez, A. M. 2001, ApJ, 556, 265

White, R. J., Ghez, A. M., Reid, I. N., \& Schultz, G. 1999, ApJ, 520, 811

White, R. J., \& Hillenbrand, L. A. 2004, ApJ, 616, 998

Wichmann, R., et al. 1996, A\&A, 312, 439

Winston, E., et al. 2009, AJ, 137, 4777

Wooden, D. H., Bell, K. R., Harker, D. E., \& Woodward, C. E. 2000, BAAS, 32, 1482

Young, C. H., Shirley, Y. L., Evans, N. J., II, \& Rawlings, J. M. C. 2003, ApJS, 145,111

Zhu, Z., Nelson, R. P., Hartmann, L., Espaillat, C., \& Calvet, N. 2011, ApJ, 729, 47 